\title{
Splittings and automorphisms of relatively hyperbolic groups
}

\author{
Vincent Guirardel and Gilbert Levitt ${ }^{1}$
}

\begin{abstract}
We study automorphisms of a relatively hyperbolic group $G$. When $G$ is oneended, we describe $\operatorname{Out}(G)$ using a preferred JSJ tree over subgroups that are virtually cyclic or parabolic. In particular, when $G$ is toral relatively hyperbolic, $\operatorname{Out}(G)$ is virtually built out of mapping class groups and subgroups of $\mathrm{GL}_{n}(\mathbb{Z})$ fixing certain basis elements. When more general parabolic groups are allowed, these subgroups of $\mathrm{GL}_{n}(\mathbb{Z})$ have to be replaced by McCool groups: automorphisms of parabolic groups acting trivially (i.e. by conjugation) on certain subgroups.

Given a malnormal quasiconvex subgroup $P$ of a hyperbolic group $G$, we view $G$ as hyperbolic relative to $P$ and we apply the previous analysis to describe the $\operatorname{group} \operatorname{Out}(P \uparrow G)$ of automorphisms of $P$ that extend to $G$ : it is virtually a McCool group. If $\operatorname{Out}(P \uparrow G)$ is infinite, then $P$ is a vertex group in a splitting of $G$. If $P$ is torsion-free, then $\operatorname{Out}(P \uparrow G)$ is of type VF, in particular finitely presented.

We also determine when $\operatorname{Out}(G)$ is infinite, for $G$ relatively hyperbolic. The interesting case is when $G$ is infinitely-ended and has torsion. When $G$ is hyperbolic, we show that $\operatorname{Out}(G)$ is infinite if and only if $G$ splits over a maximal virtually cyclic subgroup with infinite center. In general we show that infiniteness of $\operatorname{Out}(G)$ comes from the existence of a splitting with infinitely many twists, or having a vertex group that is maximal parabolic with infinitely many automorphisms acting trivially on incident edge groups.
\end{abstract}

Mathematics Subject Classification (2010). 20F28, 20F65, 20F67, 20F34, 20E08, 57M07.

Keywords. Groups of automorphisms, relatively hyperbolic group, splitting, hyperbolic group, groups acting on trees.

${ }^{1}$ This work was partly supported by ANR-07-BLAN-0141, ANR-2010-BLAN-116-01, ANR06-JCJC-0099-01, ANR-11-BS01-013-01. 


\section{Contents}

1 Introduction . . . . . . . . . . . . . . . . . . . . 600

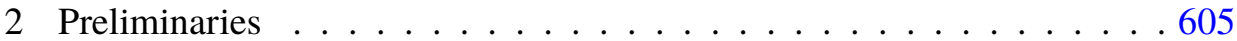

3 Relatively hyperbolic groups . . . . . . . . . . . . . . . 615

4 Automorphisms of one-ended relatively hyperbolic groups . . . . . . . 620

5 The modular group . . . . . . . . . . . . . . . . . . .628

6 Induced automorphisms . . . . . . . . . . . . . . . . .634

7 Groups with infinitely many automorphisms . . . . . . . . . 638

8 Fixed subgroups . . . . . . . . . . . . . . . . . . . . . . . . . . . . 649

9 Rigid groups have finitely many automorphisms . . . . . . . . . 651

References . . . . . . . . . . . . . . . . . . . . . .659

\section{Introduction}

This paper studies automorphisms of hyperbolic and relatively hyperbolic groups in relation with their splittings. The first result in this direction is due to Paulin [54]: if $G$ is a hyperbolic group with $\operatorname{Out}(G)$ infinite, then $G$ has an action on an $\mathbb{R}$-tree with virtually cyclic (possibly finite) arc stabilizers. Rips theory then implies that $G$ splits over a virtually cyclic group.

Understanding the global structure of $\operatorname{Out}(G)$ requires techniques which depend on the number of ends of $G$. If $G$ is one-ended, there is an $\operatorname{Out}(G)$-invariant JSJ decomposition, and its study leads to Sela's description of $\operatorname{Out}(G)$ as a virtual extension of a direct product of mapping class groups by a virtually abelian group $[59,45]$. If $G$ has infinitely many ends, one does not get such a precise description because there is no $\operatorname{Out}(G)$-invariant splitting. One may study $\operatorname{Out}(G)$ by letting it act on a suitable space of splittings, the most famous being Culler-Vogtmann's outer space for $\operatorname{Out}\left(F_{n}\right)$.

Before moving on to relatively hyperbolic groups, here is a basic problem about which we get new results in the context of hyperbolic, and even free, groups. Given a finitely generated subgroup $P$ of a group $G$, consider the group $\operatorname{Out}(P \uparrow G) \subset$ Out $(P)$ consisting of outer automorphisms of $P$ which extend to automorphisms of $G$. What can one say about this group ? For instance, is it finitely generated? finitely presented? This question was asked by D. Calegari for automorphisms of free groups, and we answer it when $P$ is malnormal.

The answer is related to splittings through the following simple remark: if $P$ is a vertex group in a splitting of $G$, say $G=A *_{C_{1}} P *_{C_{2}} B$, then any automorphism of $P$ which acts trivially (i.e. as conjugation by some element $p_{i} \in P$ ) on each 
incident edge group $C_{i}$, extends to $G$ (this is the algebraic translation of the fact that any self-homeomorphism of a closed subset $Y \subset X$ which is the identity on the frontier of $Y$ extends by the identity to a homeomorphism of $X$ ).

The following theorem says that this phenomenon accounts for almost all of $\operatorname{Out}(P \uparrow G)$.

Theorem 1.1 (see Corollary 6.3). Let $P$ be a quasiconvex malnormal subgroup of a hyperbolic group $G$. If $\operatorname{Out}(P \uparrow G)$ is infinite, then $P$ is a vertex group in a splitting of $G$, and the group of outer automorphisms of $P$ acting trivially on incident edge groups has finite index in $\operatorname{Out}(P \uparrow G)$.

We call the group of outer automorphisms of $P$ acting trivially on a family of subgroups a $\mathrm{McC}$ Cool group of $P$, because of McCool's paper about subgroups of $\operatorname{Out}\left(F_{n}\right)$ fixing a finite set of conjugacy classes [50]. In this language, Theorem 1.1 says that $\operatorname{Out}(P \uparrow G)$ is virtually a McCool group of $P$. It is a theme of this paper that many groups of automorphisms may be understood in terms of McCool groups, and that many results concerning the full group $\operatorname{Out}(G)$ also apply to McCool groups (see also [37]).

The groups considered by McCool are finitely presented [50]. In fact, they have a finite index subgroup with a finite classifying space [13]. In [37] we extend these results to all McCool groups of torsion-free hyperbolic groups (and more generally of toral relatively hyperbolic groups). From this, one deduces that $\operatorname{Out}(P \uparrow G)$ has a finite index subgroup with a finite classifying space when $G$ and $P$ are as in Theorem 1.1, with $G$ torsion-free.

Our hypotheses for Theorem 1.1, namely quasiconvexity and malnormality of $P$, imply that $G$ is hyperbolic relative to $P$ (see [6]). In fact, Theorem 1.1 is just a special case of a result describing $\operatorname{Out}(P \uparrow G)$ as a virtual $\mathrm{McCool}$ group when $G$ is relatively hyperbolic and $P$ is a maximal parabolic subgroup (see Theorem 6.2 for a precise statement).

This paper also addresses the question of whether $\operatorname{Out}(G)$ is finite or infinite. It turns out that the answer is much simpler when $G$ is torsion-free, owing to the fact that $\operatorname{Out}(G)$ is then infinite whenever $G$ has infinitely many ends (see Lemma 7.1).

Things are more complicated when torsion is allowed. For instance, characterizing virtually free groups with $\operatorname{Out}(G)$ infinite is a non-trivial problem which was solved by Pettet [56]. The following theorem gives a different characterization. We say that a subgroup of $G$ is $z_{\max }$ if it is maximal for inclusion among virtually cyclic subgroups with infinite center. 
Theorem 1.2 (see Theorems 7.14 and 7.15). Let $G$ be a hyperbolic group. Then $\operatorname{Out}(G)$ is infinite if and only $G$ splits over a $Z_{\max }$ subgroup $C$; in this case, any element $c \in C$ of infinite order defines a Dehn twist which has infinite order in $\operatorname{Out}(G)$.

Moreover, one may decide algorithmically whether $\operatorname{Out}(G)$ is finite or infinite.

The first assertion answers a question asked by D. Groves. See [10] for a related result proved independently by $\mathrm{M}$. Carette, and $[45,17]$ for the one-ended case.

Let us now consider (in)finiteness of $\operatorname{Out}(G)$ when $G$ is relatively hyperbolic. Suppose that $G$ is hyperbolic with respect to a finite family $\mathcal{P}$ of finitely generated subgroups $P_{i}$. Since automorphisms of $G$ need not respect $\mathcal{P}$ (for instance, $G$ may be free and $\mathcal{P}$ may consist of any finitely generated malnormal subgroup), we consider the group $\operatorname{Out}(G ; \mathcal{P})$ consisting of automorphisms mapping each $P_{i}$ to a conjugate (in an arbitrary way). Note that $\operatorname{Out}(G ; \mathcal{P})$ has finite index in the full group $\operatorname{Out}(G)$ when the groups $P_{i}$ are small but not virtually cyclic, more generally when they are not themselves relatively hyperbolic in a nontrivial way [51].

Given a splitting of $G$, we have already pointed out that any automorphism of a vertex group acting trivially on incident edge groups extends to an automorphism of $G$. Twists around edges of the splitting also provide automorphisms of $G$. For instance, if $G=A *_{C} B$, and $a \in A$ centralizes $C$, there is an automorphism of $G$ equal to conjugation by $a$ on $A$ and to the identity on $B$. Note that we do not require that $C$ be virtually cyclic or that $a \in C$ (see Subsection 2.6).

The following result says that infiniteness of $\operatorname{Out}(G ; \mathcal{P})$ comes from twists or from a McCool group of a parabolic group.

Theorem 1.3 (see Corollary 7.13). Let $G$ be hyperbolic relative to a family $\mathcal{P}=$ $\left\{P_{1}, \ldots, P_{n}\right\}$ of finitely generated subgroups. Then $\operatorname{Out}(G ; \mathcal{P})$ is infinite if and only if $G$ has a splitting over virtually cyclic or parabolic subgroups, with each $P_{i}$ contained in a conjugate of a vertex group, such that one of the following holds:

- the group of twists of the splitting is infinite;

- some $P_{i}$ is a vertex group and there are infinitely many outer automorphisms of $P_{i}$ acting trivially on incident edge groups.

As mentioned above, one can get similar results characterizing the infiniteness of McCool groups of $G$. We refer to Section 7, in particular Theorem 7.6 and Corollary 7.13 , for more detailed statements.

Let us now discuss the techniques that we use. We assume that $G$ is hyperbolic relative to $\mathcal{P}$, and we distinguish two cases according to the number of ends 
(technically, we consider relative one-endedness, but we will ignore this in the introduction).

When $G$ is one-ended, we use a canonical $\operatorname{Out}(G ; \mathcal{P})$-invariant decomposition $\Gamma_{\text {can }}$ of $G$, namely (see Subsection 3.3) the JSJ decomposition over elementary (i.e. parabolic or virtually cyclic) subgroups relative to parabolic subgroups (i.e. parabolic subgroups have to be contained in conjugates of vertex groups).

One may thus generalize the description of $\operatorname{Out}(G)$ given by Sela for $G$ hyperbolic, and express $\operatorname{Out}(G ; \mathcal{P})$ in terms of mapping class groups, McCool groups of maximal parabolic subgroups, and a group of twists $\mathcal{T}$. For simplicity we restrict to a special case here (see Section 4 for a general statement).

Theorem 1.4 (see Corollary 4.4). Let $G$ be toral relatively hyperbolic and oneended. Then some finite index subgroup $\operatorname{Out}^{1}(G)$ of $\operatorname{Out}(G)$ fits in an exact sequence

$$
1 \longrightarrow \mathcal{T} \longrightarrow \operatorname{Out}^{1}(G) \longrightarrow \prod_{i=1}^{p} M C G^{0}\left(\Sigma_{i}\right) \times \prod_{k=1}^{m} \mathrm{GL}_{r_{k}, n_{k}}(\mathbb{Z}) \longrightarrow 1
$$

where $\mathcal{T}$ is finitely generated free abelian, $\operatorname{MCG}^{0}\left(\Sigma_{i}\right)$ is the group of isotopy classes of homeomorphisms of a compact surface $\Sigma_{i}$ mapping each boundary component to itself in an orientation-preserving way, and $\mathrm{GL}_{r, n}(\mathbb{Z})$ is the group of automorphisms of $\mathbb{Z}^{r+n}$ fixing the first $n$ generators.

More generally, McCool groups of a one-ended toral relatively hyperbolic group $G$ have a similar description (see Corollary 4.9). A more general statement (without restriction on the parabolic subgroups) is given in Theorems 4.3 and 4.6.

We also show that the modular group of $G$, introduced by Sela $[60,61]$ and usually defined by considering all suitable splittings of $G$, may be seen on the single splitting $\Gamma_{\text {can }}$. We refer to Section 5 for details.

To prove Theorem 1.1 when $G$ is one-ended, one applies the previous analysis, viewing $G$ as hyperbolic relative to $P$. Note that we use a JSJ decomposition which is relative (to $P$ ), and over subgroups which are not small (any subgroup of $P$ is allowed).

Another example of the usefulness of relative JSJ decompositions is to prove the Scott conjecture about fixed subgroups of automorphisms of free groups. The proof that we give in Section 8, though not really new, is simplified by the use of the cyclic JSJ decomposition relative to the fixed subgroup.

We therefore work consistently in a relative context. We fix another family of finitely generated subgroups $\mathcal{H}=\left\{H_{1}, \ldots, H_{q}\right\}$, and we define $\operatorname{Out}\left(G ; \mathcal{P}, \mathcal{H}^{(\mathrm{t})}\right)$ 
as the group of automorphisms mapping $P_{i}$ to a conjugate (in an arbitrary way) and acting trivially on $H_{j}$ (i.e. as conjugation by an element $g_{j}$ of $G$ ).

In order to understand the structure of the automorphism group of a one-ended relatively hyperbolic group from its canonical JSJ decomposition, one needs to control automorphisms of rigid vertex groups. This is made possible by the following generalisation of Paulin's theorem mentioned above:

Theorem 1.5 (see Theorem 3.9). Let $G$ be hyperbolic relative to $\mathcal{P}=\left\{P_{1}, \ldots, P_{n}\right\}$, with $P_{i}$ finitely generated and $P_{i} \neq G$. Let $\mathcal{H}=\left\{H_{1}, \ldots, H_{q}\right\}$ be another family of finitely generated subgroups.

If $\operatorname{Out}\left(G ; \mathcal{P}, \mathcal{H}^{(\mathrm{t})}\right)$ is infinite, then $G$ splits over an elementary (virtually cyclic or parabolic) subgroup relative to $\mathcal{P} \cup \mathcal{H}$.

Note that there is no quasiconvexity or malnormality assumption on groups in $\mathcal{H}$, but the automorphisms that we consider have to act trivially on them (see also Remark 9.3).

The theorem is proved using the Bestvina-Paulin method (extended to relatively hyperbolic groups in [1]) to get an action on an $\mathbb{R}$-tree $T$, and then applying Rips theory as developed in [3] to get a splitting. There are technical difficulties in the second step because $G$ may fail to be finitely presented (the $P_{i}$ 's are not required to be finitely presented), and the action on $T$ may fail to be stable if the $P_{i}$ 's are not slender; it only satisfies a weaker property which we call hypostability, and in the last section we generalize [3] to hypostable actions of relatively finitely presented groups.

Theorem 1.5 explains why McCool groups appear in Theorems 1.3 and 1.4. Indeed, given a rigid vertex group $G_{v}$ in a JSJ decomposition of a one-ended group, Theorem 1.5 implies that only finitely many outer automorphisms of $G_{v}$ extend to automorphisms of $G$. In turn, this implies that, after passing to a finite index subgroup, automorphisms of $G$ act trivially on edge groups of the JSJ decomposition. See Subsection 4.1 for details.

When $G$ is not one-ended, one has to consider splittings over finite groups. We do not have an exact sequence as in Theorem 1.4 because there is no $\operatorname{Out}(G ; \mathcal{P})$-invariant splitting. In order to prove Theorems 1.2 and 1.3 , we use the tree of cylinders introduced in [34] to obtain a non-trivial splitting over finite groups which is invariant or has an infinite group of twists (Corollary 7.11).

The paper is organized as follows. Section 2 consists of preliminaries (JSJ decompositions, automorphisms of a tree, trees of cylinders). Section 3 contains generalities about relatively hyperbolic groups. We point out that vertex groups of a splitting over relatively quasiconvex subgroups are relatively quasi- 
convex, and that the canonical JSJ decomposition $\Gamma_{\text {can }}$ has finitely generated edge groups. In Section 4 we study the structure of the automorphism group of a oneended relatively hyperbolic group. Section 5 is devoted to the modular group. In Section 6 we study extendable automorphisms; Theorem 1.1 is a special case of Theorem 6.2. Section 7 is devoted to the question of whether groups like $\operatorname{Out}\left(G ; \mathcal{P}, \mathcal{H}^{(\mathrm{t})}\right)$ and $\operatorname{Out}\left(G ; \mathcal{P}^{(\mathrm{t})}, \mathcal{H}^{(\mathrm{t})}\right)$ are finite or infinite. Section 8 contains a proof of the Scott conjecture, and a partial generalization to relatively hyperbolic groups. Theorem 1.5 is proved in Section 9.

Acknowledgments. We thank D. Groves and D. Calegari for asking stimulating questions, and the organizers of the 2007 Geometric Group Theory program at MSRI where this research was started. We also thank the referee for helpful comments.

\section{Preliminaries}

Unless mentioned otherwise, $G$ will always be a finitely generated group.

Given a group $A$ and a subgroup $B$, we denote by $Z(A)$ the center of $A$, by $Z_{A}(B)$ the centralizer of $B$ in $A$, and by $N_{A}(B)$ the normalizer of $B$ in $A$. We write $B^{g}$ for $g B g^{-1}$.

A subgroup $B \subset A$ is malnormal if $B^{g} \cap B$ is trivial for all $g \notin B$, almost malnormal if $B^{g} \cap B$ is finite for all $g \notin B$.

A group is virtually cyclic if it has a cyclic subgroup of finite index; it may be finite or infinite. Its outer automorphism group is finite.

A group $G$ is slender if $G$ and all its subgroups are finitely generated. We say that $G$ is small if it contains no non-abelian free group (see [2] for a slightly weaker definition).

Let $\mathcal{P}$ be a family of subgroups $P_{i}$. In most cases, $\mathcal{P}$ will be a finite collection of finitely generated groups $\mathcal{P}=\left\{P_{1}, \ldots, P_{n}\right\}$.

The group $G$ is finitely presented relative to $\mathcal{P}=\left\{P_{1}, \ldots, P_{n}\right\}$ if it is the quotient of $P_{1} * \cdots * P_{n} * F$ by the normal closure of a finite subset, with $F$ a finitely generated free group. If $G$ is finitely presented relative to $\mathcal{P}=\left\{P_{1}, \ldots, P_{n}\right\}$, and if $g_{1}, \ldots, g_{n} \in G$, then $G$ is also finitely presented relative to $\left\{P_{1}^{g_{1}}, \ldots, P_{n}^{g_{n}}\right\}$.

Let $H$ be a finitely generated subgroup. If $G$ is finitely presented relative to $\mathcal{P}$, then $G$ is finitely presented relative to $\mathcal{P} \cup\{H\}$; the converse is not true in general. 
2.1. Relative automorphisms. Given $G$ and $\mathcal{P}$, we denote by $\operatorname{Aut}(G ; \mathcal{P})$ the subgroup of $\operatorname{Aut}(G)$ consisting of automorphisms mapping each $P_{i}$ to a conjugate, and by $\operatorname{Out}(G ; \mathcal{P})$ its image in $\operatorname{Out}(G)$. If $\mathcal{P}=\left\{P_{1}, \ldots, P_{n}\right\}$, we use the notations $\operatorname{Aut}\left(G ; P_{1}, \ldots, P_{n}\right)$ and $\operatorname{Out}\left(G ; P_{1}, \ldots, P_{n}\right)$. We also write $\operatorname{Out}(G ; \mathcal{P}, Q)$ for $\operatorname{Out}(G ; \mathcal{P} \cup \mathcal{Q})$.

We define $\operatorname{Out}\left(G ; \mathcal{P}^{(\mathrm{t})}\right) \subset \operatorname{Out}(G ; \mathcal{P})$ by restricting to automorphisms whose restriction to each $P_{i}$ agrees with conjugation by some element $g_{i}$ of $G$ (we call them marked automorphisms, or automorphisms acting trivially on $\mathcal{P}$ ). An outer automorphism $\Phi$ belongs to $\operatorname{Out}\left(G ; \mathcal{P}^{(\mathrm{t})}\right)$ if and only if, for each $i$, it has a representative $\alpha_{i} \in \operatorname{Aut}(G)$ equal to the identity on $P_{i}$.

The group $\operatorname{Out}\left(G ; \mathcal{P}^{(\mathrm{t})}\right)$ is denoted by PMCG in [45], by $\operatorname{Out}_{m}(G ; \mathcal{P})$ in [17], and is called a (generalized) McCool group in [37].

Note that $\operatorname{Out}\left(G ; G^{(\mathrm{t})}\right)$ is trivial, and that $\operatorname{Out}\left(G ; \mathcal{P}^{(\mathrm{t})}\right)$ has finite index in $\operatorname{Out}(G ; \mathcal{P})$ if $\mathcal{P}$ is a finite collection of finite groups (more generally, of groups $P$ with $\operatorname{Out}(P)$ finite).

If $\mathcal{H}=\left\{H_{1}, \ldots, H_{q}\right\}$ is another family of subgroups, we define

$$
\operatorname{Out}\left(G ; \mathcal{P}, \mathcal{H}^{(\mathrm{t})}\right)=\operatorname{Out}(G ; \mathcal{P}) \cap \operatorname{Out}\left(G ; \mathcal{H}^{(\mathrm{t})}\right) .
$$

We allow $\mathcal{P}$ or $\mathcal{H}$ to be empty, in which case we do not write it.

The groups defined above do not change if we replace each $P_{i}$ or $H_{j}$ by a conjugate, or if we add conjugates of the $P_{i}$ 's to $\mathcal{P}$ or conjugates of the $H_{j}$ 's to $\mathcal{H}$.

2.2. Splittings. A splitting of a group $G$ is an isomorphism between $G$ and the fundamental group of a graph of groups $\Gamma$. Equivalently, using Bass-Serre theory, we view a splitting of $G$ as an action of $G$ on a simplicial tree $T$, with $T / G=\Gamma$. This tree is well-defined up to equivariant isomorphism, and two splittings are considered equal if there is an equivariant isomorphism between the corresponding Bass-Serre trees.

The group $\operatorname{Out}(G)$ acts on the set of splittings of $G$ (by changing the isomorphism between $G$ and $\pi_{1}(\Gamma)$, or precomposing the action on $T$ ).

Trees will always be simplicial trees with an action of $G$ without inversion. We usually assume that the splitting is minimal (there is no proper $G$-invariant subtree). Since $G$ is assumed to be finitely generated, this implies that $\Gamma$ is a finite graph.

A splitting is trivial if $G$ fixes a point in $T$ (minimality then implies that $T$ is a point).

A splitting is relative to $\mathcal{P}$ if every $P_{i}$ is conjugate to a subgroup of a vertex group, or equivalently if $P_{i}$ is elliptic (i.e. fixes a point) in $T$. If $\mathcal{P}=\left\{P_{1}, \ldots, P_{n}\right\}$, we also say that the splitting is relative to $P_{1}, \ldots, P_{n}$. 
The group $G$ splits over a subgroup $A$ (relative to $\mathcal{P}$ ) if there is a non-trivial minimal splitting (relative to $\mathcal{P}$ ) such that $A$ is an edge group. The group is oneended relative to $\mathcal{P}$ if it does not split over a finite group relative to $\mathcal{P}$.

If $\Gamma$ is a graph of groups, we denote by $V$ its set of vertices, and by $E$ its set of oriented edges. The origin of an edge $e \in E$ is denoted by $o(e)$. A vertex $v$ or an edge $e$ carries a group $G_{v}$ or $G_{e}$, and there is an inclusion $i_{e}: G_{e} \rightarrow G_{o(e)}$

2.3. Trees and deformation spaces $[24,32]$. In this subsection we consider trees rather than graphs of groups. We denote by $G_{v}$ or $G_{e}$ the stabilizer of a vertex or an edge.

We often restrict edge stabilizers of $T$ by requiring that they belong to a family $\mathcal{A}$ of subgroups of $G$ which is stable under taking subgroups and under conjugation. We then say that $T$ is an $\mathcal{A}$-tree. For instance, $\mathcal{A}$ may be the set of finite subgroups, of cyclic subgroups, of abelian subgroups, of elementary subgroups of a relatively hyperbolic group (see Section 3). We then speak of cyclic, abelian, elementary trees (or splittings).

Besides restricting edge stabilizers, we also often restrict to trees $T$ relative to $\mathcal{P}$ : every $P_{i}$ is elliptic in $T$. We then say that $T$ is an $(\mathcal{A}, \mathcal{P})$-tree.

A tree $T^{\prime}$ is a collapse of $T$ if it is obtained from $T$ by collapsing each edge in a certain $G$-invariant collection to a point; conversely, we say that $T$ refines $T^{\prime}$. In terms of graphs of groups, one passes from $\Gamma=T / G$ to $\Gamma^{\prime}=T^{\prime} / G$ by collapsing edges; for each vertex $v^{\prime} \in \Gamma^{\prime}$, the vertex group $G_{v^{\prime}}$ is the fundamental group of the graph of groups occuring as the preimage of $v^{\prime}$ in $\Gamma$.

Given two trees $T$ and $T^{\prime}$, we say that $T$ dominates $T^{\prime}$ if there is a $G$-equivariant map $f: T \rightarrow T^{\prime}$, or equivalently if every subgroup which is elliptic in $T$ is also elliptic in $T^{\prime}$. In particular, $T$ dominates any collapse $T^{\prime}$.

Two trees belong to the same deformation space if they dominate each other. In other words, a deformation space $\mathcal{D}$ is the set of all trees having a given family of subgroups as their elliptic subgroups. We denote by $\mathcal{D}(T)$ the deformation space containing a tree $T$, and by $\operatorname{Out}(\mathcal{D}) \subset \operatorname{Out}(G)$ the group of automorphisms leaving $\mathcal{D}$ invariant. The set of $\mathcal{A}$-trees contained in a deformation space is called a deformation space over $\mathcal{A}$ (and usually denoted by $\mathcal{D}$ also).

A tree is reduced if $G_{e} \neq G_{v}, G_{w}$ whenever an edge $e$ has its endpoints $v, w$ in different $G$-orbits. Equivalently, no tree obtained from $T$ by collapsing the orbit of an edge belongs to the same deformation space as $T$. If $T$ is not reduced, one may collapse edges so as to obtain a reduced tree in the same deformation space.

Any two reduced trees in a deformation space over finite groups may be joined by slide moves (see $[24,32]$ for definitions). In particular, they have the same set of edge and vertex stabilizers. 


\subsection{Induced structures}

Definition 2.1 (Incident edge groups $\operatorname{Inc}_{v}$ ). Given a vertex $v$ of a graph of groups $\Gamma$, we denote by $\operatorname{Inc}_{v}$ the collection of all subgroups $i_{e}\left(G_{e}\right)$ of $G_{v}$, for $e$ an edge with origin $v$. We call $\operatorname{Inc}_{v}$ the set of incident edge groups. We also use the notation $\operatorname{Inc}_{G_{v}}$.

Similarly, if $v$ is a vertex of a (minimal) tree, there are finitely many $G_{v}$-orbits of incident edges, and $\mathrm{Inc}_{v}$ is the family of stabilizers of some representatives of these orbits. This is a finite collection of subgroups of $G_{v}$, each well-defined up to conjugacy.

Any splitting $\Gamma_{v}$ of $G_{v}$ relative to Inc ${ }_{v}$ extends (non-uniquely) to a splitting $\Lambda$ of $G$, whose edges are those of $\Gamma_{v}$ together with those of $\Gamma$; an edge $e$ of $\Gamma$ incident to $v$ is attached to a vertex of $\Gamma_{v}$ whose group contains $G_{e}$ (up to conjugacy). We call this refining $\Gamma$ at $v$ using $\Gamma_{v}$. One recovers $\Gamma$ from $\Lambda$ by collapsing edges of $\Gamma_{v}$.

Lemma 2.2. Consider subgroups $H \subset K \subset G$ such that, if $g \in G$ and $H^{g} \subset K$, then $g \in K$ (this holds in particular when $K$ is a vertex stabilizer of a tree $T$, and $H$ is a subgroup which fixes no edge of $T$ ).

- If $H^{\prime} \subset K$ is conjugate to $H$ in $G$, it is conjugate to $H$ in $K$.

- If $\alpha \in \operatorname{Aut}(G)$ leaves $K$ invariant and maps $H$ to $g \mathrm{Hg}^{-1}$, then $g \in K$.

This lemma is trivial, but very useful. Given a vertex stabilizer $G_{v}$ of a tree $T$, it allows us to define a family $\mathcal{P}_{\mid G_{v}}$ as follows (like $\operatorname{Inc}_{v}$, it is a finite set of subgroups of $G_{v}$, each well-defined up to conjugacy).

Definition 2.3 (Induced structure $\mathcal{P}_{\mid G_{v}}$ ). Let $\mathcal{P}=\left\{P_{i}\right\}$ be a collection of subgroups of $G$, and let $G_{v}$ be a vertex stabilizer in a tree $T$ relative to $\mathcal{P}$. For each $i$ such that $P_{i}$ fixes a point in the orbit of $v$, but fixes no edge of $T$, let $\widetilde{P}_{i} \subset G_{v}$ be a conjugate of $P_{i}$. When defined, $\widetilde{P}_{i}$ is unique up to conjugacy in $G_{v}$ by Lemma 2.2. We define $\mathcal{P}_{\mid G_{v}}$ as this collection of subgroups $\widetilde{P}_{i} \subset G_{v}$; we define $\mathcal{P}_{\mid G_{v}}$ similarly if $G_{v}$ is a vertex group of $\Gamma=T / G$.

Remark 2.4. Given $v$ and $i$, one of the following always holds: $P_{i}$ fixes a vertex of $T$ not in the orbit of $v$, or some conjugate of $P_{i}$ fixes $v$ and an edge incident to $v$, or $P_{i}$ is conjugate to a group in $\mathcal{P}_{\mid G_{v}}$.

Remark 2.5. In this definition, $\mathcal{P}_{\mid G_{v}}$ depends not only on $\mathcal{P}$ and $G_{v}$, but also on the incident edge groups of $G_{v}$. In practice, we will not work with $\mathcal{P}_{\mid G_{v}}$ alone, but with $Q_{v}=\mathcal{P}_{\mid G_{v}} \cup$ Inc $_{v}$. This is the case for instance in the following lemma. 
Lemma 2.6. If $\Gamma_{v}$ is a splitting of $G_{v}$ relative to $Q_{v}=\operatorname{Inc}_{v} \cup \mathcal{P}_{\mid G_{v}}$, refining $\Gamma$ at $v$ using $\Gamma_{v}$ yields a splitting $\Lambda$ of $G$ which is relative to $\mathcal{P}$.

Proof. The refinement is possible because $\Gamma_{v}$ is relative to $\operatorname{Inc}_{v}$. Each $P_{i}$ is elliptic in $\Lambda$ by Remark 2.4 .

2.5. JSJ decompositions [35]. Fix $\mathcal{A}$ as in Subsection 2.3, and a (possibly empty) family $\mathcal{P}$ of subgroups. All trees considered here are $(\mathcal{A}, \mathcal{P})$-trees.

A subgroup $H$ is universally elliptic if it is elliptic (fixes a point) in every tree. A tree is universally elliptic if its edge stabilizers are. A tree $T$ is a $J S J$ tree over $\mathcal{A}$ relative to $\mathcal{P}$ if it is universally elliptic and dominates every universally elliptic tree (see Section 4 of [35]). JSJ trees exist if $G$ is finitely presented relative to $\mathcal{P}$. The set of JSJ trees, if non-empty, is a deformation space called the JSJ deformation space over $\mathcal{A}$ relative to $\mathcal{P}$. When $\mathcal{A}$ is the set of cyclic (abelian, elementary...) subgroups, we refer to the cyclic (abelian, elementary...) JSJ.

When $\mathcal{A}$ is the set of finite subgroups, and $\mathcal{P}=\emptyset$, the JSJ deformation space is the Stallings-Dunwoody deformation space, characterized by the property that its trees have vertex stabilizers with at most one end (see Section 6 of [35]). Because it is a deformation space over finite groups, all its reduced trees have the same edge and vertex stabilizers (see Subsection 2.3). This space exists if and only if $G$ is accessible, in particular when $G$ is finitely presented [22]. If $G$ is torsion-free, the Stallings-Dunwoody deformation space is the Grushko deformation space; edge stabilizers are trivial, vertex stabilizers are freely indecomposable and non-cyclic.

More generally, the JSJ deformation space over finite subgroups relative to $\mathcal{P}$ will be called the Stallings-Dunwoody deformation space relative to $\mathcal{P}$. We will also consider JSJ spaces over finite subgroups of cardinality bounded by some $k$; these exist whenever $G$ is finitely generated by Linnell's accessibility [49].

If $T$ is a tree (in particular, if it is a JSJ tree), a vertex stabilizer $G_{v}$ of $T$ not belonging to $\mathcal{A}$ (or $v$ itself) is rigid if it is universally elliptic. Otherwise, $G_{v}$ (or $v$ ) is flexible. In many situations, flexible vertex stabilizers $G_{v}$ of JSJ trees are quadratically hanging subgroups (see Section 7 of [35]).

Definition 2.7 (QH vertex). A vertex stabilizer $G_{v}$ (or $\left.v\right)$ is quadratically hanging, or $Q H$, (relative to $\mathcal{P}$ ) if there is a normal subgroup $F \triangleleft G_{v}$ (called the fiber of $G_{v}$ ) such that $G_{v} / F$ is isomorphic to the fundamental group $\pi_{1}(\Sigma)$ of a compact hyperbolic 2-orbifold $\Sigma$ (usually with boundary); moreover, if $H \subset G_{v}$ is an incident edge stabilizer, or is the intersection of $G_{v}$ with a conjugate of a group in $\mathcal{P}$, then the image of $H$ in $\pi_{1}(\Sigma)$ is finite or contained in a boundary subgroup (a subgroup conjugate to the fundamental group of a boundary component). 
Definition 2.8 (Full boundary subgroups). Let $G_{v}$ be a QH vertex stabilizer. For each boundary component of $\Sigma$, we select a representative for the conjugacy class of its fundamental group in $\pi_{1}(\Sigma)$, and we consider its full preimage in $G_{v}$. This defines a finite family $\mathcal{B}_{v}$ of subgroups of $G_{v}$.

If $G_{v}$ is QH with finite fiber, every infinite incident edge stabilizer is virtually cyclic and (up to conjugacy in $G_{v}$ ) contained with finite index in a group of $\mathcal{B}_{v}$. If $G$ is one-ended relative to $\mathcal{P}$, then every incident edge stabilizer is infinite, so is contained in a group of $\mathcal{B}_{v}$.

Remark 2.9. If $G_{v}$ is a flexible $\mathrm{QH}$ vertex stabilizer with finite fiber, and $H \subset G_{v}$ is universally elliptic, then the image of $H$ in $\pi_{1}(\Sigma)$ is finite or contained in a boundary subgroup (see Proposition 7.6 of [35]; this requires a technical assumption on $\mathcal{A}$, which holds in all cases considered in the present paper). In particular, $H$ is virtually cyclic.

2.6. The automorphism group of a tree [45]. Let $T$ be a tree with a minimal action of $G$. We assume that $T$ is not a line with $G$ acting by translations.

We denote by $\operatorname{Aut}(T) \subset \operatorname{Aut}(G)$ the group of automorphisms $\alpha$ leaving $T$ invariant: there exists an isomorphism $f_{\alpha}: T \rightarrow T$ which is $\alpha$-equivariant in the sense that $f_{\alpha}(g x)=\alpha(g) f_{\alpha}(x)$ for $g \in G$ and $x \in T$.

Following [45], we describe the image $\operatorname{Out}(T)$ of $\operatorname{Aut}(T)$ in $\operatorname{Out}(G)$ in terms of the graph of groups $\Gamma=T / G$. Our assumptions on $T$ imply that $\Gamma$ is minimal, and is not a mapping torus (as defined in [45]).

The group $\operatorname{Out}(T)$ acts on the finite graph $\Gamma=T / G$, and we define $\operatorname{Out}^{0}(T)$ as the finite index subgroup acting trivially. We use the notations

$$
\operatorname{Out}\left(T ; \mathcal{P}, \mathcal{H}^{(\mathrm{t})}\right)=\operatorname{Out}(T) \cap \operatorname{Out}\left(G ; \mathcal{P}, \mathcal{H}^{(\mathrm{t})}\right),
$$

and

$$
\operatorname{Out}^{0}\left(T ; \mathcal{P}, \mathcal{H}^{(\mathrm{t})}\right)=\operatorname{Out}^{0}(T) \cap \operatorname{Out}\left(G ; \mathcal{P}, \mathcal{H}^{(\mathrm{t})}\right)
$$

(see Section 2.1).

Action on vertex groups. If $v \in V$ is a vertex of $\Gamma$, there is a natural map

$$
\rho_{v}: \operatorname{Out}^{0}(T) \longrightarrow \operatorname{Out}\left(G_{v}\right)
$$

defined as follows. Let $\Phi \in \operatorname{Out}^{0}(T)$. When $N_{G}\left(G_{v}\right)$ acts on $G_{v}$ by inner automorphisms, in particular when $G_{v}$ fixes a unique point in $T$ (in this case $N_{G}\left(G_{v}\right)=$ $G_{v}$ ), one defines $\rho_{v}(\Phi)$ simply by choosing any representative $\alpha \in \operatorname{Aut}(T)$ of $\Phi$ 
leaving $G_{v}$ invariant and considering its restriction to $G_{v}$. In general, one has to choose $\alpha$ more carefully: one fixes a vertex $\tilde{v}$ of $T$ mapping to $v$ such that the stabilizer of $\tilde{v}$ is $G_{v}$, one chooses $\alpha$ so that $f_{\alpha}$ fixes $\tilde{v}$, and $\rho_{v}(\Phi)$ is represented by the restriction $\alpha_{\mid G_{v}}$.

If $e$ is an edge of $\Gamma$, one may define

$$
\rho_{e}: \operatorname{Out}^{0}(T) \longrightarrow \operatorname{Out}\left(G_{e}\right)
$$

similarly.

Let

$$
\rho: \operatorname{Out}^{0}(T) \longrightarrow \prod_{v \in V} \operatorname{Out}\left(G_{v}\right)
$$

be the product map. As observed in Subsection 2.3 of [45], the image $\rho\left(\operatorname{Out}^{0}(T)\right)$ contains $\prod_{v \in V} \operatorname{Out}\left(G_{v} ; \operatorname{Inc}_{v}^{(\mathrm{t})}\right)$ and is contained in $\prod_{v \in V} \operatorname{Out}\left(G_{v} ; \operatorname{Inc}_{v}\right)$. More precisely:

Lemma 2.10. Let $\mathcal{P}, \mathcal{H}$ be two families of subgroups of $G$. Let $T$ be a tree relative to $\mathcal{P} \cup \mathcal{H}$. Then

$$
\begin{aligned}
\operatorname{Out}\left(G_{v} ; \operatorname{Inc}_{v}^{(\mathrm{t})}, \mathcal{P}_{\mid G_{v}}, \mathcal{H}_{\mid G_{v}}^{(\mathrm{t})}\right) & \subset \rho_{v}\left(\operatorname{Out}^{0}\left(T ; \mathcal{P}, \mathcal{H}^{(\mathrm{t})}\right)\right) \\
& \subset \operatorname{Out}\left(G_{v} ; \operatorname{Inc}_{v}, \mathcal{P}_{\mid G_{v}}, \mathcal{H}_{\mid G_{v}}^{(\mathrm{t})}\right)
\end{aligned}
$$

for every $v \in V$, and

$$
\begin{aligned}
\prod_{v \in V} \operatorname{Out}\left(G_{v} ; \operatorname{Inc}_{v}^{(\mathrm{t})}, \mathcal{P}_{\mid G_{v}}, \mathcal{H}_{\mid G_{v}}^{(\mathrm{t})}\right) & \subset \rho\left(\operatorname{Out}^{0}\left(T ; \mathcal{P}, \mathcal{H}^{(\mathrm{t})}\right)\right) \\
& \subset \prod_{v \in V} \operatorname{Out}\left(G_{v} ; \operatorname{Inc}_{v}, \mathcal{P}_{\mid G_{v}}, \mathcal{H}_{\mid G_{v}}^{(\mathrm{t})}\right) .
\end{aligned}
$$

If $\operatorname{Out}\left(G_{e}\right)$ is finite for all edges (resp. for all edges e incident to $v$ ), all inclusions (resp. all inclusions in (1)) have images of finite index.

Recall that $\mathcal{P}_{\mid G_{v}}$ was defined in Definition 2.3.

Proof. The inclusion $\prod_{v \in V} \operatorname{Out}\left(G_{v} ; \operatorname{Inc}_{v}^{(\mathrm{t})}\right) \subset \rho\left(\operatorname{Out}^{0}(T)\right)$ is proved in [45] by extending any $\Phi_{v} \in \operatorname{Out}\left(G_{v} ; \operatorname{Inc}_{v}^{(\mathrm{t})}\right)$ "by the identity" to get $\Phi \in \operatorname{Out}^{0}(T)$, with $\Phi_{v}=\rho_{v}(\Phi)$, acting as a conjugation on each edge group and on each $G_{w}$ for $w \neq v$. The left hand side inclusions in the lemma follow from Remark 2.4.

The inclusion $\rho_{v}\left(\operatorname{Out}^{0}(T)\right) \subset \operatorname{Out}\left(G_{v} ; \operatorname{Inc}_{v}\right)$ follows from the fact that, given an edge $e$ of $T$ containing the lift $\tilde{v}$ of $v$ used to define $\rho_{v}$, any $\Phi \in \operatorname{Out}^{0}(T)$ has 
a representative $\alpha$ such that $f_{\alpha}$ fixes $e$; this representative induces an automorphism of $G_{\tilde{v}}$ leaving $G_{e}$ invariant. To prove the right hand side inclusions, apply Lemma 2.2 with $K=G_{\tilde{v}}$, recalling that groups in $\mathcal{H}_{\mid G_{v}}$ or $\mathcal{P}_{\mid G_{v}}$ fix a unique point in $T$.

If $\operatorname{Out}\left(G_{e}\right)$ is finite for all incident edge $\operatorname{groups}, \operatorname{Out}\left(G_{v} ; \operatorname{Inc}_{v}^{(\mathrm{t})}\right)$ has finite index in $\operatorname{Out}\left(G_{v} ; \operatorname{Inc}_{v}\right)$ (see Proposition 2.3 in [45]). Considering the intersection with $\operatorname{Out}\left(G_{v} ; \mathcal{P}_{\mid G_{v}}, \mathcal{H}_{\mid G_{v}}^{(\mathrm{t})}\right)$, we get that $\operatorname{Out}\left(G_{v} ; \operatorname{Inc}_{v}^{(\mathrm{t})}, \mathcal{P}_{\mid G_{v}}, \mathcal{H}_{\mid G_{v}}^{(\mathrm{t})}\right)$ has finite index in $\operatorname{Out}\left(G_{v} ; \operatorname{Inc}_{v}, \mathcal{P}_{\mid G_{v}}, \mathcal{H}_{\mid G_{v}}^{(\mathrm{t})}\right)$. This concludes the proof.

Remark 2.11. We can also consider automorphisms which do not leave $T$ invariant, but only leave some vertex stabilizer $G_{v}$ invariant. Assuming that $G_{v}$ equals its normalizer, there is a natural map

$$
\rho_{v}: \operatorname{Out}\left(G ; G_{v}\right) \longrightarrow \operatorname{Out}\left(G_{v}\right)
$$

and

$$
\operatorname{Out}\left(G_{v} ; \operatorname{Inc}_{v}^{(\mathrm{t})}, \mathcal{P}_{\mid G_{v}}, \mathcal{H}_{\mid G_{v}}^{(\mathrm{t})}\right) \subset \rho_{v}\left(\operatorname{Out}\left(G ; G_{v}, \mathcal{P}, \mathcal{H}^{(\mathrm{t})}\right)\right) \subset \operatorname{Out}\left(G_{v} ; \mathcal{P}_{\mid G_{v}}, \mathcal{H}_{\mid G_{v}}^{(\mathrm{t})}\right)
$$

Twists. As in Subsection 2.5 of [45], we now consider the kernel of the product map $\rho: \operatorname{Out}^{0}(T) \rightarrow \prod_{v \in V} \operatorname{Out}\left(G_{v}\right)$. It consists of automorphisms in $\operatorname{Out}^{0}(T)$ having, for each $v$, a representative in $\operatorname{Aut}(T)$ whose restriction to $G_{v}$ is the identity. If $T$ is relative to $\mathcal{P}$, the group $\operatorname{ker} \rho$ is contained in $\operatorname{Out}\left(T ; \mathcal{P}^{(\mathrm{t})}\right)$.

To study $\operatorname{ker} \rho$, we need to introduce the group of twists $\mathcal{T}$ associated to $T$ or equivalently to $\Gamma$ (we write $\mathcal{T}(T)$ or $\mathcal{T}(\Gamma)$ if there is a risk of confusion).

Let $e$ be a separating edge of $\Gamma$ with origin $v$ and endpoint $w$. Then $G=$ $A *_{G_{e}} B$ with $G_{v} \subset A$ and $G_{w} \subset B$. Given $g \in Z_{G_{v}}\left(G_{e}\right)$, one defines the twist by $g$ around e near $v$ as the (image in $\operatorname{Out}(G)$ of the) automorphism of $G$ equal to the identity on $B$ and to conjugation by $g$ on $A$. There is a similar definition in the case of an HNN extension $G=A *_{C}=\left\langle A, t \mid t c t^{-1}=\varphi(c), c \in C\right\rangle$ : given $g \in Z_{A}(C)$, the twist by $g$ is the identity on $A$ and sends $t$ to $t g$.

The group $\mathcal{T}$ is the subgroup of $\operatorname{Out}(G)$ generated by all twists. It is a quotient of $\prod_{e \in E} Z_{G_{o(e)}}\left(G_{e}\right)$ and is contained in $\operatorname{ker} \rho$. The following facts follow directly from Section 2 of [45].

Lemma 2.12. (1) If every $\operatorname{Out}\left(G_{e}\right)$ is finite, then $\mathcal{T}$ has finite index in $\operatorname{ker} \rho$.

(2) Assume that every non-oriented edge e of $\Gamma$ has an endpoint $v$ such that $N_{G_{v}}\left(G_{e}\right)$ acts on $G_{e}$ by inner automorphisms (this holds in particular if $G_{v}$ is abelian, or if $G_{e}$ is malnormal in $G_{v}$, or if $G_{e}$ is infinite and almost malnormal in $\left.G_{v}\right)$. Then $\mathcal{T}=\operatorname{ker} \rho$. 
The kernel of the epimorphism from $\prod_{e \in E} Z_{G_{o(e)}}\left(G_{e}\right)$ to $\mathcal{T}$ is the image of a natural map

$$
j: \prod_{v \in V} Z\left(G_{v}\right) \times \prod_{e \in \mathcal{E}} Z\left(G_{e}\right) \longrightarrow \prod_{e \in E} Z_{G_{o(e)}}\left(G_{e}\right)
$$

where $\mathcal{E}$ is the set of non-oriented edges of $\Gamma$ (see Proposition 3.1 of [45]). The image of an element of $Z\left(G_{v}\right)$ is called a vertex relation at $v$, the image of an element of $Z\left(G_{e}\right)$ is an edge relation.

For instance, if $\Gamma$ is a non-trivial amalgam $G=A *_{C} B$, then $\mathcal{T}$ is the image of the map $p: Z_{A}(C) \times Z_{B}(C) \rightarrow \operatorname{Out}(G)$ sending $(a, b)$ to the class of the automorphism acting on $A$ as conjugation by $a$ and on $B$ as conjugation by $b$. The kernel of $p$ is generated by the elements $(a, 1)$ with $a \in Z(A)$ and $(1, b)$ with $b \in Z(B)$ (vertex relations), together with the elements $(c, c)$ with $c \in Z(C)$ (edge relations).

Lemma 2.13. Lete be an edge of $\Gamma$ with origin v. If $Z\left(G_{e}\right)$ and $Z\left(G_{v}\right)$ are finite, but $Z_{G_{v}}\left(G_{e}\right)$ is infinite, then the image of $Z_{G_{v}}\left(G_{e}\right)$ in $\mathcal{T}$ is infinite. In particular, $\mathcal{T}$ is infinite.

Note that $Z_{G_{v}}\left(G_{e}\right)$ is infinite if $N_{G_{v}}\left(G_{e}\right)$ is infinite and $G_{e}$ is finite.

Proof. It is pointed out in [47, Lemma 3.2] that the image of $Z_{G_{v}}\left(G_{e}\right)$ in $\mathcal{T}$ maps onto the quotient $Z_{G_{v}}\left(G_{e}\right) /\left\langle Z\left(G_{v}\right), Z\left(G_{e}\right)\right\rangle$. Since $Z\left(G_{v}\right)$ and $Z\left(G_{e}\right)$ are commuting finite subgroups, this quotient is infinite.

Let $\Gamma$ be a graph of groups with fundamental group $G$, and $\Gamma_{0} \subset \Gamma$ a connected subgraph. We view $\Gamma_{0}$ as a graph of groups, with fundamental group $G_{0} \subset G$ and associated group of twists $\mathcal{T}\left(\Gamma_{0}\right) \subset \operatorname{Out}\left(G_{0}\right)$.

Lemma 2.14. If $\mathcal{T}\left(\Gamma_{0}\right)$ is infinite, then so is $\mathcal{T}(\Gamma)$.

Proof. Let $E_{0}$ be the set of oriented edges of $\Gamma_{0}$. Consider the projection from $\prod_{e \in E} Z_{G_{o(e)}}\left(G_{e}\right)$ to $\prod_{e \in E_{0}} Z_{G_{o(e)}}\left(G_{e}\right)$ obtained by keeping only the factors with $e \subset \Gamma_{0}$. It is compatible with the vertex and edge relations, so induces an epimorphism from $\mathcal{T}(\Gamma)$ to $\mathcal{T}\left(\Gamma_{0}\right)$.

Lemma 2.15. If $\Gamma$ is a graph of groups with $\mathcal{T}(\Gamma)$ infinite, there is an edge e such that the graph of groups $\Gamma_{e}$ obtained by collapsing every edge except e has $\mathcal{T}\left(\Gamma_{e}\right)$ infinite.

Proof. There is an edge $e$ such that $Z_{G_{o(e)}}\left(G_{e}\right)$ has infinite image in $\operatorname{Out}(G)$. Twists of $\Gamma$ around $e$ are also twists of $\Gamma_{e}$. 
2.7. Trees of cylinders [34]. We recall some basic properties of the tree of cylinders (see Section 4 of [34] for details). Besides $\mathcal{A}$ (and possibly $\mathcal{P}$ ), we have to fix a conjugacy-invariant subfamily $\mathcal{E} \subset \mathcal{A}$ and an admissible equivalence relation $\sim$ on $\mathcal{E}$. Rather than giving a general definition, we describe the examples that will be used in this paper (with $\mathcal{A}$ consisting of all finite, elementary, or abelian subgroups respectively):

(1) $\mathcal{E}$ consists of all finite subgroups of a fixed order $k$, and $\sim$ is equality;

(2) $G$ is relatively hyperbolic (see Section 3 ), $\mathcal{E}$ consists of all infinite elementary subgroups (parabolic or loxodromic), and $\sim$ is co-elementarity: $A \sim B$ if and only if $\langle A, B\rangle$ is elementary;

(3) $G$ is a torsion-free CSA group, $\mathcal{E}$ consists of all infinite abelian subgroups, and $\sim$ is commutation: $A \sim B$ if and only if $\langle A, B\rangle$ is abelian (recall that $G$ is CSA if centralizers of non-trivial elements are abelian and malnormal).

Let $T$ be a tree with edge stabilizers in $\mathcal{E}$. We declare two (non-oriented) edges $e$ and $f$ to be equivalent if $G_{e} \sim G_{f}$. The union of all edges in an equivalence class is a subtree $Y$, called a cylinder of $T$. Two distinct cylinders meet in at most one point. The tree of cylinders $T_{c}$ of $T$ is the bipartite tree such that $V_{0}\left(T_{c}\right)$ is the set of vertices $x$ of $T$ which belong to at least two cylinders, $V_{1}\left(T_{c}\right)$ is the set of cylinders $Y$ of $T$, and there is an edge $\varepsilon=(x, Y)$ between $x$ and $Y$ in $T_{c}$ if and only if $x \in Y$. In other words, one obtains $T_{c}$ from $T$ by replacing each cylinder $Y$ by the cone on its boundary (defined as the set of vertices of $Y$ belonging to some other cylinder). Note that $T_{c}$ may be trivial even if $T$ is not.

The tree $T_{c}$ is dominated by $T$ (in particular, it is relative to $\mathcal{P}$ if $T$ is). It only depends on the deformation space $\mathcal{D}$ containing $T$ (we sometimes say that it is the tree of cylinders of $\mathcal{D}$ ). In particular, $T_{c}$ is invariant under any automorphism of $G$ leaving $\mathcal{D}$ invariant.

The stabilizer of a vertex $x \in V_{0}\left(T_{c}\right)$ is the stabilizer of $x$, viewed as a vertex of $T$. The stabilizer $G_{Y}$ of a vertex $Y \in V_{1}\left(T_{c}\right)$ is the stabilizer $G_{\mathcal{C}}$ of the equivalence class $\mathcal{C} \in \mathcal{E} / \sim$ containing stabilizers of edges in $Y$, for the action of $G$ on $\mathcal{E}$ by conjugation (see Subsection 5.1 of [34]). It is the normalizer of a finite subgroup in case 1, a maximal elementary (resp. abelian) subgroup in case 2 (resp. 3). Note that $A \subset G_{\mathcal{C}}$ if $A \in \mathcal{E}$ and $\mathcal{C}$ is its equivalence class.

The stabilizer of an edge $\varepsilon=(x, Y)$ of $T_{c}$ is $G_{\varepsilon}=G_{x} \cap G_{Y}$; it is elliptic in $T$. In cases 2 and $3, G_{\varepsilon}$ belongs to $\mathcal{A}$. But, in case 1, it may happen that edge stabilizers of $T_{c}$ are not in $\mathcal{A}$, so we also consider the collapsed tree of cylinders $T_{c}^{*}$ obtained from $T_{c}$ by collapsing each edge whose stabilizer does not belong to $\mathcal{A}$ (see Subsection 5.2 of [34]). It is an $(\mathcal{A}, \mathcal{P})$-tree if $T$ is, and $\left(T_{c}^{*}\right)_{c}^{*}=T_{c}^{*}$. 


\section{Relatively hyperbolic groups}

In this section we assume that $G$ is hyperbolic relative to a finite family $\mathcal{P}=$ $\left\{P_{1}, \ldots, P_{n}\right\}$ of finitely generated subgroups; we say that $\mathcal{P}$ is the parabolic structure.

The group $G$ is finitely generated. It is not necessarily finitely presented, but it is finitely presented relative to $\mathcal{P}$ [53], so JSJ decompositions relative to $\mathcal{P}$ exist. In particular, there is a deformation space of relative Stallings-Dunwoody decompositions (see Subsection 2.5).

3.1. Generalities. We refer to [39] for equivalent definitions of relative hyperbolicity. In particular, $G$ acts properly discontinuously on a proper geodesic $\delta$-hyperbolic space $X$ (which may be taken to be a graph [28]), the action is cocompact in the complement of a $G$-invariant union $\mathcal{B}$ of disjoint horoballs, and the $P_{i}$ 's are representatives of conjugacy classes of stabilizers of horoballs. Any horoball $B \in \mathcal{B}$ has a unique point at infinity $\xi$, and the stabilizer of $\xi$ (for the action of $G$ on $\partial X$ ) coincides with the stabilizer of $B$.

For each constant $M>0$, one can change the system of horoballs so that any two distinct horoballs are at distance at least $M$. Indeed, for each horoball $B$ with stabilizer $P$ defined by a horofunction $h$, the function $h^{\prime}(x)=\sup _{g \in P} h(g x)$ is another (well-defined) horofunction which is $P$-equivariant; then $B^{\prime}=h^{\prime-1}([R, \infty))$ is a new $P$-invariant horoball such that $d\left(B^{\prime}, X \backslash B\right) \geq M$ for $R$ large enough. Doing this for a chosen horoball in each orbit, and extending by equivariance, one gets a system of horoballs at distance at least $M$ from each other.

A subgroup of $G$ is parabolic if it is contained in a conjugate of some $P_{i}$, loxodromic if it is infinite, virtually cyclic, and not parabolic, elementary if it is parabolic or virtually cyclic (finite or loxodromic). Any small subgroup is elementary. The group $G$ itself is elementary if it is virtually cyclic or equal to a $P_{i}$. We say that $A$ is an elementary subgroup of $B$ if it is elementary and contained in $B$.

One may remove any virtually cyclic subgroup from $\mathcal{P}$, without destroying relative hyperbolicity (see e.g. [20, Cor 1.14]). Conversely, one may add to $\mathcal{P}$ a finite subgroup or a maximal loxodromic subgroup (see e.g. [52]). These operations do not change the set of elementary (or relatively quasiconvex, as defined below) subgroups, and it is sometimes convenient (as in [39]) to assume that every $P_{i}$ is infinite. Any infinite $P_{i}$ is a maximal elementary subgroup.

The following lemma is folklore, but we have not found it in the literature. 
Lemma 3.1. Given a relatively hyperbolic group $G$, there exists a number $M$ such that any elementary subgroup $H \subset G$ of cardinality $>M$ is contained in a unique maximal elementary subgroup $E(H)$. There are finitely many conjugacy classes of non-parabolic finite subgroups.

Proof. We may assume that every $P_{i}$ is infinite. Let $H$ be elementary. The existence of $E(H)$ is well-known if $H$ is infinite (see for instance [53]), so assume $H$ is finite. We also assume that the distance between any two distinct horoballs in $\mathcal{B}$ is bigger than $6 \delta$. Given $r>0$, let $X_{r}$ be the set of points of $X$ which are moved less than $r$ by $H$. It follows from Lemma 3.3 p. 460 of [7] (existence of quasi-centres) that $X_{5 \delta}$ is nonempty.

Arguing as in the proof of Lemma 1.15 page 407 and Lemma 3.3 page 428 in [7], one sees that any geodesic joining two points of $X_{58}$, or a point of $X_{58}$ to a fixed point of $H$ in $\partial X$, is contained in $X_{98}$.

If $X_{98}$ meets $X \backslash \mathcal{B}$, properness of the action of $G$ on $X \backslash \mathcal{B}$ implies that there are only finitely many possibilities for $H$ up to conjugacy, so we can choose $M$ to ensure $X_{9 \delta} \subset \mathcal{B}$. This implies that $X_{5 \delta}$ is contained in a unique horoball $B_{0}$ of $\mathcal{B}$. This horoball is $H$-invariant since horoballs are $6 \delta$-apart, so $H$ fixes the point at infinity $\xi_{0}$ of $B_{0}$ and is contained in the maximal parabolic subgroup $E(H)=\operatorname{Stab}\left(B_{0}\right)$. In particular, $H$ is parabolic.

There remains to prove uniqueness of $E(H)$. It suffices to check that $H$ cannot fix any point $\xi \neq \xi_{0}$ in $\partial X$. If it did, a geodesic joining $\xi$ to a point of $X_{58}$ would be contained in $X_{98}$ and meet $X \backslash \mathcal{B}$. This contradicts our choice of $M$.

Since maximal elementary subgroups are equal to their normalizer, we get:

Corollary 3.2 ([21, Lemma 4.20]). Maximal elementary subgroups $E$ are uniformly almost malnormal: if $E \cap g E g^{-1}$ has cardinality $>M$, then $g \in E$.

\subsection{Quasiconvexity}

Definition 3.3 (Hruska [39]). Let $X$ and $\mathcal{B}$ be as above, and $C>0$. A subspace $Y \subset X$ is relatively $C$-quasiconvex if, given $y, y^{\prime} \in Y$, any geodesic $\left[y, y^{\prime}\right] \subset X$ has the property that $\left[y, y^{\prime}\right] \backslash \mathcal{B}$ lies in the $C$-neighbourhood of $Y$. The space $Y$ is relatively quasiconvex if it is relatively $C$-quasiconvex for some $C$. A subgroup $H<G$ is relatively quasiconvex if some (equivalently, every) $H$-orbit is relatively quasiconvex in $X$.

Proposition 3.4. Let $G$ be hyperbolic relative to $\mathcal{P}=\left\{P_{1}, \ldots, P_{n}\right\}$, with $P_{i}$ finitely generated. If $G$ acts on a simplicial tree $T$ relative to $\mathcal{P}$ with relatively quasiconvex edge stabilizers, then vertex stabilizers are relatively quasiconvex. 
The proposition applies in particular if edge stabilizers are elementary, since elementary subgroups are relatively quasiconvex. It was proved by Bowditch [5, Proposition 1.2] and Kapovich [41, Lemma 3.5] for $G$ hyperbolic. We generalize Bowditch's argument.

Proof. As usual, we assume that $G$ acts on $T$ minimally and without inversion. Since $G$ is finitely generated, the graph $T / G$ is finite. We also assume that $X$ is a connected graph, and edges of $T$ have length 1 .

Since $P_{i}$ is elliptic in $T$, and stabilizers of points of $X$ are finite, hence elliptic in $T$, there exists an equivariant map $f: X \rightarrow T$ sending vertices to vertices, mapping each edge linearly to an edge path, and constant on each horoball of $\mathcal{B}$.

For each edge $e$ of $T$, let $m_{e}$ be the midpoint of $e$, and $Q_{e}=f^{-1}\left(m_{e}\right)$. Let $v$ be a vertex of $T$, and let $E_{v}$ be the set of edges of $T$ with origin $v$. Let $Q_{v} \subset X$ be the preimage under $f$ of the closed ball of radius $\frac{1}{2}$ around $v$ in $T$. Note that $Q_{e} \subset Q_{v}$ for all $e \in E_{v}$ and $Q_{e} \cap \mathcal{B}=\emptyset$. Also note that $Q_{e} \neq \emptyset$ by minimality of $T$ and connectedness of $X$.

If $f(x)=f(h x)$ for $x \in X$ and $h \in G$, then $h$ fixes $f(x)$. Since $G$ acts cocompactly on $X \backslash \mathcal{B}$, it follows that $G_{v}$ acts cocompactly on $Q_{v} \backslash \mathcal{B}$ and $G_{e}$ acts cocompactly on $Q_{e}=Q_{e} \backslash \mathcal{B}$. In particular, $Q_{e}$ is the $G_{e}$-orbit of a finite set. Relative quasiconvexity of $G_{e}$ implies that $Q_{e}$ is relatively quasiconvex. Since $T / G$ is a finite graph, there exists a common constant $C$ such that all subsets $Q_{e}$ are relatively $C$-quasiconvex.

We now fix a vertex $v$, and we show that $G_{v}$ is relatively quasiconvex. Choose $x \in Q_{v} \backslash \mathcal{B}$. Since $G_{v}$ acts cocompactly on $Q_{v} \backslash \mathcal{B}$, the Hausdorff distance between $Q_{v} \backslash \mathcal{B}$ and the $G_{v}$-orbit of $x$ is finite, so it suffices to prove that $Q_{v}$ is relatively quasiconvex. Let $\gamma$ be a geodesic of $X$ joining two points of $Q_{v}$, and let $\gamma_{0}$ be a maximal subgeodesic contained in $\gamma \backslash Q_{v}$. Considering the image of $\gamma$ in $T$, we see that both endpoints of $\gamma_{0}$ belong to the same $Q_{e}$, for some $e \in E_{v}$. Thus $\gamma_{0} \backslash \mathcal{B}$ is $C$-close to $Q_{e}$, hence to $Q_{v}$. This shows that $Q_{v}$ is relatively $C$-quasiconvex.

A relatively quasiconvex subgroup is relatively hyperbolic in a natural way ([39, Theorem 9.1]). In particular:

Lemma 3.5. If $G_{v}$ is an infinite vertex stabilizer of a tree with finite edge stabilizers, it is hyperbolic relative to the family $\mathcal{P}_{\mid G_{v}}$ of Definition 2.3.

Proof. This follows from Theorem 9.1 of [39], adding finite groups belonging to $\mathcal{P}_{\mid G_{v}}$ to the parabolic structure if needed. 
3.3. The canonical JSJ decomposition. In this section we recall the description of the canonical relative JSJ decomposition. The content of the word canonical is that the JSJ tree (not just the JSJ deformation space) is invariant under automorphisms.

Let $G$ be hyperbolic relative to $\mathcal{P}$, and denote by $\mathcal{A}$ the family of elementary subgroups. In this subsection we fix another (possibly empty) family of finitely generated subgroups $\mathcal{H}=\left\{H_{1}, \ldots, H_{q}\right\}$ and we assume that $G$ is one-ended relative to $\mathcal{P} \cup \mathcal{H}$.

We consider the canonical Out $(G ; \mathcal{P} \cup \mathcal{H})$-invariant JSJ tree $T_{\text {can }}$ defined (under the name $T_{c}^{*}$ ) in Theorem 13.1 of [36] (see also Theorem 7.5 of [34]). It is the tree of cylinders (see Subsection 2.7) of the JSJ deformation space $\mathcal{D}$ over elementary subgroups relative to $\mathcal{P} \cup \mathcal{H}$, and it belongs to $\mathcal{D}$. It is $\operatorname{Out}(G ; \mathcal{P} \cup \mathcal{H})$-invariant because the JSJ deformation space $\mathcal{D}$ is.

Being a tree of cylinders, $T_{\text {can }}$ is bipartite, with vertices $x \in V_{0}\left(T_{\text {can }}\right)$ and $Y \in V_{1}\left(T_{\text {can }}\right)$. Stabilizers of vertices in $V_{0}\left(T_{\text {can }}\right)$ are non-elementary, and stabilizers of vertices in $V_{1}\left(T_{\text {can }}\right)$ are maximal elementary subgroups. Non-elementary vertex stabilizers may be rigid or flexible (see Subsection 2.5), and flexible vertex stabilizers are QH with finite fiber (see Theorem 13.1 of [36]). Elementary vertex stabilizers are infinite by one-endedness, they may be parabolic or loxodromic. Thus there are exactly four possibilities for a vertex $v \in T_{\text {can }}$ :

0 .a. rigid: $G_{v}$ is non-elementary and is elliptic in every $(\mathcal{A}, \mathcal{P} \cup \mathcal{H})$-tree.

0.b. (flexible) $Q H: G_{v}$ is non-elementary and not universally elliptic. Then $v$ is a flexible QH vertex with finite fiber as in Subsection 2.5.

1.a. maximal parabolic: $G_{v}$ is conjugate to a $P_{i}$.

1.b. maximal loxodromic: $G_{v}$ is a maximal virtually cyclic subgroup of $G$, and $G_{v}$ is not parabolic.

Remark 3.6. A QH vertex $v \in V_{0}\left(T_{\text {can }}\right)$ is flexible, except in a few cases; for instance, if $G$ is torsion-free, the only exceptional case is when the underlying surface is a pair of pants (thrice punctured sphere). In these cases we view $v$ as rigid rather than $\mathrm{QH}$. This should not cause confusion. In particular, Propositions 4.1 and 4.7 would remain valid with $v$ viewed as $\mathrm{QH}$.

If $\varepsilon=(x, Y)$ is an edge, then $G_{\varepsilon}=G_{x} \cap G_{Y}$ is an infinite maximal elementary subgroup of $G_{x}$ (but $G_{\varepsilon}$ may fail to be maximal elementary in $G$ and $G_{Y}$ ). In particular, $G_{\varepsilon}$ is always almost malnormal in $G_{x}$, so that Assertion 2 of Lemma 2.12 applies to $T_{\text {can }}$, showing $\mathcal{T}=\operatorname{ker} \rho$. 
Let now $v$ be a $\mathrm{QH}$ vertex. We claim that, if $\mathcal{H}=\emptyset$ and every $P_{i}$ is infinite, then $\mathcal{B}_{v}=\operatorname{Inc}_{v} \cup \mathcal{P}_{\mid G_{v}}$, and $\mathcal{B}_{v}=\operatorname{Inc}_{v}$ if no $P_{i}$ is virtually cyclic (see Definitions 2.1, 2.3 and 2.8).

Groups in Inc $\cos _{v} \cup \mathcal{P}_{\mid G_{v}}$ are infinite maximal elementary subgroups of $G_{v}$, and groups in $\mathcal{B}_{v}$ are virtually cyclic, so $\operatorname{Inc}_{v} \cup \mathcal{P}_{\mid G_{v}} \subset \mathcal{B}_{v}$ by Definition 2.7. Conversely, because of one-endedness, every boundary component of $\Sigma$ is used by $\operatorname{Inc}_{v} \cup \mathcal{P}_{\mid G_{v}}$ [36, Subsection 2.5 and Theorem 13.1]: for any full boundary subgroup $B \in \mathcal{B}_{v}$, there exists a subgroup $H \in \operatorname{Inc}_{v} \cup \mathcal{P}_{\mid G_{v}}$ such that some $G_{v}$-conjugate of $H$ is a finite index subgroup of $B$ (hence equals $B$ ). This proves the converse. Since groups in $\mathcal{B}_{v}$ are virtually cyclic, $\mathcal{B}_{v}=\operatorname{Inc}_{v}$ if no $P_{i}$ is virtually cyclic. This proves the claim.

When $\mathcal{H} \neq \emptyset$, we still have $\operatorname{Inc}_{v} \cup \mathcal{P}_{\mid G_{v}} \subset \mathcal{B}_{v}$. If $H_{j}$ is infinite, the intersection of any of its conjugates with $G_{x}$ is contained in a full boundary subgroup, in particular is virtually cyclic. Conversely, a group of $\mathcal{B}_{v}$ belongs to $\operatorname{Inc}_{v} \cup \mathcal{P}_{\mid G_{v}}$ or contains with finite index a $G_{v}$-conjugate of a group $H \in \mathcal{H}_{\mid G_{v}}$.

This analysis implies that a group $P_{i}$ which is not virtually cyclic is contained in a rigid $G_{x}$ or is equal to some $G_{Y}$ (which may be contained in a rigid $G_{x}$ ). A group $H_{j}$ which is not virtually cyclic is contained in a rigid $G_{x}$ or in a $G_{Y}$.

Lemma 3.7. $T_{\text {can }}$ has finitely generated edge (hence vertex) stabilizers.

We do not assume that the $P_{i}$ 's are slender, so there may exist infinitely generated elementary subgroups.

Proof. Since $G$ is finitely presented relative to $\mathcal{P} \cup \mathcal{H}$, there is an elementary JSJ tree $T_{J}$ relative to $\mathcal{P} \cup \mathcal{H}$ having finitely generated edge stabilizers ([35], Theorem 5.1). The tree $T_{\text {can }}$ is the tree of cylinders of $T_{J}$.

Consider an edge $\varepsilon=(x, Y)$ of $T_{\text {can }}$, and $G_{\varepsilon}=G_{x} \cap G_{Y}$. We view $Y$ as a subtree of $T_{J}$ containing $x$. If $G_{Y}$ is virtually cyclic, then $G_{\varepsilon}$ is obviously finitely generated, so we can assume that $G_{Y}$ is a maximal parabolic group $P_{i}$.

Since $G_{Y}=P_{i}$ is elliptic in $T_{J}$ and leaves $Y$ invariant, it fixes a vertex $y \in Y$. If $y=x$, then $G_{Y} \subset G_{x}$, so $G_{\varepsilon}=G_{Y}=P_{i}$ is finitely generated. If $y \neq x$, let $e$ be the initial edge of the segment $[x, y]$ in $T_{J}$. It is contained in $Y$, so $G_{e} \subset G_{Y}$, hence $G_{e} \subset G_{x} \cap G_{Y} \subset G_{x} \cap G_{y} \subset G_{e}$. It follows that $G_{\varepsilon}=G_{x} \cap G_{Y}=G_{e}$ is finitely generated.

By Proposition 3.4, vertex groups of $T_{\text {can }}$ are relatively quasiconvex, hence relatively hyperbolic. We make the parabolic structure explicit. 
Lemma 3.8. If $x \in V_{0}\left(T_{\mathrm{can}}\right)$, the group $G_{x}$ is hyperbolic relative to the finite family of finitely generated subgroups $Q_{x}=\operatorname{Inc}_{x} \cup \mathcal{P}_{\mid G_{x}}$, where $\operatorname{Inc}_{x}$ is a set of representatives of conjugacy classes of incident edge stabilizers and $\mathcal{P}_{\mid G_{x}}$ is the induced structure (see Definitions 2.1 and 2.3).

Proof. By Theorem 9.1 of [39], we have to consider infinite groups of the form $G_{x} \cap g P_{i} g^{-1}$. Recall that stabilizers of vertices adjacent to $x$ are maximal elementary subgroups, and distinct maximal elementary subgroups have finite intersection. Thus $g P_{i} g^{-1}$ must be the stabilizer of an adjacent vertex, or have $x$ as unique fixed point, so $G_{x} \cap g P_{i} g^{-1}$ is conjugate in $G_{x}$ to a group in $\operatorname{Inc}_{x} \cup \mathcal{P}_{\mid G_{x}}$. Conversely, a group in $\operatorname{Inc}_{x} \cup \mathcal{P}_{\mid G_{x}}$ which is not an infinite group of the form $G_{x} \cap g P_{i} g^{-1}$ is finite or is a loxodromic maximal virtually cyclic subgroup of $G_{x}$. Such groups may be added to the parabolic structure.

\subsection{Rigid groups have finitely many automorphisms.}

Theorem 3.9. Let $G$ be hyperbolic relative to finitely generated subgroups $\mathcal{P}=$ $\left\{P_{1}, \ldots, P_{n}\right\}$, with $P_{i} \neq G$. Let $\mathcal{H}=\left\{H_{1}, \ldots, H_{q}\right\}$ be another family of finitely generated subgroups. If $\operatorname{Out}\left(G ; \mathcal{P}, \mathcal{H}^{(\mathrm{t})}\right)$ is infinite, then $G$ splits over an elementary subgroup relative to $\mathcal{P} \cup \mathcal{H}$.

The hypothesis means that there are infinitely many (classes of) automorphisms which map each $P_{i}$ to a conjugate (in an arbitrary way) and act on each $H_{j}$ as conjugation by an element of $G$.

The proof of the theorem has two steps. First, using the Bestvina-Paulin method (see [54]), extended by Belegradek-Szczepański [1] to relatively hyperbolic groups, one constructs an action of $G$ on an R-tree $T$. Rips theory then yields a splitting. This is fairly standard but there are technical difficulties, in particular because the action on $T$ is not necessarily stable if the $P_{i}$ 's are not assumed to be slender. Details are in Section 9.

\section{Automorphisms of one-ended relatively hyperbolic groups}

Let $G$ be hyperbolic relative to $\mathcal{P}=\left\{P_{1}, \ldots, P_{n}\right\}$, with $P_{i}$ infinite and finitely generated. We assume that $G$ is one-ended relative to $\mathcal{P}$. In Subsection 4.1 we study $\operatorname{Out}(G ; \mathcal{P})$ through its action on the canonical JSJ tree. This leads to the main results of this section, which are stated in Subsection 4.2. In Subsection 4.3 we study automorphisms which act trivially on another family $\mathcal{H}$. 
4.1. Automorphisms of the canonical JSJ splitting. Let $T_{\text {can }}$ be the canonical $\operatorname{Out}(G ; \mathcal{P})$-invariant JSJ tree as in Subsection 3.3 (with $\mathcal{H}=\emptyset$ ), and $\Gamma_{\text {can }}=$ $T_{\text {can }} / G$. Edge stabilizers are infinite elementary subgroups. Vertex stabilizers may be rigid (non-elementary), QH with finite fiber, maximal parabolic (conjugate to a $P_{i}$ ), or maximal loxodromic (virtually cyclic). A rigid or $\mathrm{QH}$ stabilizer $G_{x}$ fixes a unique point in $T_{\text {can }}$, hence is equal to its normalizer; incident edge stabilizers are maximal elementary subgroups of $G_{x}$.

We study $\operatorname{Out}(G ; \mathcal{P})$ through its action on $T_{\text {can }}$ as in Subsection 2.6. In general, $\operatorname{Out}(G ; \mathcal{P})$ is a proper subgroup of $\operatorname{Out}\left(T_{\text {can }}\right)$.

We define finite index subgroups $\operatorname{Out}^{0}(G ; \mathcal{P})$ and $\operatorname{Out}^{0}\left(G ; \mathcal{P}^{(t)}\right)$ by taking the intersection of $\operatorname{Out}(G ; \mathcal{P})$ and $\operatorname{Out}\left(G ; \mathcal{P}^{(\mathrm{t})}\right)$ with the group $\operatorname{Out}^{\mathrm{0}}\left(T_{\text {can }}\right)$ consisting of automorphisms acting trivially on the graph $\Gamma_{\text {can }}=T_{\text {can }} / G$.

By the second assertion of Lemma 2.12, the kernel of

$$
\rho: \operatorname{Out}^{0}\left(T_{\text {can }}\right) \longrightarrow \prod_{v \in V} \operatorname{Out}\left(G_{v}\right)
$$

is the group of twists $\mathcal{T}$. Note that $\mathcal{T} \subset$ Out $^{0}\left(G ; \mathcal{P}^{(\mathrm{t})}\right) \subset \operatorname{Out}^{0}(G ; \mathcal{P})$ since every $P_{i}$ is elliptic in $T_{\text {can }}$ and a twist acts as a conjugation on any vertex stabilizer.

The group $\mathcal{T}$ is the image in $\operatorname{Out}(G)$ of a finite direct product $\prod_{e \in E} Z_{G_{o(e)}}\left(G_{e}\right)$. Each factor is virtually cyclic or contained in a conjugate of some $P_{i}$.

We now consider the image of $\operatorname{Out}^{0}(G ; \mathcal{P})$ and $\operatorname{Out}^{0}\left(G ; \mathcal{P}^{(t)}\right)$ by

$$
\rho_{v}: \operatorname{Out}^{0}\left(T_{\text {can }}\right) \longrightarrow \operatorname{Out}\left(G_{v}\right),
$$

for $v$ a vertex of $\Gamma_{\text {can }}$ (viewed as a vertex of $T_{\text {can }}$ with stabilizer $G_{v}$ ). Using Theorem 3.9, we shall show that both images are finite if $G_{v}$ is rigid. If $G_{v}=P_{i}$, the index of $\operatorname{Out}\left(G_{v} ; \operatorname{Inc}_{v}^{(\mathrm{t})}\right)$ in the image of $\operatorname{Out}^{0}(G ; \mathcal{P})$ is finite because $w$ is rigid whenever $e=v w$ is an edge with $\operatorname{Out}\left(G_{e}\right)$ infinite. More precisely:

Proposition 4.1. The images of $\operatorname{Out}^{0}(G ; \mathcal{P})$ and $\operatorname{Out}^{0}\left(G ; \mathcal{P}^{(\mathrm{t})}\right)$ by

$$
\rho_{v}: \operatorname{Out}^{0}\left(T_{\text {can }}\right) \longrightarrow \operatorname{Out}\left(G_{v}\right)
$$

may be described as follows:

- if $G_{v}$ is virtually cyclic or rigid, both images are finite;

- if $G_{v}$ is a QH vertex stabilizer, both images contain $\operatorname{Out}\left(G_{v} ; \mathcal{B}_{v}^{(\mathrm{t})}\right)$ with finite index;

- if $G_{v}$ is (conjugate to) $P_{i}$, the image of $\operatorname{Out}^{0}\left(G ; \mathcal{P}^{(\mathrm{t})}\right)$ is trivial. The image of $\operatorname{Out}^{0}(G ; \mathcal{P})$ contains $\operatorname{Out}\left(G_{v} ; \operatorname{Inc}_{v}^{(\mathrm{t})}\right)$ with finite index. 
If $e$ is any edge of $T_{\text {can }}$, the images of $\operatorname{Out}^{0}(G ; \mathcal{P})$ and $\operatorname{Out}^{0}\left(G ; \mathcal{P}^{(\mathrm{t})}\right)$ by

$$
\rho_{e}: \operatorname{Out}^{0}\left(T_{\text {can }}\right) \longrightarrow \operatorname{Out}\left(G_{e}\right)
$$

are finite.

See Definition 2.8 for the definition of the family of full boundary subgroups $\mathcal{B}_{v}$, and recall that $\mathcal{B}_{v}=\operatorname{Inc}_{v}$ if no $P_{i}$ is virtually cyclic.

Proof. If $G_{v}$ is virtually cyclic, $\operatorname{Out}\left(G_{v}\right)$ is finite. If $G_{v}$ is rigid, finiteness follows from Theorem 3.9, as we now explain. We have seen (Lemma 3.8) that $G_{v}$ is hyperbolic relative to a finite family $Q_{v}=\operatorname{Inc}_{v} \cup \mathcal{P}_{\mid G_{v}}$ consisting of incident edge groups and conjugates of the $P_{i}$ 's having $v$ as unique fixed point. These groups are finitely generated by Lemma 3.7. By Lemma 2.10, the group $\rho_{v}\left(\operatorname{Out}^{0}(G ; \mathcal{P})\right)$ is contained in $\operatorname{Out}\left(G_{v} ; Q_{v}\right)$. If it is infinite, $G_{v}$ splits over an elementary subgroup relative to $Q_{v}$ by Theorem 3.9 (applied with $\mathcal{P}=Q_{v}$ and $\mathcal{H}=\emptyset$ ). By Lemma 2.6, this splitting may be used to refine $T_{\text {can }}$, yielding an elementary splitting of $G$ relative to $\mathcal{P}$ in which $G_{v}$ is not elliptic. This contradicts rigidity.

If $G_{v}$ is $\mathrm{QH}$, first note that

$$
\operatorname{Out}\left(G_{v} ; \mathcal{B}_{v}^{(\mathrm{t})}\right)=\operatorname{Out}\left(G_{v} ; \operatorname{Inc}_{v}^{(\mathrm{t})}, \mathcal{P}_{\mid G_{v}}^{(\mathrm{t})}\right)
$$

and

$$
\operatorname{Out}\left(G_{v} ; \mathcal{B}_{v}\right)=\operatorname{Out}\left(G_{v} ; \operatorname{Inc}_{v}, \mathcal{P}_{\mid G_{v}}\right)
$$

because $\mathcal{B}_{v}=\operatorname{Inc}_{v} \cup \mathcal{P}_{\mid G_{v}}$ (see Subsection 3.3, recalling that groups in $\mathcal{P}$ are assumed to be infinite).

Lemma 2.10 then yields

$$
\operatorname{Out}\left(G_{v} ; \mathcal{B}_{v}^{(\mathrm{t})}\right) \subset \rho_{v}\left(\operatorname{Out}^{0}\left(G ; \mathcal{P}^{(\mathrm{t})}\right)\right) \subset \rho_{v}\left(\operatorname{Out}^{0}(G ; \mathcal{P})\right) \subset \operatorname{Out}\left(G_{v} ; \mathcal{B}_{v}\right) .
$$

We conclude by observing that $\operatorname{Out}\left(G_{v} ; \mathcal{B}_{v}^{(\mathrm{t})}\right)$ has finite index in $\operatorname{Out}\left(G_{v} ; \mathcal{B}_{v}\right)$ because groups in $\mathcal{B}_{v}$ are virtually cyclic, hence have finite outer automorphism group.

If $G_{v}$ is (conjugate to) $P_{i}$, the image of $\operatorname{Out}^{0}\left(G ; \mathcal{P}^{(\mathrm{t})}\right)$ is clearly trivial. Since $\mathcal{P}_{\mid G_{v}}$ equals $\left\{G_{v}\right\}$ or is empty, the image of $\operatorname{Out}^{0}(G ; \mathcal{P})$ contains $\operatorname{Out}\left(G_{v} ; \operatorname{Inc}_{v}^{(\mathrm{t})}\right)=$ $\operatorname{Out}\left(G_{v} ; \operatorname{Inc}_{v}^{(\mathrm{t})}, \mathcal{P}_{\mid G_{v}}\right)$, and we have to show that the index is finite. If $\operatorname{Out}\left(G_{\varepsilon}\right)$ is finite for every incident edge $\varepsilon$, this follows from Lemma 2.10. In general, we have to control the action of automorphisms on $G_{\varepsilon}$ for incident edges $\varepsilon$ with $\operatorname{Out}\left(G_{\varepsilon}\right)$ infinite. Note that there is no natural map from $\operatorname{Out}\left(G_{v} ; \operatorname{Inc}_{v}\right)$ to $\operatorname{Out}\left(G_{\varepsilon}\right)$ if $N_{G_{v}}\left(G_{\varepsilon}\right)$ acts non-trivially on $G_{\varepsilon}$. 
Infiniteness of $\operatorname{Out}\left(G_{\varepsilon}\right)$ implies that the other endpoint of $\varepsilon$ is a rigid vertex $x$ : it cannot be $\mathrm{QH}$ since $G_{\varepsilon}$ would then be virtually cyclic. As explained above, the image of $\operatorname{Out}^{0}(G ; \mathcal{P})$ in $\operatorname{Out}\left(G_{x}\right)$ is finite. Any $\Phi \in \operatorname{Out}^{0}(G ; \mathcal{P})$ has a representative $\alpha$ leaving $G_{x}$ and $G_{v}$ invariant (the associated map $f_{\alpha}$ fixes $\varepsilon$ ). Replacing $\operatorname{Out}^{0}(G ; \mathcal{P})$ by a finite index subgroup, we may suppose that $\alpha$ acts on $G_{x}$ as conjugation by some element $g$. This $g$ must be in $G_{x}$ since $G_{x}$ equals its normalizer, and in fact in $G_{\varepsilon}$ because $G_{\varepsilon}$ is almost malnormal in $G_{x}$. This shows that $\Phi$ maps into $\operatorname{Out}\left(G_{v} ; G_{\varepsilon}^{(\mathrm{t})}\right)$. Arguing in this way for each incident edge proves the result for the image of $\rho_{v}$.

Since any edge of $T_{\text {can }}$ has a vertex $v$ with $G_{v}$ virtually cyclic or conjugate to a $P_{i}$, the previous argument also shows finiteness for images by $\rho_{e}$.

Definition 4.2. Let $\Sigma$ be a compact 2-dimensional hyperbolic orbifold. The extended mapping class group $M C G^{*}(\Sigma)$ is the group of outer automorphisms of $\pi_{1}(\Sigma)$ preserving the set of boundary subgroups.

If $v$ is a $\mathrm{QH}$ vertex of $T_{\text {can }}$ with underlying orbifold $\Sigma$ and finite fiber $F$, we define $M C G_{T_{\text {can }}}^{0}(\Sigma)$ as the group $\operatorname{Out}\left(G_{v} ; \mathcal{B}_{v}^{(\mathrm{t})}\right)$. This group maps with finite kernel onto a finite index subgroup of $M C G^{*}(\Sigma)$ [17, pp. 240 and 268].

As noted in [26, 17], one can understand the extended mapping class group of an orbifold with mirrors in terms of the extended mapping class group of a suborbifold without mirrors.

If $G$ is torsion free, $\Sigma$ is a surface, $M C G^{*}(\Sigma)$ is the group of isotopy classes of homeomorphisms, and $M C G_{T_{\text {can }}}^{0}(\Sigma)$ is the group of isotopy classes of homeomorphisms which map each boundary component to itself in an orientation-preserving way (in this case it only depends on $\Sigma$ since the fiber is trivial).

Mapping class groups are usually infinite, but there are exceptions. In the torsion-free case, the exceptions are the pair of pants and the twice punctured projective plane [44, Cor 4.6]; all other hyperbolic surfaces contain an essential 2 -sided simple closed curve not bounding a Möbius band, so there is a Dehn twist of infinite order. As a $\mathrm{QH}$ vertex, a pair of pants is rigid; every simple closed curve is homotopically trivial or boundary parallel. A twice punctured projective plane is flexible, but every 2-sided simple closed curve is homotopically trivial, boundary parallel, or bounds a Möbius band, so there is no non-trivial Dehn twist.

4.2. Automorphisms of $\boldsymbol{G}$. Motivated by the previous subsection, we define a subgroup $\operatorname{Out}^{1}\left(G ; \mathcal{P}^{(\mathrm{t})}\right) \subset \operatorname{Out}^{0}\left(G ; \mathcal{P}^{(\mathrm{t})}\right)$ as the set of $\Phi$ such that $\rho_{v}(\Phi)$ is trivial if $G_{v}$ is virtually cyclic, rigid, or conjugate to a $P_{i}$, and

$$
\rho_{v}(\Phi) \in \operatorname{Out}\left(G_{v} ; \mathcal{B}_{v}^{(\mathrm{t})}\right)=M C G_{T_{\text {can }}}^{0}(\Sigma)
$$


if $G_{v}$ is $\mathrm{QH}$. Proposition 4.1 shows that this subgroup has finite index. We define a finite index subgroup $\operatorname{Out}^{1}(G ; \mathcal{P}) \subset \operatorname{Out}^{0}(G ; \mathcal{P})$ similarly, allowing $\rho_{v}(\Phi) \in$ $\operatorname{Out}\left(G_{v} ; \operatorname{Inc}_{v}^{(\mathrm{t})}\right)$ if $G_{v}$ is conjugate to a $P_{i}$.

We may now sum up the discussion in Subsection 4.1 as:

Theorem 4.3. Let $G$ be hyperbolic relative to $\mathcal{P}=\left\{P_{1}, \ldots, P_{n}\right\}$, with $P_{i}$ infinite and finitely generated. Assume that $G$ is one-ended relative to $\mathcal{P}$.

Then $\operatorname{Out}\left(G ; \mathcal{P}^{(\mathrm{t})}\right)$ and $\operatorname{Out}(G ; \mathcal{P})$ have finite index subgroups $\operatorname{Out}^{1}\left(G ; \mathcal{P}^{(\mathrm{t})}\right)$ and Out $^{1}(G ; \mathcal{P})$ which fit in exact sequences

$$
1 \longrightarrow \mathcal{T} \longrightarrow \operatorname{Out}^{1}\left(G ; \mathcal{P}^{(\mathrm{t})}\right) \longrightarrow \prod_{i=1}^{p} \operatorname{MCG}_{T_{\text {can }}}^{0}\left(\Sigma_{i}\right) \longrightarrow 1
$$

and

$$
1 \longrightarrow \mathcal{T} \longrightarrow \operatorname{Out}^{1}(G ; \mathcal{P}) \longrightarrow \prod_{i=1}^{p} M C G_{T_{\text {can }}}^{0}\left(\Sigma_{i}\right) \times \prod_{j} \operatorname{Out}\left(P_{j} ; \operatorname{Inc}_{P_{j}}^{(\mathrm{t})}\right) \longrightarrow 1
$$

where:

- $\mathcal{T}$ is the group of twists of the canonical elementary JSJ decomposition $T_{\text {can }}$ relative to $\mathcal{P}$; it is a quotient of a finite direct product where each factor is a subgroup of $G$ which is virtually cyclic or contained in a $P_{i}$;

- $\Sigma_{1}, \ldots, \Sigma_{p}$ are the 2-orbifolds occuring in flexible $Q H$ vertices $v$ of $T_{\text {can }}$, and $M C G_{T_{\mathrm{can}}}^{0}\left(\Sigma_{i}\right)$ maps with finite kernel onto a finite index subgroup of the extended mapping class group $M C G^{*}\left(\Sigma_{i}\right)$;

- the last product is taken only over those $P_{j}$ 's which occur as vertex stabilizers of $T_{\mathrm{can}}$, and $\operatorname{Inc}_{P_{j}}$ is the set of incident edge groups.

Note that $\mathcal{T}$ is slender (resp. small, virtually solvable, virtually nilpotent, virtually abelian) if the $P_{i}$ 's are. Also note that $\operatorname{Out}(G ; \mathcal{P})$ has finite index in $\operatorname{Out}(G)$ if the $P_{i}$ 's are small but not virtually cyclic, since they may be characterized up to conjugacy as maximal among the subgroups of $G$ which are small but not virtually cyclic. More generally, this holds if no $P_{i}$ is relatively hyperbolic [51, Lemma 3.2].

Recall that $G$ is toral relatively hyperbolic if it is torsion-free and hyperbolic relative to a finite family $\mathcal{P}$ of finitely generated abelian subgroups. Limit groups, and more generally groups acting freely on $\mathbb{R}^{n}$-trees, are toral relatively hyperbolic $[15,30]$. 
Corollary 4.4. Let $G$ be toral relatively hyperbolic and one-ended. Then some finite index subgroup $\operatorname{Out}^{1}(G)$ of $\operatorname{Out}(G)$ fits in an exact sequence

$$
1 \longrightarrow \mathcal{T} \longrightarrow \operatorname{Out}^{1}(G) \longrightarrow \prod_{i=1}^{p} \operatorname{MCG}^{0}\left(\Sigma_{i}\right) \times \prod_{k=1}^{m} \mathrm{GL}_{r_{k}, n_{k}}(\mathbb{Z}) \longrightarrow 1
$$

where $\mathcal{T}$ is finitely generated free abelian, $M C G^{0}\left(\Sigma_{i}\right)$ is the group of isotopy classes of homeomorphisms of a compact surface $\Sigma_{i}$ mapping each boundary component to itself in an orientation-preserving way, and

$$
\mathrm{GL}_{r, n}(\mathbb{Z})=M_{r, n}(\mathbb{Z}) \rtimes \mathrm{GL}_{r}(\mathbb{Z})
$$

is the group of automorphisms of $\mathbb{Z}^{n+r}$ fixing the first $n$ generators.

See Theorem 5.3 of [9] and Theorem 6.5 of [33] for the case of limit groups, based on results from [43] and [9].

Proof. We may assume that no $P_{i} \in \mathcal{P}$ is cyclic, so $\operatorname{Out}(G ; \mathcal{P})$ has finite index in $\operatorname{Out}(G)$. If $P_{j}$ is isomorphic to $\mathbb{Z}^{a}$, then $\operatorname{Out}\left(P_{j} ; \operatorname{Inc}_{P_{j}}^{(\mathrm{t})}\right)$ is isomorphic to some $\mathrm{GL}_{r, n}(\mathbb{Z})$ with $r+n=a$, so the exact sequence follows from Theorem 4.3. We know that the group of twists $\mathcal{T}$ of $T_{\text {can }}$ is finitely generated and abelian. There remains to check that it is torsion-free.

Recall from Subsection 2.6 that $\mathcal{T}$ is generated by the product of all $Z_{G_{o(e)}}\left(G_{e}\right)$, subject to edge and vertex relations. Denoting an edge of $\Gamma_{\text {can }}=T_{\text {can }} / G$ by $\varepsilon=(x, Y)$ with $x \in V_{0}\left(\Gamma_{\text {can }}\right)$ and $Y \in V_{1}\left(\Gamma_{\text {can }}\right)$, there is no relation at the vertex $x$ since $Z\left(G_{x}\right)$ is trivial. Moreover, $Z_{G_{x}}\left(G_{\varepsilon}\right)=G_{\varepsilon}$, so the edge relation identifies the twists around $\varepsilon$ near $x$ with twists near $Y$. Thus $\mathcal{T}$ is the direct product, over vertices $v$ of $\Gamma_{\text {can }}$ carrying an abelian group, of $\left(\prod_{e \in E_{v}} Z_{G_{v}}\left(G_{e}\right)\right) / Z\left(G_{v}\right)$ where $E_{v}$ is the set of oriented edges with origin $v$ and $Z\left(G_{v}\right)$ is embedded diagonally. Since $G_{v}$ is abelian, $Z_{G_{v}}\left(G_{e}\right)=Z\left(G_{v}\right)=G_{v}$, so $\mathcal{T}$ is isomorphic to a finite direct product $\prod\left(G_{v}\right)^{\left|E_{v}\right|-1}$ of abelian vertex groups. It is therefore torsion-free.

Corollary 4.5. If $G$ is a toral relatively hyperbolic group, $\operatorname{Out}(G)$ is virtually torsion-free and has a finite index subgroup with a finite classifying space.

Proof. This follows from Corollary 4.4 if $G$ is one-ended. In general, we write $G=G_{1} * \cdots * G_{q} * F$ with $G_{\ell}$ one-ended and $F$ free. All groups $G_{\ell}$ and $G_{\ell} / Z\left(G_{\ell}\right)$ have a finite classifying space [14], so we can apply Theorem 5.2 of [33]. (We mention here that the arguments given in [33] are insufficient to get a finite classifying space: there should exist finite classifying spaces for the groups $\operatorname{Out}^{S}(G)$ themselves (rather than for finite index subgroups); this is achieved by restricting to some finite index subgroup of $\operatorname{Out}(G)$, see [37] for details.) 
4.3. The relative case. We generalize the analysis of Subsection 4.1 to a relative situation.

Let $G, \mathcal{P}$ be as above, and fix another family of finitely generated subgroups $\mathcal{H}=\left\{H_{1}, \ldots, H_{q}\right\}$. Assume that $G$ is one-ended relative to $\mathcal{P} \cup \mathcal{H}$, and let now $T_{\text {can }}$ be the canonical elementary JSJ tree relative to $\mathcal{P} \cup \mathcal{H}$.

Theorem 4.6. Under these hypotheses, both $\operatorname{Out}\left(G ; \mathcal{P}^{(\mathrm{t})}, \mathcal{H}^{(\mathrm{t})}\right)$ and $\operatorname{Out}\left(G ; \mathcal{P}, \mathcal{H}^{(\mathrm{t})}\right)$ have finite index subgroups $\operatorname{Out}^{1}\left(G ; \mathcal{P}^{(\mathrm{t})}, \mathcal{H}^{(\mathrm{t})}\right)$ and $\operatorname{Out}^{1}\left(G ; \mathcal{P}, \mathcal{H}^{(\mathrm{t})}\right)$ which fit in exact sequences

$$
1 \longrightarrow \mathcal{T} \longrightarrow \operatorname{Out}^{1}\left(G ; \mathcal{P}^{(\mathrm{t})}, \mathcal{H}^{(\mathrm{t})}\right) \longrightarrow \prod_{i=1}^{p} M C G_{T_{\mathrm{can}}}^{1}\left(\Sigma_{i}\right) \longrightarrow 1
$$

and

$$
\begin{aligned}
1 \longrightarrow \mathcal{T} \longrightarrow \operatorname{Out}^{1}\left(G ; \mathcal{P}, \mathcal{H}^{(\mathrm{t})}\right) & \\
& \longrightarrow \prod_{i=1}^{p} M C G_{T_{\mathrm{can}}}^{1}\left(\Sigma_{i}\right) \times \prod_{j} \operatorname{Out}\left(P_{j} ; \operatorname{Inc}_{P_{j}}^{(\mathrm{t})}, \mathcal{H}_{\mid P_{j}}^{(\mathrm{t})}\right) \longrightarrow 1,
\end{aligned}
$$

as in Theorem 4.3.

The group $M C G_{T_{\text {can }}}^{1}\left(\Sigma_{i}\right)$ equals $\operatorname{Out}\left(G_{v} ; \mathcal{B}_{v}^{(\mathrm{t})}, \mathcal{F}_{v}^{(\mathrm{t})}\right)$, where $\mathcal{F}_{v}$ is a set of representatives of conjugacy classes of finite subgroups in $G_{v}$; it is a finite index subgroup of $M C G_{T_{\text {can }}}^{0}\left(\Sigma_{i}\right)=\operatorname{Out}\left(G_{v} ; \mathcal{B}_{v}^{(\mathrm{t})}\right)$.

Note that $\mathcal{F}_{v}$ is a finite set since the $\mathrm{QH}$ vertex group $G_{v}$ maps onto a 2-orbifold group with finite kernel. The family $\mathcal{F}_{v}$ is not needed if all groups in $\mathcal{H}$ are infinite (see the proof below).

The theorem is proved as in the absolute case, replacing Proposition 4.1 by the following result.

Proposition 4.7. The images of $\operatorname{Out}^{0}\left(G ; \mathcal{P}, \mathcal{H}^{(\mathrm{t})}\right)$ and $\operatorname{Out}^{0}\left(G ; \mathcal{P}^{(\mathrm{t})}, \mathcal{H}^{(\mathrm{t})}\right)$ by

$$
\rho_{v}: \operatorname{Out}^{0}\left(T_{\text {can }}\right) \longrightarrow \operatorname{Out}\left(G_{v}\right)
$$

may be described as follows;

- if $G_{v}$ is virtually cyclic or rigid, both images are finite:

- if $G_{v}$ is a $Q H$ vertex stabilizer, both images contain $\operatorname{Out}\left(G_{v} ; \mathcal{B}_{v}^{(\mathrm{t})}, \mathcal{F}_{v}^{(\mathrm{t})}\right)$ with finite index (where $\mathcal{F}_{v}$ is as in Theorem 4.6 and $\mathcal{B}_{v}$ is as in Definition 2.8);

- if $G_{v}$ is (conjugate to) $P_{i}$, the image of $\operatorname{Out}^{0}\left(G ; \mathcal{P}^{(\mathrm{t})}, \mathcal{H}^{(\mathrm{t})}\right)$ is trivial. The image of $\operatorname{Out}^{0}\left(G ; \mathcal{P}, \mathcal{H}^{(\mathrm{t})}\right)$ contains $\operatorname{Out}\left(G_{v} ; \operatorname{Inc}_{v}^{(\mathrm{t})}, \mathcal{H}_{\mid G_{v}}^{(\mathrm{t})}\right)$ with finite index. 
Proof. We only mention the differences with the proof of Proposition 4.1.

If $v$ is a (non-elementary) rigid vertex, the images of $\operatorname{Out}^{0}\left(G ; \mathcal{P}, \mathcal{H}^{(\mathrm{t})}\right)$ and $\operatorname{Out}^{0}\left(G ; \mathcal{P}^{(\mathrm{t})}, \mathcal{H}^{(\mathrm{t})}\right)$ by $\rho_{v}$ are contained in $\operatorname{Out}\left(G_{v} ; Q_{v}, \mathcal{H}_{\mid G_{v}}^{(\mathrm{t})}\right)$ by Lemma 2.10. It follows that the images are finite since, otherwise, Theorem 3.9 would yield a splitting relative to $Q_{v} \cup \mathcal{H}_{\mid G_{v}}$, which extends to a splitting of $G$ relative to $\mathcal{P} \cup \mathcal{H}$ by Lemma 2.6 .

When $G_{v}$ is conjugate to a parabolic group $P_{j}$, Lemma 2.10 says that the image of $\operatorname{Out}^{0}\left(G ; \mathcal{P}, \mathcal{H}^{(\mathrm{t})}\right)$ contains $\operatorname{Out}\left(G_{v} ; \operatorname{Inc}_{v}^{(\mathrm{t})}, \mathcal{H}_{\mid G_{v}}^{(\mathrm{t})}\right)$, and the index is finite for the same reason as before.

When $G_{v}$ is $\mathrm{QH}$, we write

$$
\begin{aligned}
\operatorname{Out}\left(G_{v} ; \operatorname{Inc}_{v}^{(\mathrm{t})}, \mathcal{P}_{\mid G_{v}}, \mathcal{H}_{\mid G_{v}}^{(\mathrm{t})}\right) & \subset \rho_{v}\left(\operatorname{Out}^{\mathrm{0}}\left(T ; \mathcal{P}, \mathcal{H}^{(\mathrm{t})}\right)\right) \\
& \subset \operatorname{Out}\left(G_{v} ; \operatorname{Inc}_{v}, \mathcal{P}_{\mid G_{v}}, \mathcal{H}_{\mid G_{v}}^{(\mathrm{t})}\right)
\end{aligned}
$$

using Lemma 2.10.

The proof in the non-relative case relied on the equality

$$
\mathcal{B}_{v}=\operatorname{Inc}_{v} \cup \mathcal{P}_{\mid G_{v}} .
$$

Here (see Subsection 3.3) we have $\operatorname{Inc}_{v} \cup \mathcal{P}_{\mid G_{v}} \subset \mathcal{B}_{v}$, and a group $B \in \mathcal{B}_{v}$ not in $\operatorname{Inc}_{v} \cup \mathcal{P}_{\mid G_{v}}$ contains with finite index a group $H^{\prime}$ conjugate to some $H \in$ $\mathcal{H}_{\mid G_{v}}$. Since $B$ is the only maximal elementary subgroup of $G_{v}$ containing $H^{\prime}$, any automorphism preserving $H^{\prime}$ preserves $B$, so

$$
\operatorname{Out}\left(G_{v} ; \operatorname{Inc}_{v}, \mathcal{P}_{\mid G_{v}}, \mathcal{H}_{\mid G_{v}}^{(\mathrm{t})}\right) \subset \operatorname{Out}\left(G_{v} ; \mathcal{B}_{v}\right) .
$$

If all groups in $\mathcal{H}$ are infinite, the intersection of any conjugate of $H_{j}$ with $G_{v}$ is contained in a full boundary subgroup, so

$$
\operatorname{Out}\left(G_{v} ; \mathcal{B}_{v}^{(\mathrm{t})}\right) \subset \operatorname{Out}\left(G_{v} ; \operatorname{Inc}_{v}^{(\mathrm{t})}, \mathcal{P}_{\mid G_{v}}, \mathcal{H}_{\mid G_{v}}^{(\mathrm{t})}\right) .
$$

Otherwise $\mathcal{H}_{\mid G_{v}}$ may contain finite groups (fixing $v$ but no other vertex of $T_{\text {can }}$ ) and we can only write

$$
\operatorname{Out}\left(G_{v} ; \mathcal{B}_{v}^{(\mathrm{t})}, \mathcal{F}_{v}^{(\mathrm{t})}\right) \subset \operatorname{Out}\left(G_{v} ; \operatorname{Inc}_{v}^{(\mathrm{t})}, \mathcal{P}_{\mid G_{v}}, \mathcal{H}_{\mid G_{v}}^{(\mathrm{t})}\right) .
$$

The proposition follows because the index of $\operatorname{Out}\left(G_{v} ; \mathcal{B}_{v}^{(\mathrm{t})}, \mathcal{F}_{v}^{(\mathrm{t})}\right)$ in $\operatorname{Out}\left(G_{v} ; \mathcal{B}_{v}\right)$ is finite.

Remark 4.8. Because we use Theorem 3.9 to control automorphisms of rigid groups, we do not have a similar result concerning $\operatorname{Out}\left(G ; \mathcal{P}^{(t)} \cup \mathcal{H}\right)$ or $\operatorname{Out}(G ; \mathcal{P} \cup$ $\mathcal{H})$ : we have to impose that automorphisms act trivially on $\mathcal{H}$. We also need finite generation of groups in $\mathcal{H}$. 
Arguing as in the previous subsection, one gets:

Corollary 4.9. Let $G$ be toral relatively hyperbolic, one-ended relative to a family $\mathcal{H}=\left\{H_{1}, \ldots, H_{q}\right\}$ of finitely generated subgroups. Then $\operatorname{Out}\left(G ; \mathcal{H}^{(\mathrm{t})}\right)$ has a finite index subgroup $\operatorname{Out}^{1}\left(G ; \mathcal{H}^{(\mathrm{t})}\right)$ fitting in an exact sequence as in Corollary 4.4.

\section{The modular group}

The goal of this section is to show that the modular group, usually defined by considering all suitable splittings of a group $G$, may be seen on a single splitting, namely the canonical JSJ decomposition.

5.1. Definitions and examples. Let $G$ be hyperbolic relative to $\mathcal{P}=\left\{P_{1}, \ldots, P_{n}\right\}$, where each $P_{i}$ is finitely generated. Without loss of generality, we assume that no $P_{i}$ is virtually cyclic (in particular, $P_{i}$ is infinite). Let $\mathcal{H}$ be another finite family of finitely generated subgroups $H_{j}$ such that every $P_{i}$ which contains a free group $F_{2}$ is contained in a group of $\mathcal{H}$.

In particular, we may take $\mathcal{H}=\mathcal{P}$, or $\mathcal{H}=\emptyset$ if every $P_{i}$ is small. We will assume that $G$ is one-ended relative to $\mathcal{H}$ (equivalently, relative to $\mathcal{P} \cup \mathcal{H}$ since every $P_{i}$ is one-ended or contained in a group of $\mathcal{H}$ ).

We consider trees $T$ with elementary edge stabilizers, which are relative to $\mathcal{H}$ (universal ellipticity will be with respect to these trees, unless indicated otherwise). They are not necessarily relative to $P_{i}$ if $P_{i}$ is small, but our assumption on $\mathcal{H}$ implies that elementary subgroups which are not small have a conjugate contained in a group in $\mathcal{H}$, so are universally elliptic. We shall associate a modular $\operatorname{group} \operatorname{Mod}(T) \subset \operatorname{Out}(T) \subset \operatorname{Out}(G)$ to such a tree $T$.

Lemma 5.1. If $v$ is a flexible $Q H$ vertex with finite fiber, then any elementary subgroup of $G_{v}$ is virtually cyclic.

Recall (Definition 2.7) that $G_{v}$ maps onto $\pi_{1}(\Sigma)$ with finite kernel $F$; flexibility of $G_{v}$ is equivalent to the 2-orbifold $\Sigma$ containing an essential 1-suborbifold. Since $T$ is only assumed to be relative to $\mathcal{H}$, Definition 2.7 only restricts intersections of $G_{v}$ with conjugates of groups in $\mathcal{H}$.

Proof. Assume on the contrary that some subgroup $E<G_{v}$ is elementary, but not virtually cyclic. Then $E$ contains $F_{2}$ and is parabolic. As pointed out before, our assumption on $\mathcal{H}$ implies that $E$ is universally elliptic. This contradicts Remark 2.9 saying that such a group has to be virtually cyclic. 
Remark. If $G_{v}$ is a flexible $\mathrm{QH}$ vertex stabilizer with elementary fiber $F$, then $F$ is finite. Indeed, if $F$ is infinite, then $G_{v}$ is elementary by almost malnormality of maximal elementary subgroups (Corollary 3.2). Since it contains $F_{2}$, it is universally elliptic, contradicting flexibility.

Definition 5.2. We say that a vertex $v$ of $T$ (or of $\Gamma=T / G$ ) is modular if $G_{v}$ is flexible and $\mathrm{QH}$ (relative to $\mathcal{H}$ ) with finite fiber, or $G_{v}$ is elementary. Note that $G_{v}$ cannot be both.

Recall (Subsection 2.6) the maps $\rho_{v}: \operatorname{Out}^{0}(T) \rightarrow \operatorname{Out}\left(G_{v}\right)$ defined on the finite index subgroup of $\operatorname{Out}(T)$ consisting of automorphisms acting trivially on $\Gamma=T / G$.

Definition 5.3. We define $\operatorname{Mod}(T)$ by saying that $\Phi \in \operatorname{Out}^{0}(T)$ belongs to $\operatorname{Mod}(T)$ if it satisfies the following conditions:

- if $v$ is not modular, $\rho_{v}(\Phi)$ is trivial;

- if $G_{v}$ is elementary, $\rho_{v}(\Phi) \in \operatorname{Out}\left(G_{v} ; \operatorname{Inc}_{v}^{(\mathrm{t})}\right)$; in other words, $\rho_{v}(\Phi)$ acts on each incident edge group as a conjugation;

- if $G_{v}$ is QH with finite fiber, and flexible, then $\rho_{v}(\Phi) \in \operatorname{Out}\left(G_{v} ; \mathcal{B}_{v}^{(\mathrm{t})}\right)$, with $\mathcal{B}_{v}$ consisting of full preimages of boundary subgroups of $\pi_{1}(\Sigma)$ as in Definition 2.8.

Note that $\operatorname{Mod}(T)$ contains the group of twists $\mathcal{T}(T)$, and that automorphisms in $\operatorname{Mod}(T)$ need not preserve $\mathcal{H}$.

We have assumed that $G$ is one-ended relative to $\mathcal{H}$, so we can consider the canonical elementary JSJ tree $T_{\text {can }}$ relative to $\mathcal{P} \cup \mathcal{H}$ as in Subsection 3.3. Note that $\operatorname{Mod}\left(T_{\text {can }}\right)$ has finite index in $\operatorname{Out}(G ; \mathcal{P})$ when $\mathcal{H}=\mathcal{P}$ (it contains the group $\operatorname{Out}^{1}(G ; \mathcal{P})$ defined in Subsection 4.2, possibly strictly because of vertices with $G_{v}$ virtually cyclic).

Theorem 5.4. Let $G$ be hyperbolic relative to $\mathcal{P}=\left\{P_{1}, \ldots, P_{n}\right\}$, with each $P_{i}$ finitely generated, not virtually cyclic. Let $\mathcal{H}$ be a family of subgroups such that every $P_{i}$ which contains $F_{2}$ is contained in a group of $\mathcal{H}$, and $G$ is one-ended relative to $\mathcal{H}$.

If $T$ is any elementary splitting of $G$ relative to $\mathcal{H}$, then $\operatorname{Mod}(T) \subset \operatorname{Mod}\left(T_{\mathrm{can}}\right)$, where $T_{\text {can }}$ is the canonical elementary JSJ tree relative to $\mathcal{P} \cup \mathcal{H}$.

This applies in particular if $G$ is one-ended and no $P_{i}$ contains $F_{2}$ (taking $\mathcal{H}=\emptyset$ ), or if $G$ is one-ended relative to an arbitrary $\mathcal{P}$ and we restrict to splittings relative to $\mathcal{P}$ (taking $\mathcal{H}=\mathcal{P}$ ). The theorem will be proved in the next subsection. 
Remark 5.5. Rather than defining $\operatorname{Mod}(T)$ by imposing conditions on the action on vertex groups, as we just did, one could define it by giving generators: twists around edges, and certain automorphisms of vertex groups. This would yield a slightly smaller group $\operatorname{Mod}^{\prime}(T)$ : its intersection with $\operatorname{ker} \rho$ is $\mathcal{T}$, whereas $\operatorname{Mod}(T)$ contains all of $\operatorname{ker} \rho$. Theorem 5.4 (and Theorem 5.6 below) also hold with this more restrictive definition, since $\operatorname{Mod}^{\prime}\left(T_{\text {can }}\right)=\operatorname{Mod}\left(T_{\text {can }}\right)$ by Assertion 2 of Lemma 2.12.

There is a similar statement for torsion-free CSA groups (recall that $G$ is CSA if centralizers of non-trivial elements are abelian and malnormal). We now consider abelian splittings of $G$. A vertex $v$ is modular if $G_{v}$ is either abelian or $\mathrm{QH}$ as above (in this case $F$ is trivial and $\Sigma$ is a surface). The definition of $\operatorname{Mod}(T)$ is the same (with elementary replaced by abelian). The tree $T_{\text {can }}$ is the canonical abelian JSJ tree relative to non-cyclic abelian subgroups; it is also the tree of cylinders of the (non-relative) abelian JSJ deformation space (see Theorem 11.1 of [36]).

Theorem 5.6. Let $G$ be a finitely generated, torsion-free, one-ended, CSA group. If $T$ is any splitting of $G$ over abelian groups, then $\operatorname{Mod}(T) \subset \operatorname{Mod}\left(T_{\text {can }}\right)$, where $T_{\mathrm{can}}$ is the tree of cylinders of the abelian JSJ deformation space.

Example 5.7. Let $G$ be the Baumslag-Solitar group

$$
\mathrm{BS}(2,4)=\left\langle a, b \mid b a^{2} b^{-1}=a^{4}\right\rangle .
$$

Any splitting of $G$ as a graph of infinite cyclic groups is a cyclic JSJ decomposition of $G[25,35]$. Its modular group coincides with its group of twists, and is a finite abelian group (see [47]). But the JSJ deformation space of BS $(2,4)$ is quite large [11], and JSJ splittings of BS $(2,4)$ may have modular groups of arbitrarily large order. In particular, there is no splitting whose modular group contains all others.

Example 5.8. Even if $G$ is as in Theorem 5.4, one cannot replace $T_{\text {can }}$ by an arbitrary tree in its deformation space: there exists such trees whose modular group is not maximal (even up to finite index).

Indeed, let $G=A_{1} *_{C_{1}} B *_{C_{2}} A_{2}$, where:

- $A_{1}$ and $A_{2}$ are torsion-free hyperbolic groups with no cyclic splitting;

- $C_{i}$ is a maximal infinite cyclic subgroup of $A_{i}$;

- $B$ is torsion-free, hyperbolic relative to a subgroup

$$
\widehat{C}=C_{1} \oplus C_{2} \oplus \mathbb{Z} \simeq \mathbb{Z}^{3},
$$

and does not split over an abelian group.

The group $G$ is hyperbolic relative to $\widehat{C}$ by [15]. 
The graph of groups

$$
A_{1} \frac{\stackrel{B}{\mid} C_{1} C_{1} \oplus C_{2}}{\oplus C_{2} \frac{}{C_{2}} A_{2}}
$$

is an elementary JSJ decomposition of $G$ (both absolute and relative to $\widehat{C}$ ) because its vertex groups are universally elliptic (see Lemma 4.7 of [35]). Given any $z \in \hat{C} \backslash\left(C_{1} \oplus C_{2}\right)$, the automorphism $\tau$ defined as the identity on $A_{1}$ and $B$ and as conjugation by $z$ on $A_{2}$ is not an automorphism of this graph of groups. But $\tau$ is a twist of $T_{\text {can }}$, which is the Bass-Serre tree of the graph of groups below.

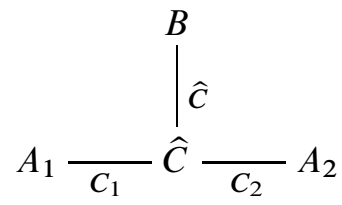

5.2. Proof of Theorem 5.4. We prove Theorem 5.4. The proof of Theorem 5.6 is similar and left to the reader. The main difference in the context of a general CSA group is that we have no version of Theorem 3.9 saying that a rigid vertex group only has finitely many outer automorphisms. But the proof given below does not use Theorem 3.9.

By Theorem 13.1 of [36], the trees $T_{\text {can }}$ and $T$ are compatible: they have a common refinement $\hat{T}$ (as defined in Subsection 2.3). We may assume that no edge of $\hat{T}$ is collapsed in both $T_{\text {can }}$ and $T$ (so $\hat{T}$ is the least common multiple of $T_{\text {can }}$ and $T$ as defined in Section 3 of [36]). The tree $\widehat{T}$ has elementary edge stabilizers and is relative to $\mathcal{H}$ since $T_{\text {can }}$ and $T$ are (Proposition 3.22 of [36]).

We first claim that $T_{\text {can }}$ is $\operatorname{Mod}(T)$-invariant. To see this, it suffices to show that the image of an infinite group $J \in \mathcal{P} \cup \mathcal{H}$ by a modular automorphism $\Phi$ has a finite index subgroup which is contained (up to conjugacy) in a group belonging to $\mathcal{P} \cup \mathcal{H}$.

If $J$ is a small $P_{i}$, its image is elementary and not virtually cyclic, so is parabolic. If $J$ is a $P_{i}$ containing $F_{2}$, it is contained in a group of $\mathcal{H}$, so we only have to consider groups $J \in \mathcal{H}$. Such a group fixes a vertex $v$ in $T$, and $G_{v}$ is $\Phi$-invariant. We distinguish three cases.

If $v$ is non-modular, $\Phi$ acts trivially on $J$. If $v$ is a flexible QH vertex, then $J$ is contained in a group of $\mathcal{B}_{v}$, hence $\Phi$-invariant. Now suppose that $J$ is contained in an elementary $G_{v}$. If $G_{v}$ contains $F_{2}$, it is a $\Phi$-invariant group contained in a group of $\mathcal{H}$, so the image of $J$ is contained in a group of $\mathcal{H}$. The case when $G_{v}$ is 
small but not virtually cyclic has been dealt with before. If $G_{v}$ is virtually cyclic, $\Phi(J)$ has a finite index subgroup contained in $J$. This completes the proof of the claim.

Let $\Phi \in \operatorname{Mod}(T)$. The heart of the proof of the theorem is to study the action of $\Phi$ on a non-elementary vertex stabilizer $G_{v}$ of $T_{\text {can }}$ (it is rigid or QH). In particular, given an edge $e=v w$ in $T_{\text {can }}$, we show that $\Phi$ has a representative $\alpha$ leaving $G_{v}$ invariant and equal to the identity on $G_{e}$.

We have defined $T_{\text {can }}$ as a JSJ tree relative to $\mathcal{P} \cup \mathcal{H}$. When $T_{\text {can }}$ is viewed as relative to $\mathcal{H}$ only, a flexible $\mathrm{QH}$ vertex remains flexible $\mathrm{QH}$. It follows from Sections 8 and 13 of [36] that a rigid (non-elementary) vertex stabilizer $G_{v}$ of $T_{\text {can }}$ remains universally elliptic relative to $\mathcal{H}$. The argument goes as follows: if $T$ is an elementary splitting relative to $\mathcal{H}$, then $G_{v}$ is elliptic in its tree of cylinders $T_{c}$ because $T_{c}$ is relative to $\mathcal{H} \cup \mathcal{P}$ and $G_{v}$ is rigid relative to $\mathcal{H} \cup \mathcal{P}$; since groups elliptic in $T_{c}$ but not in $T$ are elementary (see Subsection 2.7), and $G_{v}$ is not, this implies that $G_{v}$ is elliptic in $T$.

We distinguish two cases.

Case 1: $v$ is rigid. Then $G_{v}$ is universally elliptic, so fixes a point $u$ in $T$, which is unique because edge stabilizers are elementary and $G_{v}$ is not. The group $G_{u}$ cannot be elementary. By Remark 2.9, it cannot be flexible $\mathrm{QH}$ because its subgroup $G_{v}$ is universally elliptic and non-elementary. Thus $u$ is not modular and $\Phi$ has a representative $\alpha$ equal to the identity on $G_{u}$, hence on $G_{v}$.

Case 2: $v$ is a flexible $\mathrm{QH}$ vertex of $T_{\text {can }}$. Let $e$ be an adjacent edge and $\hat{e}$ its lift to $\widehat{T}$. Recall that $G_{e}=G_{\hat{e}}$ is a maximal elementary subgroup of $G_{v}$. We define a point $\hat{v} \in \widehat{T}$ as follows. If $G_{v}$ is elliptic in $\widehat{T}$, we call $\hat{v}$ its unique fixed point. If it is not elliptic, its action on its minimal subtree in $\hat{T}$ is dual to a family of 1-suborbifolds of $\Sigma$ (see Lemma 7.4 of [35]). We let $\hat{v}$ be the point of that subtree closest to $\hat{e}$ (possibly an endpoint of $\hat{e}$ ).

The stabilizer of $\hat{v}$ is QH, associated to a suborbifold $\hat{\Sigma}$ of $\Sigma$ (if $\hat{\Sigma}$ contains no essential 1-suborbifold, $\hat{v}$ is a rigid vertex of $\hat{T}$ ). Note that $G_{\hat{v}}$ is non-elementary by Lemma 5.1. The stabilizer of $\hat{e}$, and also of edges between $\hat{e}$ and $\hat{v}$ if any, is contained in $G_{\hat{v}}$, in fact in the preimage of a boundary subgroup of $\pi_{1}(\Sigma)$ and $\pi_{1}(\hat{\Sigma})$.

Let $u$ be the image of $\hat{v}$ in $T$.

Subcase 2a: $u$ is not modular. Then $\Phi$ has a representative $\alpha$ equal to the identity on $G_{u}$, hence on $G_{\hat{v}}$ and on $G_{e}=G_{\hat{e}}$. Note that $\alpha$ leaves $G_{v}$ invariant because $\alpha$ is an automorphism of $T_{\text {can }}$ and $v$ is the only vertex of $T_{\text {can }}$ fixed by $G_{\hat{v}}$.

Subcase 2b: $G_{u}$ is QH with finite fiber. Then $G_{u}$ is elliptic in $T_{\text {can }}$ (see Proposition 7.13 of [35], which is valid in a relative setting), hence in $\widehat{T}$, cf. [36, Proposi- 
tion 3.22], so $G_{u}=G_{\hat{v}}$. Unfortunately, this argument says nothing about incident edge groups at $u$.

First suppose that $\hat{v}$ is an endpoint of $\hat{e}$. Choose an edge path with origin $\hat{v}$, starting with $\hat{e}$, such that all edges except the last one get collapsed to $u$ in $T$ (this path consists of the single edge $\hat{e}$ if $\hat{e}$ is not collapsed to a point in $T$ ). Call this last edge $\hat{e}^{\prime}$, and its initial vertex $a$. We have $G_{\hat{e}^{\prime}} \subset G_{a} \subset G_{u}=G_{\hat{v}}$, so $G_{\hat{e}^{\prime}} \subset G_{\hat{e}}$.

The group $G_{u}$ is QH with finite fiber, and $G_{\hat{e}^{\prime}}$ is an incident edge group. It is infinite by one-endedness, so is contained in a unique maximal elementary subgroup $C$ of $G_{u}$ (the preimage of a boundary subgroup of the underlying 2orbifold). Since $G_{\hat{e}^{\prime}} \subset G_{\hat{e}}$, we have $G_{\hat{e}} \subset C$. By definition of $\operatorname{Mod}(T)$, there is a representative $\alpha$ of $\Phi$ leaving $G_{u}$ invariant and equal to the identity on $C$, hence on $G_{e}=G_{\hat{e}}$. As above, $\alpha$ leaves $G_{v}$ invariant.

If there are edges between $\hat{v}$ and $\hat{e}$, call $\hat{e}^{\prime}$ the edge that contains $\hat{v}$. It is not collapsed to a point in $T$, since it is collapsed in $T_{\text {can }}$, so $G_{\hat{e}^{\prime}}$ is an incident edge group of $G_{u}$. We now have $G_{\hat{e}} \subset G_{\hat{e}^{\prime}} \subset C$ and we argue as in the previous case. This completes the analysis of the action of $\Phi$ on $G_{e}$ in case 2 .

Still in case 2 (i.e. assuming that $v$ is a flexible $\mathrm{QH}$ vertex stabilizer of $T_{\text {can }}$ ), we also need to understand the action of $\Phi$ on an element $B \in \mathcal{B}_{v}$ which is not an incident edge stabilizer. Such a $B$ contains a conjugate of an $H_{j}$ with finite index.

By minimality of $\widehat{T}$, the group $B$ fixes a $\mathrm{QH}$ vertex $\hat{v} \in \widehat{T}$. We then argue as above. In subcase $2 \mathrm{~b}$, we have $B \in \mathcal{B}_{u}$ (up to conjugacy) because $B$ contains a conjugate of $H_{j}$, so we can find $\alpha$ leaving $G_{u}$ invariant and equal to the identity on $B$ since $\Phi \in \operatorname{Mod}(T)$. This finishes case 2 .

We can now conclude. Consider $\Gamma_{\text {can }}=T_{\text {can }} / G$, and recall that $T_{\text {can }}$ is a tree of cylinders, so $\Gamma_{\text {can }}$ is bipartite, with edges joining a vertex $x \in V_{0}\left(\Gamma_{\text {can }}\right)$ carrying a non-elementary group to a vertex $Y \in V_{1}\left(\Gamma_{\text {can }}\right)$ carrying an elementary group. We know that $\Phi$ fixes each vertex $x \in V_{0}\left(\Gamma_{\text {can }}\right)$, and its action on $G_{x}$ is trivial if $x$ is not modular, in $\operatorname{Out}\left(G_{x} ; \mathcal{B}_{x}^{(\mathrm{t})}\right)$ if $G_{x}$ is $\mathrm{QH}$.

Since $T_{\text {can }}$ is a tree of cylinders, distinct edges of $\Gamma_{\text {can }}$ with origin $x$ in $V_{0}\left(\Gamma_{\text {can }}\right)$ carry groups which are not conjugate in $G_{x}$. As $\rho_{x}(\Phi) \in \operatorname{Out}\left(G_{x} ; \operatorname{Inc}_{x}^{(\mathrm{t})}\right)$, we deduce that $\Phi$ acts as the identity on edges of $\Gamma_{\text {can }}$ with origin $x$, hence on the whole of $\Gamma_{\text {can }}$. Thus $\Phi \in \operatorname{Out}^{0}\left(T_{\text {can }}\right)$.

There remains to check that $\rho_{Y}(\Phi) \in \operatorname{Out}\left(G_{Y} ; \operatorname{Inc}_{Y}^{(\mathrm{t})}\right)$ for $Y \in V_{1}\left(\Gamma_{\text {can }}\right)$. If $\varepsilon=(x, Y)$ is an adjacent edge, we have seen that $\Phi$ has a representative $\alpha$ equal to the identity on $G_{\varepsilon}$. Since $G_{\varepsilon}$ is infinite, $G_{Y}$ is the unique maximal elementary subgroup containing it, so $\alpha$ leaves $G_{Y}$ invariant. This completes the proof. 


\section{Induced automorphisms}

In this section $G$ is hyperbolic relative to $\mathcal{P}=\left\{P_{1}, \ldots, P_{n}\right\}$, and we study automorphisms of $P_{1}$ which are induced by automorphisms of $G$. We then apply this to the case when $G$ is hyperbolic and $H$ is a malnormal quasiconvex subgroup, viewing $G$ as hyperbolic relative to $H$.

Definition 6.1. Given families of subgroups $\mathcal{P}$ and $\mathcal{H}$, and a subgroup $Q$, we say that $\alpha \in \operatorname{Aut}(Q)$ is extendable to $G$ relative to $\mathcal{P}$ and $\mathcal{H}^{(\mathrm{t})}$ if it is the restriction to $Q$ of an automorphism of $G$ representing an element of $\operatorname{Out}\left(G ; \mathcal{P}, \mathcal{H}^{(\mathrm{t})}\right)$.

Being extendable only depends on the image of $\alpha$ in $\operatorname{Out}(Q)$, so we define the group of extendable automorphisms

$$
\operatorname{Out}\left(Q \uparrow\left(G ; \mathcal{P}, \mathcal{H}^{(\mathrm{t})}\right)\right) \subset \operatorname{Out}(Q) .
$$

We write $\operatorname{Out}(Q \uparrow(G ; \mathcal{P}))$ when $\mathcal{H}=\emptyset$, and $\operatorname{Out}(Q \uparrow G)$ for $\operatorname{Out}(Q \uparrow(G ; \emptyset))=$ $\operatorname{Out}(Q \uparrow(G ;\{Q\}))$.

If $Q$ equals its normalizer (for instance if $Q$ is an infinite maximal parabolic subgroup), there is a map $\operatorname{Out}(G ; Q) \rightarrow \operatorname{Out}(Q)$, and $\operatorname{Out}(Q \uparrow G)$ is its image.

Suppose that $P_{1}=G_{v}$ is a vertex group of a splitting of $G$ relative to $\mathcal{P}=$ $\left\{P_{1}, \ldots, P_{n}\right\}$, and $P_{1}$ contains no conjugate of $P_{i}$ for $i>1$ (this is automatic if no $P_{i}$ is finite). Then $\operatorname{Out}\left(P_{1} \uparrow(G ; \mathcal{P})\right)$ contains $\operatorname{Out}\left(G_{v} ; \operatorname{Inc}_{v}^{(\mathrm{t})}\right)$ (see Lemma 2.10). The following theorem says that virtually all extendable automorphisms occur in this fashion.

Theorem 6.2. Let $G$ be hyperbolic relative to $\mathcal{P}=\left\{P_{1}, \ldots, P_{n}\right\}$, with $P_{i}$ finitely generated and infinite, and $P_{i} \neq G$. Let $\mathcal{H}$ be a finite family of finitely generated subgroups of $G$. If $\operatorname{Out}\left(P_{1} \uparrow\left(G ; \mathcal{P}, \mathcal{H}^{(\mathrm{t})}\right)\right)$ is infinite, then:

(1) $P_{1}$ is a vertex group $G_{v}$ in an elementary JSJ decomposition $\Gamma$ relative to $\mathcal{P} \cup \mathcal{H}$. Edge groups of $\Gamma$ are finitely generated;

(2) The group $\operatorname{Out}\left(P_{1} \uparrow\left(G ; \mathcal{P}, \mathcal{H}^{(\mathrm{t})}\right)\right) \subset \operatorname{Out}\left(P_{1}\right)$ has a finite index subgroup equal to $\operatorname{Out}\left(P_{1} ; \mathcal{K}^{(\mathrm{t})}\right)$, where $\mathcal{K}=\operatorname{Inc}_{v} \cup \mathcal{H}_{\mid G_{v}}$ is a finite family of finitely generated subgroups of $P_{1}$ (the family of incident edge groups $\operatorname{Inc}_{v}$, and $\mathcal{H}_{\mid G_{v}}$, are defined in Subsection 2.4).

Since we do not assume that $G$ is one-ended relative to $\mathcal{P} \cup \mathcal{H}$, there is no canonical JSJ decomposition. 
Proof. • First assume that $G$ is one-ended relative to $\mathcal{P} \cup \mathcal{H}$. Consider the canonical elementary JSJ tree $T_{\text {can }}$ relative to $\mathcal{P} \cup \mathcal{H}$ as in Subsection 3.3.

Let $G_{v}$ be a vertex stabilizer containing $P_{1}$. It cannot be flexible $\mathrm{QH}$ because $P_{1}$ is not virtually cyclic (see Remark 2.9). If it is rigid (non-elementary), we have seen in Subsection 4.3 that the image of $\operatorname{Out}^{0}\left(G ; \mathcal{P}, \mathcal{H}^{(\mathrm{t})}\right)$ in $\operatorname{Out}\left(G_{v}\right)$ is finite (recall that $\operatorname{Out}^{0}\left(G ; \mathcal{P}, \mathcal{H}^{(\mathrm{t})}\right)$ is the finite index subgroup of $\operatorname{Out}\left(G ; \mathcal{P}, \mathcal{H}^{(\mathrm{t})}\right)$ acting trivially on $\left.T_{\text {can }} / G\right)$. Since $P_{1}$ equals its normalizer, this implies that $\operatorname{Out}\left(P_{1} \subsetneq\left(G ; \mathcal{P}, \mathcal{H}^{(\mathrm{t})}\right)\right)$ is finite, a contradiction. Thus $G_{v}$ is elementary, so we deduce $G_{v}=P_{1}$. This proves Assertion 1 in the one-ended case (edge stabilizers of $T_{\text {can }}$ are finitely generated by Lemma 3.7). Assertion 2 is also clear since $\left.\operatorname{Out}\left(P_{1}\right\rceil\left(G ; \mathcal{P}, \mathcal{H}^{(\mathrm{t})}\right)\right)$ is virtually $\operatorname{Out}\left(P_{1} ; \operatorname{Inc}_{v}^{(\mathrm{t})}, \mathcal{H}_{\mid G_{v}}^{(\mathrm{t})}\right)$ by Proposition 4.7.

- We now consider the general case, first assuming that $G$ is torsion-free. Let $F=G_{u}$ be the vertex stabilizer containing $P_{1}$ in a Grushko decomposition $S$ relative to $\mathcal{P} \cup \mathcal{H}$ (see Subsection 2.5), and let $\mathcal{P}_{\mid F}, \mathcal{H}_{\mid F}$ be the induced structures (see Definition 2.3); if $\mathcal{P} \cup \mathcal{H}=\left\{P_{1}\right\}$, then $F$ is simply the smallest free factor containing $P_{1}$.

Since $F$ is hyperbolic relative to $\mathcal{P}_{\mid F}$ by Lemma 3.5, and

$$
\operatorname{Out}\left(P_{1} \uparrow\left(G ; \mathcal{P}, \mathcal{H}^{(\mathrm{t})}\right)\right)=\operatorname{Out}\left(P_{1} \uparrow\left(F ; \mathcal{P}_{\mid F}, \mathcal{H}_{\mid F}^{(\mathrm{t})}\right)\right)
$$

because $F$ is a free factor (or by Remark 2.11), the results of the previous case apply. The group $P_{1}$ is a vertex group $G_{v}$ of a splitting $\Gamma_{F}$ of $F$, which may be used to refine $S$ to an elementary JSJ decomposition $\Gamma$ of $G$ having $G_{v}$ as a vertex group (see Subsection 8.1 of [35]). The families $\operatorname{Inc}_{v}$ and $\mathcal{H}_{\mid G_{v}}$ are the same for $\Gamma_{F}$ and $\Gamma$.

- If $G$ has torsion, we define $F=G_{u}$ and $\mathcal{P}_{\mid F}, \mathcal{H}_{\mid F}$ as above, using a StallingsDunwoody tree $S$ relative to $\mathcal{P} \cup \mathcal{H}$. The proof is technically more complicated because we cannot neglect the incident edge groups $\operatorname{Inc}_{u}$.

All Stallings-Dunwoody trees $S$ have a unique vertex stabilizer $G_{u(S)}$ equal to $F$, but the incident edge groups may vary. This was studied in Section 4 of [32], where we defined a "peripheral structure" for $F$. To state the relevant result, we choose a Stallings-Dunwoody tree $S$ for which the valence of $u(S)$ in the quotient graph of groups $S / G$ is minimal. Since no edge stabilizer is properly contained in a conjugate, it follows from Proposition 4.9 of [32] that the incident structure $\operatorname{Inc}_{u(S)}$ does not depend on the choice of such an $S$ (in trees with non-minimal valence, there may be more incident edge groups; such a group is contained in a group belonging to $\left.\operatorname{Inc}_{u(S)}\right)$. We fix $S$, and from now on we write $u$ rather than $u(S)$, so $F=G_{u}$. 
Any automorphism representing an element of $\operatorname{Out}\left(G ; \mathcal{P}, \mathcal{H}^{(\mathrm{t})}\right)$ and leaving $P_{1}$ invariant also leaves $F$ invariant. Since $P_{1}$ and $F$ are equal to their normalizers, $\left.\operatorname{Out}\left(P_{1}\right\rceil\left(G ; \mathcal{P}, \mathcal{H}^{(\mathrm{t})}\right)\right)$ is the image of the map

$$
p: \operatorname{Out}\left(G ; \mathcal{P}, \mathcal{H}^{(\mathrm{t})}\right) \longrightarrow \operatorname{Out}\left(P_{1}\right),
$$

and $p$ factors through

$$
\rho_{u}: \operatorname{Out}\left(G ; \mathcal{P}, \mathcal{H}^{(\mathrm{t})}\right) \longrightarrow \operatorname{Out}(F) .
$$

By Remark 2.11, the image of $\rho_{u}$ contains $\operatorname{Out}\left(F ; \operatorname{Inc}_{u}^{(\mathrm{t})}, \mathcal{P}_{\mid F}, \mathcal{H}_{\mid F}^{(\mathrm{t})}\right)$ and is contained in $\operatorname{Out}\left(F ; \mathcal{P}_{\mid F}, \mathcal{H}_{\mid F}^{(\mathrm{t})}\right)$.

Our choice of $S$ implies that automorphisms in the image of $\rho_{u}$ preserve $\operatorname{Inc}_{u}$ globally. Since $\mathrm{Inc}_{u}$ consists of finitely many finite subgroups of $F$ (well defined up to conjugacy), the index of $\operatorname{Out}\left(F ; \operatorname{Inc}_{u}^{(\mathrm{t})}, \mathcal{P}_{\mid F}, \mathcal{H}_{\mid F}^{(\mathrm{t})}\right)$ in $\operatorname{Out}\left(F ; \mathcal{P}_{\mid F}, \mathcal{H}_{\mid F}^{(\mathrm{t})}\right)$ is finite. It therefore suffices to study the image of

$$
q: \operatorname{Out}\left(F ; \operatorname{Inc}_{u}^{(\mathrm{t})}, \mathcal{P}_{\mid F}, \mathcal{H}_{\mid F}^{(\mathrm{t})}\right) \longrightarrow \operatorname{Out}\left(P_{1}\right),
$$

and to show that it is virtually $\operatorname{Out}\left(P_{1} ; \mathcal{K}^{(\mathrm{t})}\right)$.

The group $F=G_{u}$ is hyperbolic relative to the family $\mathcal{P}_{\mid F}$ (see Lemma 3.5), and one-ended relative to $\mathcal{P}_{\mid F} \cup \mathcal{H}_{\mid F}$. Since the image of $q$ is infinite, we have seen that $P_{1}$ is a vertex group $G_{v}$ in the canonical elementary JSJ decomposition $\Gamma_{\text {can }}$ of $F$ relative to $\mathcal{P}_{\mid F} \cup \mathcal{H}_{\mid F}$. One obtains an elementary JSJ tree $T$ of $G$ relative to $\mathcal{P} \cup \mathcal{H}$ by refining $S$ using JSJ decompositions of vertex groups (see Subsection 8.1 of [35]), so Assertion 1 is proved.

Moreover, $\operatorname{Out}\left(P_{1} \subsetneq\left(G ; \mathcal{P}, \mathcal{H}^{(\mathrm{t})}\right)\right)$ is virtually $\operatorname{Out}\left(P_{1} ; \mathcal{K}^{\prime(\mathrm{t})}\right)$, where $\mathcal{K}^{\prime}$ is the union of $\operatorname{Inc}_{v}$ (the incident edge groups of $P_{1}$ in $T_{\text {can }}$ ) and $\left(\operatorname{Inc}_{u} \cup \mathcal{H}_{\mid F}\right)_{\mid P_{1}}$. We now show that $\mathcal{K}^{\prime}=\mathcal{K}$ if we construct $T$ carefully.

When $S$ is refined to yield $T$, the vertex $u$ is replaced by $T_{\text {can }}$. There is some freedom in the way edges of $S$ containing $u$ are attached to $T_{\text {can }}$ : an edge $e$ may be attached to any vertex of $T_{\text {can }}$ which is fixed by $G_{e}$. We may therefore assume that, if $e$ is an edge of $T \backslash T_{\text {can }}$ attached to $v$, then $v$ is the only fixed point of $G_{e}$ in $T_{\text {can }}$.

The family $\left(\mathcal{H}_{\mid F}\right)_{\mid P_{1}}$ is defined viewing $F$ as a vertex group of $S$, and then $P_{1}$ as a vertex group of $T_{\text {can }}$. Since groups in $\mathcal{H}$ are infinite and edge stabilizers of $S$ are finite, $\left(\mathcal{H}_{\mid F}\right)_{\mid P_{1}}$ equals $\mathcal{H}_{\mid P_{1}}$, defined viewing $P_{1}$ as a vertex group of $T$. We complete the proof by showing that $\operatorname{Inc}_{v} \cup\left(\operatorname{Inc}_{u}\right)_{\mid P_{1}}$ is the family of incident edge groups in $P_{1}=G_{v}$ viewed as a vertex stabilizer of $T$. 
There are two types of incident edge groups of $G_{v}$ in $T$. Those fixing edges in $T_{\text {can }}$ are precisely those in $\operatorname{Inc}_{v}$. Because of the way we constructed $T$, those fixing edges in $T \backslash T_{\text {can }}$ have $v$ as unique fixed point in $T_{\text {can }}$, they are the groups in $\left(\operatorname{Inc}_{u}\right)_{\mid P_{1}}$ (see Definition 2.3).

If $G$ is (absolutely) hyperbolic, and $P$ is a subgroup, then $G$ is hyperbolic relative to $\{P\}$ if (and only if) $P$ is quasiconvex and almost malnormal, see [6, Theorem 7.11] or [52]. If so, Theorem 6.2 applies and describes $\operatorname{Out}(P \uparrow G)$, the automorphisms of $P$ which extend to $G$.

Corollary 6.3. Let $P$ be a quasiconvex, almost malnormal subgroup of a hyperbolic group $G$, with $P \neq G$.

- If $\operatorname{Out}(P \uparrow G)$ is infinite, then $P$ is a vertex group in a splitting of $G$ with finitely generated edge groups, and $\operatorname{Out}(P \uparrow G)$ is virtually $\operatorname{Out}\left(P ; \mathcal{K}^{(\mathrm{t})}\right)$ with $\mathcal{K}$ the family of incident edge groups (a finite family of finitely generated subgroups of $P$ ).

- If $P$ is torsion-free, then $\operatorname{Out}(P \uparrow G)$ has a finite index subgroup with a finite classifying space.

Proof. The first assertion follows from Theorem 6.2. Being quasiconvex, $P$ is a hyperbolic group. It is proved in [37] that, if $P$ is a torsion-free hyperbolic group and $\mathcal{K}$ is an arbitrary family of subgroups, then $\operatorname{Out}\left(P ; \mathcal{K}^{(\mathrm{t})}\right)$ has a finite index subgroup with a finite classifying space.

If $G=F_{n}$, every finitely generated subgroup is quasiconvex (it is a virtual retract by [38]), so we get:

Corollary 6.4. If $P \subset F_{n}$ is finitely generated and malnormal, then $\operatorname{Out}\left(P \uparrow F_{n}\right)$ is virtually $\operatorname{Out}\left(P ; \mathcal{K}^{(\mathrm{t})}\right)$ for some finite family $\mathcal{K}$ of finitely generated subgroups of $P$. It has a finite index subgroup with a finite classifying space.

This is a partial answer to a question that was asked by D. Calegari. Note that the proof uses JSJ decompositions over groups which are not small.

Example 6.5. Let $P \subset F_{n}$ be a characteristic subgroup of finite index, with $n \geq 3$. Then $\operatorname{Out}(P \nmid G)$ is not virtually of the form $\operatorname{Out}\left(P ; \mathcal{K}^{(\mathrm{t})}\right)$ because there exist automorphisms of $F_{n}$ with no nontrivial periodic conjugacy class. There are similar examples with $P$ of infinite index. 


\section{Groups with infinitely many automorphisms}

In this section, we characterize those relatively hyperbolic groups whose automorphism group is infinite.

In the first subsection, we point out that determining whether $\operatorname{Out}(G)$ is infinite or not is relatively easy when $G$ is torsion-free or one-ended. In particular, we give a complete answer for toral relatively hyperbolic groups.

The most interesting case is thus when $G$ has torsion and splits over a finite group. For instance, virtually free groups with Out finite were determined by M. Pettet [56]. We will give a different characterization (see Example 7.7).

If $G$ is hyperbolic relative to $\mathcal{P}$, we will show in Subsection 7.2 that the group $\operatorname{Out}\left(G ; \mathcal{P}^{(\mathrm{t})}\right)$ of automorphisms which act trivially on each parabolic subgroup is infinite if and only if $G$ has an elementary splitting relative to $\mathcal{P}$ whose group of twists is infinite.

In Subsection 7.3, we get a characterization for the full group $\operatorname{Out}(G ; \mathcal{P})$ being infinite: this happens if and only if $G$ has an elementary splitting relative to $\mathcal{P}$ whose group of twists is infinite, or in which a maximal parabolic subgroup $P$ occurs as a vertex group and $P$ has infinitely many outer automorphisms acting trivially on incident edge groups (such automorphisms extend to $G$ ).

When $G$ is hyperbolic, we show in Subsection 7.4 that $\operatorname{Out}(G)$ being infinite is equivalent to $G$ having a splitting over a maximal virtually cyclic group with infinite center; this is decidable algorithmically.

\subsection{Torsion free groups. We first note:}

Lemma 7.1. If a torsion-free, finitely generated, group $G$ is a non-trivial free product, then $\operatorname{Out}(G)$ is infinite.

Proof. Write $G=A * B$. If $a \in A$ is not central, the automorphism of $G$ equal to conjugation by $a$ on $A$ and to the identity on $B$ has infinite order in $\operatorname{Out}(G)$. Assuming that $\operatorname{Out}(G)$ is finite, we deduce that $Z(A)$ has finite index in $A$, so $A$ is abelian because $[A, A]$ is finite by a result due to Schur [57, 10.1.4]. Similarly, $B$ is abelian. Moreover, $\operatorname{Out}(A)$ and $\operatorname{Out}(B)$ are finite, so $A=B=\mathbb{Z}$. This is a contradiction since $\operatorname{Out}(\mathbb{Z} * \mathbb{Z})$ is infinite.

Thus, for torsion-free groups, infiniteness of $\operatorname{Out}(G)$ is only interesting for oneended groups. One can get a similar result in a relative setting. 
Proposition 7.2. Let $G$ be a finitely generated, non-cyclic, torsion-free group, and $\mathcal{H}$ a finite collection of finitely generated subgroups. If the Grushko decomposition of $G$ relative to $\mathcal{H}$ is non-trivial, and not an amalgam $G=A_{1} * A_{2}$ with $A_{1}, A_{2}$ abelian, then $\operatorname{Out}\left(G ; \mathcal{H}^{(\mathrm{t})}\right)$ is infinite.

Remark 7.3. If the Grushko decomposition $\Gamma$ relative to $\mathcal{H}$ is $G=A_{1} * A_{2}$ with $A_{1}, A_{2}$ abelian, then $\operatorname{Out}\left(G ; \mathcal{H}^{(\mathrm{t})}\right)$ is finite if and only if, for $i=1,2$, the subgroup of $A_{i}$ generated by subgroups conjugate to a group in $\mathcal{H}$ has finite index. This is because $\Gamma$ is $\operatorname{Out}\left(G ; \mathcal{H}^{(\mathrm{t})}\right)$-invariant by [24] (its Bass-Serre tree is the unique reduced tree in its deformation space). Twists are trivial because $A_{1}$ and $A_{2}$ are abelian, so $\operatorname{Out}\left(G ; \mathcal{H}^{(\mathrm{t})}\right)$ is infinite if and only if $A_{1}$ or $A_{2}$ has infinitely many automorphisms acting trivially on $\mathcal{H}_{\mid A_{i}}$.

Proof. Assume that $\operatorname{Out}\left(G ; \mathcal{H}^{(\mathrm{t})}\right)$ is finite, and let $\Gamma$ be a reduced Grushko decomposition of $G$ relative to $\mathcal{H}$. We assume that $\Gamma$ is non-trivial and we show that it is an amalgam as in the proposition.

We first note that $G$ cannot split relative to $\mathcal{H}$ as an $\mathrm{HNN}$ extension $G=$ $A *_{\{1\}}$ over the trivial group. Indeed, the group of twists of this HNN extension is isomorphic to $(A \times A) / Z(A)$, with $Z(A)$ embedded diagonally, so contains the infinite group $A$, a contradiction. It follows that $\Gamma$ is a tree of groups.

The proof of Lemma 7.1 shows that, whenever $G$ splits as a free product $A * B$ relative to $\mathcal{H}$, then $A$ and $B$ are abelian: otherwise the group of twists of the splitting is infinite. Since $\Gamma$ is reduced, it follows that it is an amalgam $G=A_{1} * A_{2}$ with $A_{1}, A_{2}$ abelian: if $\Gamma$ has more than one edge, collapsing an edge provides a decomposition with a non-abelian vertex group.

Let now $G$ be hyperbolic relative to $\mathcal{P}=\left\{P_{1}, \ldots, P_{n}\right\}$, with $P_{i}$ finitely generated, not virtually cyclic.

If $G$ is one-ended relative to $\mathcal{P}$, one can read infiniteness of $\operatorname{Out}(G ; \mathcal{P})$ from the JSJ decomposition thanks to Theorem 4.3: $\operatorname{Out}(G ; \mathcal{P})$ is finite if and only if the canonical elementary JSJ decomposition relative to $\mathcal{P}$ has no flexible $\mathrm{QH}$ vertex with infinite mapping class group, the parabolic subgroups $P_{j}$ appearing as vertex stabilizers have $\operatorname{Out}\left(P_{j} ; \operatorname{Inc}_{P_{j}}^{(\mathrm{t})}\right)$ finite, and the group of twists is finite. We may be more specific under additional conditions on the parabolic subgroups.

Proposition 7.4. Let $G$ be a non-abelian toral relatively hyperbolic group. The following are equivalent.

(1) $\operatorname{Out}(G)$ is finite.

(2) Every non-trivial abelian one-edge splitting of $G$ is an amalgam $A *_{C} B$ with $C$ of finite index in $A$ or $B$. 
(3) G has no non-trivial splitting over an abelian subgroup stable under taking roots.

(4) $G$ is freely indecomposable and its canonical abelian JSJ decomposition $\Gamma_{\text {can }}$ relative to non-cyclic abelian subgroups satisfies the following:

- $\Gamma_{\text {can }}$ consists of a central vertex, possibly connected to terminal vertices carrying an abelian group;

- the central vertex is rigid, or QH with underlying surface $\Sigma$ homeomorphic to a pair of pants or a twice punctured projective plane;

- at each terminal vertex, the incident edge group has finite index in the vertex group.

A subgroup $A$ is stable under taking roots if $g \in A$ whenever $g^{n} \in A$ for some $n \geq 2$ (this is also called pure, or isolated).

The pair of pants and the twice punctured projective plane appear in this statement because they are the only compact hyperbolic surfaces with finite mapping class group (see the end of Subsection 4.1). The fundamental group of a pair of pants is rigid. The fundamental group of a twice punctured projective plane has two (incompatible) cyclic splittings relative to the boundary (it is flexible), but none over a maximal cyclic subgroup.

Automorphisms of toral relatively hyperbolic groups were considered in [16], and some of the equivalences in Proposition 7.4 follow from their results (note for instance that a splitting as in (3) is an essential splitting in the sense of their Definition 3.30).

Proof. If $G$ is a free product, (2) and (3) are false, and so is (1) by Lemma 7.1. We therefore assume that $G$ is freely indecomposable.

We prove (1) $\Rightarrow$ (2) by assuming that (2) does not hold, and showing that $\operatorname{Out}(G)$ is infinite. If $G$ is an HNN extension over an abelian group, or an amalgam with $A$ and $B$ non-abelian, the group of twists of the splitting is infinite. If $G=$ $A *_{C} B$ with $A$ abelian containing $C$ with infinite index, $\operatorname{Out}(G)$ is infinite because $A$ has nontrivial automorphisms equal to the identity on $C$.

It is clear that (2) implies (3). To prove that (3) implies (2), first assume that $G=A *_{C} B$ with $C$ abelian of infinite index in both $A$ and $B$. Let $\widehat{C}$ be the set of all roots of elements of $C$, an abelian subgroup containing $C$ with finite index (recall that all abelian subgroups of $G$ are cyclic or parabolic, hence finitely generated). Since $\widehat{C}$ is elliptic in the amalgam, up to exchanging the role of $A$ and $B$, we can assume that $\widehat{C}<A$. Then $G=A *_{\hat{C}}\langle B, \widehat{C}\rangle$ is a decomposition 
contradicting (3). The case of an HNN extension is similar, but we do not need the hypothesis that $C$ has infinite index in $A$.

If (4) does not hold, we construct a splitting contradicting (2). If $\Gamma_{\text {can }}$ has a flexible QH vertex, and if the underlying surface is not a twice punctured projective plane, then one simply considers the cyclic splitting dual to a 2-sided essential simple closed curve not bounding a Möbius band. The other possibility is that $\Gamma_{\text {can }}$ has a vertex $v$ carrying an abelian group such that either $v$ has valence $\geq 2$, or $v$ is terminal with the edge group of infinite index in $G_{v}$. One gets the required splitting by collapsing edges of $\Gamma_{\text {can }}$.

We have proved (1) $\Rightarrow(2) \Rightarrow$ (4) and (2) $\Leftrightarrow(3)$. We conclude by deducing from the exact sequence of Corollary 4.4 that $\operatorname{Out}(G)$ is finite if (4) holds. The groups $\mathrm{GL}_{r_{k}, n_{k}}(\mathbb{Z})$ are trivial because $r_{k}=0$ by the finiteness assumption at terminal vertices. The mapping class group of $\Sigma$ is finite. Twists are trivial because terminal vertices carry an abelian group.

Proposition 7.5. Let $G$ be torsion-free, hyperbolic relative to nilpotent subgroups. Assume that $G$ is not nilpotent. If $\operatorname{Out}(G)$ is finite, then $G$ is freely indecomposable and its canonical JSJ decomposition $\Gamma_{\text {can }}$ over nilpotent groups relative to noncyclic nilpotent subgroups consists of a central vertex which is rigid, or QH with underlying surface $\Sigma$ homeomorphic to a pair of pants or a twice punctured projective plane, possibly connected to terminal vertices carrying a nilpotent group.

Proof. We may assume that no $P_{i}$ is cyclic, so $\operatorname{Out}(G)=\operatorname{Out}(G ; \mathcal{P})$. As above, $G$ is freely indecomposable and there is no $\mathrm{QH}$ vertex other than those mentioned. Recall that an infinite torsion-free nilpotent group has infinite center. As in the proof of Corollary 4.4, the group of twists of $\Gamma_{\text {can }}$ contains the direct product $\prod\left(Z\left(G_{v}\right)\right)^{\left|E_{v}\right|-1}$ taken over vertices carrying a nilpotent group, so is infinite as soon as there is a vertex with valence $\left|E_{v}\right| \geq 2$.

\subsection{Infinity of marked automorphisms}

Theorem 7.6. Let $G$ be hyperbolic relative to $\mathcal{P}=\left\{P_{1}, \ldots, P_{n}\right\}$, with each $P_{i}$ finitely generated. Then $\operatorname{Out}\left(G ; \mathcal{P}^{(\mathrm{t})}\right)$ is infinite if and only if there is a splitting of $G$ over finitely generated elementary subgroups, relative to $\mathcal{P}$, with an infinite group of twists $\mathcal{T}$ (see Subsection 2.6).

More generally, if $\mathcal{Q}$ is a finite family of finitely generated subgroups with $\mathcal{P} \subset \mathcal{Q}$, the same characterization holds for $\operatorname{Out}\left(G ; Q^{(\mathrm{t})}\right)$, with a splitting relative to $Q$. 
By Lemma 2.15, the splittings may be assumed to have only one edge. The proof will be given at the end of the subsection.

Example 7.7. Consider the virtually free group $G=D_{4} *_{C} D_{6} *_{C} D_{4}$, where $D_{n}$ is the dihedral group of order $n$ and $C$ has order 2 (note that $C$ is central in $D_{4}$, but equal to its centralizer in $D_{6}$ ). For this two-edge splitting $\Gamma$, and any splitting obtained by collapsing an edge, the group of twists is finite. The one-edge splitting $\Delta$ given by the amalgam $G=D_{6} *_{C}\left(D_{4} *_{C} D_{4}\right)$, however, has a twist of infinite order. The Bass-Serre tree of $\Delta$ is the tree of cylinders of the Bass-Serre tree $T$ of $\Gamma$, and Assertion 1 of Proposition 7.10 holds in this case. Compare [56].

When $G$ is hyperbolic, the group of twists of the splitting provided by Theorem 7.6 contains an element of infinite order (see also Theorem 7.14). The following example shows that this does not hold for general relatively hyperbolic groups.

Example 7.8. Let $G=B_{1} * B_{2}$ be the free product of two infinite torsion groups with trivial center. It is hyperbolic relative to $\mathcal{P}=\left\{B_{1}, B_{2}\right\}$, and $\operatorname{Out}\left(G ; \mathcal{P}^{(\mathrm{t})}\right)$ is infinite. But no splitting over elementary subgroups has a twist of infinite order, as we now show. By way of contradiction, suppose that some $Z_{G_{o(e)}}\left(G_{e}\right)$ contains an element of infinite order. The group $G_{e}$ is trivial, or contains a torsion element $g \neq 1$, or is isomorphic to $\mathbb{Z}$. It cannot be trivial since $G_{o(e)}$ would then be a torsion group. The existence of a torsion element $g$ also leads to a contradiction since the centralizer of such a $g$ is a torsion group. We conclude by observing that $G$ does not split over $\mathbb{Z}$ : if it does, an equivariant map from the Bass-Serre tree of the amalgam $B_{1} * B_{2}$ to that of the splitting maps vertices to vertices and must be locally injective, hence globally injective, a contradiction.

Before proving Theorem 7.6, we note the following fact, which follows from the presentation of $\mathcal{T}$ recalled in Subsection 2.6.

Lemma 7.9. Let $G$ be a relatively hyperbolic group. Let $\Gamma$ be a one-edge splitting of $G$ over a virtually cyclic group $G_{e}$ with infinite center, with $G_{e}$ not parabolic. Any element of infinite order in $Z\left(G_{e}\right)$ defines a twist which has infinite order in $\operatorname{Out}(G)$, unless $\Gamma$ is an amalgam and one of the vertex groups is virtually cyclic with infinite center.

The following result is a key step in the proof of Theorem 7.6. 
Proposition 7.10. Let $T$ be a non-trivial tree with edge stabilizers of constant finite cardinality $k$. Let $T_{c}$ be its tree of cylinders for the equality equivalence relation (see Subsection 2.7). Then at least one of the following holds:

(1) $T_{c}$ is nontrivial and its edge stabilizers are finite;

(2) Thas a collapse $T^{\prime}$ which is nontrivial and has an infinite group of twists $\mathcal{T}\left(T^{\prime}\right)$

(3) $T$ has a collapse $T^{\prime}$ which is nontrivial and invariant under $\operatorname{Out}(\mathcal{D}(T))$.

$\mathcal{D}(T)$ denotes the deformation space of $T$ over groups of cardinality $\leq k$. All reduced trees in $\mathcal{D}(T)$ have edge stabilizers of order $k$ (see Subsection 2.3). Also note that $T_{c}$ is invariant under $\operatorname{Out}(\mathcal{D}(T))$, and dominated by $T$, so we get:

Corollary 7.11. $T$ has a collapse which is nontrivial and has an infinite group of twists, or $T$ dominates a nontrivial tree $T^{\prime}$ with finite edge stabilizers which is invariant under $\operatorname{Out}(\mathcal{D}(T))$.

Proof of Proposition 7.10. We can assume that $T$ is reduced. We first consider the case when $T_{c}$ is trivial. Since edges of $T$ belong to the same cylinder if and only if they have the same stabilizer, all edges of $T$ have the same stabilizer, a finite normal subgroup $A$.

If there is only one orbit of edges, $T$ is the unique reduced tree in $\mathcal{D}(T)$ because no edge stabilizer may be properly contained in another [46]. It follows that $T^{\prime}=$ $T$ is invariant under $\operatorname{Out}(\mathcal{D}(T))$.

Assume that there is more than one orbit of edges. Since $A$ is normal and finite, its centralizer in $G$ has finite index, so for any vertex $v$ in an arbitrary collapse $T^{\prime}$ of $T$ (including $T$ itself), and any edge $e$ of $T^{\prime}$ incident to $v$, the group $Z_{G_{v}}\left(G_{e}\right)$ is infinite as soon as $G_{v}$ is infinite. By Lemma 2.13, if Assertion 2 does not hold, then all vertex stabilizers of nontrivial collapses $T^{\prime}$ are either finite or infinite with infinite center. Since $T$ is reduced, this is possible only if $T$ has one orbit of vertices, all vertex stabilizers equal to $A$, and only two orbits of edges (this is respectively because a non-trivial amalgam $H_{1} *_{A} H_{2}$, an $\mathrm{HNN}$ extension $H_{1} *_{A}$ with $H_{1} \neq A$, and a double $\mathrm{HNN}$ extension $\left(A *_{A}\right) *_{A}$, have finite center). In particular, $G / A$ is free of rank 2 . One easily checks that collapsing the orbit of any edge gives a tree $T^{\prime}$ with $\mathcal{T}\left(T^{\prime}\right)$ infinite, though Lemma 2.13 does not apply. This concludes the case when $T_{c}$ is trivial.

From now on, we assume that $T_{c}$ is nontrivial and some edge $\varepsilon=(x, Y)$ of $T_{c}$ has infinite stabilizer. View the cylinder $Y$ as a subtree of $T$ containing $x$, and consider an edge $e \subset Y$ with origin $x$. Then we have $G_{Y}=N_{G}\left(G_{e}\right)$ and 
$G_{\varepsilon}=G_{Y} \cap G_{x}=N_{G_{x}}\left(G_{e}\right)$. We know that $Z_{G_{x}}\left(G_{e}\right)$ is infinite, but we cannot apply Lemma 2.13 since we do not know that $Z\left(G_{x}\right)$ is finite.

Assume for a moment that $Y$ contains edges from at least two $G$-orbits. Consider $T^{\prime}$ obtained from $T$ by collapsing all edges in the orbit of $e$, and denote by $x^{\prime}$ the image of $x$ in $T^{\prime}$. Since $T$ is reduced, $G_{x} \varsubsetneqq G_{x^{\prime}}$. By the assumption on $Y$, there is an edge $e^{\prime}$ of $T^{\prime}$ incident to $x^{\prime}$ with $G_{e^{\prime}}=G_{e}$. Since $Z_{G_{x^{\prime}}}\left(G_{e^{\prime}}\right)$ is infinite, we are done if $Z\left(G_{x^{\prime}}\right)$ is finite. We now show that $Z\left(G_{x^{\prime}}\right)$ being infinite leads to a contradiction. Edge stabilizers of $T$ being finite, $x$ is the unique point of $T$ fixed by $G_{\varepsilon}$. Since $G_{\varepsilon} \subset G_{x} \subset G_{x^{\prime}}$, the group $Z\left(G_{x^{\prime}}\right)$ normalizes $G_{\varepsilon}$, hence also fixes $x$, and only $x$ since it is infinite. This implies that $G_{x^{\prime}}$ fixes $x$, a contradiction to $G_{x} \varsubsetneqq G_{x^{\prime}}$.

Returning to the general case, there is a $G$-invariant partition of the set of cylinders: those for which there is an edge $(x, Y)$ of $T_{c}$ with infinite stabilizer, and the others. Thanks to the previous argument, we may assume that all edges contained in a given cylinder of the first type belong to the same $G$-orbit. Let now $T^{\prime}$ be the tree obtained from $T$ by collapsing all edges in cylinders of the second type. It is nontrivial (but $T^{\prime}=T$ is possible). We show that $T^{\prime}$ does not change if we replace $T$ by another reduced tree $T_{1}$ in $\mathcal{D}(T)$. This implies that $T^{\prime}$ is invariant under $\operatorname{Out}(\mathcal{D}(T))$.

One may join $T$ and $T_{1}$ by slide moves (see Subsection 2.3). In a slide move, an edge $e$ slides over an edge $f$ belonging to a different orbit, with $G_{e} \subset G_{f}$. Here one must have $G_{e}=G_{f}$, so $e$ and $f$ belong to the same cylinder, necessarily of the second type. The slide move does not change $T^{\prime}$ since the cylinder gets collapsed.

Proof of Theorem 7.6. We prove the "only if " direction (the other direction is clear since twists act by conjugations on vertex groups). We may assume that all groups in $Q$ are infinite. We first suppose that $G$ is one-ended relative to $Q$. Let $T$ be the canonical elementary JSJ tree relative to $Q=\mathcal{P} \cup \mathcal{H}$ as in Subsection 3.3. Its edge stabilizers are finitely generated by Lemma 3.7. By Theorem 4.6, if $\operatorname{Out}\left(G ; Q^{(t)}\right)$ is infinite, then either $\mathcal{T}(T)$ is infinite and we are done, or $T$ has at least a non-rigid $\mathrm{QH}$ vertex $v$ with $\operatorname{Out}\left(G_{v} ; \mathcal{B}_{v}\right)$ infinite.

The underlying orbifold group $\pi_{1}(\Sigma)$ splits, relative to its boundary subgroups, over a maximal virtually cyclic subgroup with infinite center (see [17], Proposition 3.1). This induces an elementary splitting of $G_{v}$ which extends to a splitting of $G$. By Lemma 2.6, this splitting of $G$ is relative to $\mathcal{Q}$ (because the intersection of $G_{v}$ with a conjugate of a group in $Q$ projects into a boundary subgroup in $\pi_{1}(\Sigma)$ ), and has an infinite group of twists by Lemmas 7.9 and 2.14. This proves the theorem if $G$ is one-ended relative to $Q$. 
In the general case, let $k \in \mathbb{N}$ be the smallest number such that $G$ splits relative to $Q$ over a group of cardinality $k$. Let $T$ be a reduced JSJ tree relative to $Q$ over subgroups of cardinality $k$ (see Subsection 2.5). Its deformation space is invariant under $\operatorname{Out}\left(G ; \mathrm{Q}^{(\mathrm{t})}\right)$. By Corollary 7.11, either some collapse of $T$ has an infinite group of twists (and we are done), or $T$ dominates a nontrivial tree $T^{\prime}$ with finite edge stabilizers which is invariant under $\operatorname{Out}\left(G ; Q^{(t)}\right)$. Note that the groups in $\mathcal{Q}$ are elliptic in $T^{\prime}$, since they are elliptic in $T$ and $T$ dominates $T^{\prime}$.

We may assume that $\mathcal{T}\left(T^{\prime}\right)$ is finite. Let $A_{0} \subset \operatorname{Out}\left(G ; Q^{(t)}\right)$ be the finite index subgroup acting trivially on the graph $T^{\prime} / G$. By Assertion 1 of Lemma 2.12, there exists a vertex group $G_{v}$ of $T^{\prime}$ such that $\rho_{v}\left(A_{0}\right) \subset \operatorname{Out}\left(G_{v}\right)$ is infinite. In particular, $G_{v}$ is infinite.

The group $G_{v}$ is hyperbolic relative to the family $\mathcal{P}_{\mid G_{v}}$ (see Lemma 3.5). By Lemma 2.10, we have $\rho_{v}\left(A_{0}\right) \subset \operatorname{Out}\left(G_{v} ; Q_{\mid G_{v}}^{(\mathrm{t})}\right)$.

If the theorem holds for $G_{v}$, we get a graph of groups decomposition $\Gamma_{0}$ of $G_{v}$ relative to $\mathcal{Q}_{\mid G_{v}}$ having an infinite group of twists. Since $T^{\prime}$ has finite edge stabilizers, $\Gamma_{0}$ is relative to Inc $v$ and one may refine $T^{\prime} / G$ to a graph of groups $\Lambda$ by using $\Gamma_{0}$. By Lemma 2.14, the splitting $\Lambda$ has an infinite group of twists. It is relative to $Q$ by Lemma 2.6.

If the theorem does not hold for $G_{v}$, we repeat the construction. If the process stops after finitely many steps, we get a splitting $\Lambda$ as in the previous case. If it does not stop, we get an infinite sequence of trees $T_{i}$ relative to $Q$ with finite edge stabilizers, with $T_{i+1}$ strictly dominating $T_{i}$. Since $G$ is finitely presented relative to $Q$, there is a Stallings-Dunwoody decomposition relative to $\mathcal{Q}$ (see Subsection 2.5), and we reach a contradiction (see [19, p. 130]).

7.3. Infinity of unmarked automorphisms. Using Theorem 6.2 , we now characterize relatively hyperbolic groups for which $\operatorname{Out}(G ; \mathcal{P})$ is infinite.

We first note the following consequence of Theorem 7.6.

Corollary 7.12. Let $G$ be hyperbolic relative to $\mathcal{P}=\left\{P_{1}, \ldots, P_{n}\right\}$, where each $P_{i}$ is infinite and finitely generated, and let $\mathcal{H}$ be a finite family of finitely generated subgroups.

If $\operatorname{Out}\left(G ; \mathcal{P}, \mathcal{H}^{(\mathrm{t})}\right)$ is infinite, there is an elementary splitting relative to $\mathcal{P} \cup \mathcal{H}$ with an infinite group of twists, or there is an i such that the natural map

$$
\operatorname{Out}\left(G ; \mathcal{P}, \mathcal{H}^{(\mathrm{t})}\right) \longrightarrow \operatorname{Out}\left(P_{i}\right)
$$

(defined because $P_{i}$ equals its normalizer) has infinite image. 
Proof. If all maps $\operatorname{Out}\left(G ; \mathcal{P}, \mathcal{H}^{(\mathrm{t})}\right) \rightarrow \operatorname{Out}\left(P_{i}\right)$ have finite image, then the intersection of their kernels, namely $\operatorname{Out}\left(G ; \mathcal{P}^{(\mathrm{t})}, \mathcal{H}^{(\mathrm{t})}\right)$, is infinite. We can now apply Theorem 7.6.

Corollary 7.13. Assume furthermore that $G$ is non-elementary (i.e. $P_{i} \neq G$ ). Then $\operatorname{Out}\left(G ; \mathcal{P}, \mathcal{H}^{(\mathrm{t})}\right)$ is infinite if and only if $G$ has an elementary splitting as a graph of groups $\Lambda$ relative to $\mathcal{P} \cup \mathcal{H}$ such that one of the following holds:

- the group of twists of $\Lambda$ is infinite,

- or $\Lambda$ has a vertex $v$ such that $G_{v}=P_{i}$ is a maximal parabolic subgroup and $\operatorname{Out}\left(G_{v} ; \operatorname{Inc}_{v}^{(\mathrm{t})}, \mathcal{H}_{\mid G_{v}}^{(\mathrm{t})}\right)$ is infinite.

Proof. As in Theorem 7.6, the "if" direction is clear, so we can assume that $\operatorname{Out}\left(G ; \mathcal{P}, \mathcal{H}^{(\mathrm{t})}\right)$ is infinite. By Corollary 7.12, and up to renumbering, we can assume that $\operatorname{Out}\left(P_{1} \subsetneq\left(G ; \mathcal{P}, \mathcal{H}^{(\mathrm{t})}\right)\right)$ is infinite. By Theorem 6.2, $P_{1}$ is a vertex group in some elementary decomposition of $G$ relative to $\mathcal{P} \cup \mathcal{H}$ and $\operatorname{Out}\left(P_{1} ; \operatorname{Inc}_{v}^{(\mathrm{t})}, \mathcal{H}_{\mid G_{v}}^{(\mathrm{t})}\right)$ has finite index $\operatorname{in} \operatorname{Out}\left(P_{1} \subsetneq\left(G ; \mathcal{P}, \mathcal{H}^{(\mathrm{t})}\right)\right)$. In particular, $\operatorname{Out}\left(P_{1} ; \operatorname{Inc}_{v}^{(\mathrm{t})}, \mathcal{H}_{\mid G_{v}}^{(\mathrm{t})}\right)$ is infinite.

7.4. Hyperbolic groups. We apply the results of the previous subsections to the case when $G$ is a hyperbolic group. We say that a subgroup of $G$ is $z_{\max }$ if it is maximal for inclusion among virtually cyclic subgroups with infinite center. Note that any virtually cyclic subgroup $C$ with infinite center is contained in a unique $Z_{\max }$ subgroup $\widehat{C}$ (the pointwise stabilizer of $\partial C$ ).

Given any splitting of a hyperbolic group over a $z_{\max }$ subgroup $C$, any central element $c \in C$ of infinite order defines a twist of infinite order in $\operatorname{Out}(G)$.

Theorem 7.14. If $G$ is hyperbolic, $\operatorname{Out}(G)$ is infinite if and only if there is a nontrivial splitting of $G$ over a $Z_{\max }$ subgroup (such a splitting always has a twist of infinite order).

If $\mathcal{H}$ is a finite family of finitely generated subgroups, $\operatorname{Out}\left(G ; \mathcal{H}^{(\mathrm{t})}\right)$ is infinite if and only if there is a non-trivial splitting over a $Z_{\max }$ subgroup relative to $\mathcal{H}$.

It was proved independently by M. Carette [10] that $\operatorname{Out}(G)$ is infinite if and only if $G$ has a splitting over a finite group or a (maybe non maximal) virtually cyclic group with infinite center, with a twist of infinite order (see $[45,17]$ for the one-ended case).

Proof. The "if" direction is clear, so we assume that $\operatorname{Out}\left(G ; \mathcal{H}^{(\mathrm{t})}\right)$ is infinite. All splittings considered in this proof will be relative to $\mathcal{H}$. 
Theorem 7.6 and Lemma 2.15 say that $G$ has a one-edge splitting over a (possibly finite) virtually cyclic group $C$, whose group of twists is infinite. We assume that this splitting is an amalgam $G=A *_{C} B$; the case of an HNN extension is similar. We first explain how to replace this amalgam over $C$ by one over a (possibly non-maximal) virtually cyclic subgroup $C^{\prime}$ with infinite center.

If $C$ is infinite with finite center, its centralizer in $G$ is finite and this forces the group of twists to be finite. So assume that $C$ is finite.

If both $Z_{A}(C) / Z(A)$ and $Z_{B}(C) / Z(B)$ are finite, the group of twists is finite, so assume for instance that $Z_{A}(C) / Z(A)$ is infinite. Note that $Z(A)$ has to be finite, since otherwise $A$ would be virtually cyclic, and $Z_{A}(C) / Z(A)$ would be finite. Consider an element of infinite order $t \in Z_{A}(C)$, and perform a fold to get $G=A *\langle C, t\rangle\langle B, t\rangle$. This is a splitting over a virtually cyclic subgroup $C^{\prime}$ with infinite center, and it is relative to $\mathcal{H}$. The twist defined by $t$ has infinite order in $\operatorname{Out}(G)$ by Lemma 7.9.

Now suppose that an amalgam $G=A^{\prime} *_{C^{\prime}} B^{\prime}$ has an infinite group of twists, with $C^{\prime}$ virtually cyclic with infinite center. Then $A^{\prime}, B^{\prime}$ have finite center. The $Z_{\max }$ subgroup $\widehat{C}^{\prime}$ containing $C^{\prime}$ is elliptic in the amalgam, and one can perform a fold to get an amalgam over $\widehat{C}^{\prime}$. This splitting is non-trivial because $\widehat{C}^{\prime}$ is not conjugate to $A^{\prime}$ or $B^{\prime}$ since they have finite center. The group of twists of the new splitting is clearly infinite.

Theorem 7.15. There is an algorithm which, given a hyperbolic group $G$, decides whether $\operatorname{Out}(G)$ is infinite or not. More generally, if $\mathcal{H}$ is a finite family of finitely generated subgroups, one may decide whether $\operatorname{Out}\left(G ; \mathcal{H}^{(\mathrm{t})}\right)$ is infinite.

Proof. We start with the first assertion.

We first construct an algorithm that stops if and only if $\operatorname{Out}(G)$ is finite. By Theorem 8.1 of [17], one can compute a finite generating set of $\operatorname{Out}(G)$. Moreover, one can solve the word problem in $\operatorname{Out}(G)$ as this amounts to solving (uniformly) the simultaneous conjugacy problem in $G$ (see [8] for a solution). Thus, for each $R>0$, one can determine the ball $B_{R}$ of radius $R$ in the Cayley graph of $\operatorname{Out}(G)$. Checking whether $B_{R}=B_{R+1}$ for some $R$ gives the required algorithm.

It now suffices to construct an algorithm that stops if $\operatorname{Out}(G)$ is infinite. By [17, Lemma 2.8], one can decide whether a subgroup of $G$ (given by generators) is $z_{\max }$ or not. One can therefore enumerate all decompositions of $G$ as an amalgam or HNN extension over $z_{\max }$ subgroups. By Corollary 7.14, this provides an algorithm that stops if $\operatorname{Out}(G)$ is infinite.

The argument to decide whether $\operatorname{Out}\left(G ; \mathcal{H}^{(\mathrm{t})}\right)$ is infinite is similar. The first algorithm is the same since Theorem 8.1 of [17] provides generators for $\operatorname{Out}\left(G ; \mathcal{H}^{(\mathrm{t})}\right)$. 
For the second algorithm, one has to restrict to splittings relative to $\mathcal{H}$, so one needs an algorithm that, given a splitting, stops if the splitting is relative to $\mathcal{H}$. This is done by choosing a generating set $S_{i}$ for each $H_{i} \in \mathcal{H}$, enumerating all conjugates of $S_{i}$, and comparing them with words written using the generators of a vertex group.

In general, we do not know how to decide whether $\operatorname{Out}(G ; \mathcal{H}$ ) is infinite (see Remark 4.8). The following is an answer when $G$ is hyperbolic relative to $\mathcal{H}$.

Proposition 7.16. There is an algorithm which, given a torsion-free hyperbolic group $G$, a finite family $\mathcal{P}$ of finitely generated locally quasiconvex subgroups $P_{i}$ such that $G$ is hyperbolic relative to $\mathcal{P}$, and a finite family $\mathcal{H}$ of finitely generated subgroups, decides whether $\operatorname{Out}\left(G ; \mathcal{P}, \mathcal{H}^{(\mathrm{t})}\right)$ is infinite.

Since $G$ is assumed to be hyperbolic relative to $\mathcal{P}$, each $P_{i}$ is quasiconvex in $G$. In particular, $P_{i}$ is itself a hyperbolic group. Local quasiconvexity of $P_{i}$ means that its finitely generated subgroups are quasiconvex (in $P_{i}$, hence also in the hyperbolic group $G$ ).

Proof. First, using Touikan's algorithm [63, Theorem A], one can decide whether $G$ splits as a free product relative to $\mathcal{P} \cup \mathcal{H}$. If it does, it is easy to decide whether $\operatorname{Out}\left(G ; \mathcal{P}, \mathcal{H}^{(\mathrm{t})}\right)$ is infinite using Proposition 7.2 and Remark 7.3.

So assume that $G$ is one-ended relative to $\mathcal{P} \cup \mathcal{H}$. We may also assume that no $P_{i}$ is cyclic. We use [63, Theorem C] to decide whether $G$ splits in a suitable way. For this, we need our parabolic groups $P_{i}$ to be algorithmically tractable in the sense of [63, Definition 1.13].

Since $P_{i}$ is locally quasiconvex, it is hyperbolic and the conjugacy problem is solvable in $P_{i}$. Moreover, local quasiconvexity of $P_{i}$ implies that one can decide whether a finite subset $S \subset P_{i}$ generates $P_{i}$ or not, by checking whether a given generating set of $P_{i}$ lies in the quasiconvex subgroup $\langle S\rangle$ [40]. This says that $P_{i}$ is algorithmically tractable.

Applying [63, Theorem C], one can decide whether there exists an elementary splitting of $G$ (viewed as a relatively hyperbolic group) relative to $\mathcal{P} \cup \mathcal{H}$ with finitely generated edge groups, and if so find one. By local quasiconvexity of $P_{i}$, edge groups of the splitting are quasiconvex in the hyperbolic group $G$, and so are vertex groups (see Subsection 3.2).

Iterating this process, one can compute a maximal elementary splitting $\Gamma$ of $G$ relative to $\mathcal{P} \cup \mathcal{H}$ (i.e. a splitting that cannot be refined non-trivially into an elementary splitting relative to $\mathcal{P} \cup \mathcal{H})$. Arguing as in [16, Section 6] and [17, Lemma 2.34], 
one may then recognize the $\mathrm{QH}$ subgroups in $\Gamma$, and find the canonical elementary JSJ decomposition of $G$ relative to $\mathcal{P} \cup \mathcal{H}$ (see also [18, Theorem 3.12]).

By Theorem 4.6, $\operatorname{Out}\left(G ; \mathcal{P}, \mathcal{H}^{(\mathrm{t})}\right)$ is infinite if and only if the group of twists $\mathcal{T}$ is infinite, or there is a vertex $v$ such that $\operatorname{Out}\left(G_{v} ; \operatorname{Inc}_{v}^{(\mathrm{t})}, \mathcal{H}_{\mid G_{v}}^{(\mathrm{t})}\right)$ is infinite.

Recall that $\mathcal{T}$ is isomorphic to a quotient of $\prod_{e \in E} Z_{G_{o(e)}}\left(G_{e}\right)$ by edge and vertex relations. Each group $Z_{G_{o(e)}}\left(G_{e}\right)$ is either trivial or infinite cyclic, and is computable. Edge relations and vertex relations are generated by embeddings of groups $Z\left(G_{e}\right)$ and $Z\left(G_{v}\right)$ in this product. Since one can compute the corresponding subgroups of the abelian group $\prod_{e \in E} Z_{G_{o(e)}}\left(G_{e}\right)$, one can decide whether the group of twists is infinite or not.

To decide whether $\operatorname{Out}\left(G_{v} ; \operatorname{Inc}_{v}^{(\mathrm{t})}, \mathcal{H}_{\mid G_{v}}^{(\mathrm{t})}\right)$ is infinite, we can apply [16] (or more explicitly [17, Corollary 3.4]), or Theorem 7.15, but we need to determine $\mathcal{H}_{\mid G_{v}}$ (see Definition 2.3). Given $H \in \mathcal{H}$ generated by a finite set $S$, one can decide whether there is $g \in G$ such that $S^{g} \subset G_{v}$ in the same way as above because $G_{v}$ is quasiconvex. One can similarly decide whether $H$ fixes an edge, which allows to compute $\mathcal{H}_{\mid G_{v}}$.

\section{Fixed subgroups}

In this section, we use JSJ decompositions to study fixed subgroups of automorphisms. This is inspired by arguments due to Sela [58]. The proof of the Scott conjecture (Theorem 8.1) given below is not really new, but using the relative JSJ decomposition makes the argument more direct. Using Theorem 8.2, we will prove in [37] that, given a toral relatively hyperbolic group $G$, there are only finitely many possibilities for fixed subgroups of automorphisms of $G$, up to isomorphism. This was proved by Shor [62] for $G$ torsion-free hyperbolic.

Theorem 8.1 ([4]). Let $\alpha$ be an automorphism of a free group $F_{n}$. Its fixed subgroup Fix $\alpha$ has rank at most $n$.

Proof. The smallest free factor containing Fix $\alpha$ is $\alpha$-invariant. Replacing $G$ by this free factor, we may assume that $F_{n}$ is one-ended (freely indecomposable) relative to Fix $\alpha$. We also assume that Fix $\alpha$ is not cyclic.

Let $T$ be the canonical cyclic JSJ tree relative to Fix $\alpha$ (see Subsection 3.3), and let $G_{v}$ be the vertex stabilizer containing Fix $\alpha$. It is $\alpha$-invariant because $T$ is invariant and $v$ is the only vertex fixed by Fix $\alpha$, and abelianizing shows that it has rank $\leq n$. By Remark 2.9 it cannot be flexible QH because Fix $\alpha$ is not cyclic, so it is rigid. By standard arguments due to Paulin and Rips, $\alpha$ has finite 
order in $\operatorname{Out}\left(G_{v}\right)$ : otherwise, applying Theorem 3.9 with $\mathcal{P}$ consisting of incident edge groups and $\mathcal{H}$ consisting of Fix $\alpha$ (which is finitely generated) yields a cyclic splitting of $G_{v}$ which contradicts rigidity. By Dyer-Scott [23], Fix $\alpha$ is a free factor of $G_{v}$ so has rank $\leq n$.

If we do not wish to use Gersten's result that Fix $\alpha$ is finitely generated [27], we argue by contradiction as follows. Let $H$ be a finitely generated free factor of Fix $\alpha$ of rank $>n$. We claim that $F_{n}$ is one-ended relative to $H$ (see [55] and Lemma 7.6 of [36] for more general statements). Otherwise, let $\hat{H}$ be the smallest free factor of $F_{n}$ containing $H$. Then $\hat{H}$ is $\alpha$-invariant, and Fix $\alpha \cap \widehat{H}$ has rank at most $n-1$ (assuming that the theorem holds in $\hat{H}$ by induction on $n$ ), a contradiction since Fix $\alpha \cap \widehat{H}$ retracts onto $H$.

Define $G_{v}$ as above, using the cyclic JSJ splitting of $F_{n}$ relative to $H$. The fixed subgroup of $\alpha_{\mid G_{v}}$ is a subgroup of Fix $\alpha$ which has rank $\leq n$ and contains $H$. This is a contradiction since $H$ is a retract of Fix $\alpha$.

This proof uses [23], which is specific to free groups. Applying the same argument to (relatively) hyperbolic groups only yields:

Theorem 8.2. Let $G$ be hyperbolic relative to a finite family $\mathcal{P}$ of slender subgroups. Consider $\alpha \in \operatorname{Aut}(G ; \mathcal{P})$ such that $\operatorname{Fix} \alpha$ is not elementary (i.e. not virtually cyclic or parabolic). Then Fix $\alpha$ is contained in an $\alpha$-invariant vertex group $G_{v}$ of a splitting of $G$ over elementary subgroups relative to $\mathcal{P}$, and $\alpha_{\mid G_{v}}$ has finite order in $\operatorname{Out}\left(G_{v}\right)$.

Proof. The proof is similar to the one above. Note that Fix $\alpha$ is finitely generated by [39, Cor. 9.2] because it is relatively quasiconvex [51] and groups in $\mathcal{P}$ are slender. First assume that $G$ is one-ended relative to $\mathcal{P} \cup\{$ Fix $\alpha\}$. Let $T_{\text {can }}$ be the canonical elementary JSJ decomposition of $G$ relative to $\mathcal{P} \cup\{$ Fix $\alpha\}$. Let $v$ be the unique vertex of $T_{\text {can }}$ fixed by Fix $\alpha$. As above, $G_{v}$ is $\alpha$-invariant; it cannot be QH because it contains the universally elliptic subgroup Fix $\alpha$ which is not virtually cyclic, so $G_{v}$ is rigid: it has no elementary splitting relative to Inc $_{v} \cup \mathcal{P}_{\mid G_{v}} \cup\{$ Fix $\alpha\}$. By Lemma 3.8, $G_{v}$ is hyperbolic relative to Inc $v \cup \mathcal{P}_{\mid G_{v}}$. By Theorem 3.9, the group $\operatorname{Out}\left(G_{v} ; \operatorname{Inc}_{v}, \mathcal{P}_{\mid G_{v}},\{\operatorname{Fix} \alpha\}^{(\mathrm{t})}\right)$ is finite. It contains the class of $\alpha_{\mid G_{v}}$ by Lemma 2.10, so $\alpha_{\mid G_{v}}$ has finite order in $\operatorname{Out}\left(G_{v}\right)$.

If $G$ is not relatively one-ended, we consider a reduced Stallings-Dunwoody tree $S$ relative to $\mathcal{P} \cup\{$ Fix $\alpha\}$ (see Subsection 2.5). Since Fix $\alpha$ is infinite, it fixes a unique vertex $u \in S$. The deformation space of $S$ is $\alpha$-invariant, so $\alpha\left(G_{u}\right)$ fixes a vertex $u^{\prime} \in S$. Since Fix $\alpha \subset \alpha\left(G_{u}\right)$ fixes only $u$, we have $u^{\prime}=u$ and $\alpha\left(G_{u}\right)=G_{u}$. We now apply the previous analysis to the restriction of $\alpha$ to $G_{u}$, 
which is hyperbolic relative to $\mathcal{P}_{\mid G_{u}}$ by Lemma 3.5. We get a splitting $\Lambda$ of $G_{u}$ relative to $\mathcal{P}_{\mid G_{u}}$, and we obtain the desired splitting of $G$ by refining $S / G$ using $\Lambda$ (see Lemma 2.6).

\section{Rigid groups have finitely many automorphisms}

The goal of this section is to prove Theorem 3.9. Let us first recall its statement.

Theorem 3.9. Let $G$ be hyperbolic relative to finitely generated subgroups $\mathcal{P}=\left\{P_{1}, \ldots, P_{n}\right\}$, with $P_{i} \neq G$. Let $\mathcal{H}=\left\{H_{1}, \ldots, H_{q}\right\}$ be another family of finitely generated subgroups. If $\operatorname{Out}\left(G ; \mathcal{P}, \mathcal{H}^{(\mathrm{t})}\right)$ is infinite, then $G$ splits over an elementary subgroup relative to $\mathcal{P} \cup \mathcal{H}$.

As mentioned earlier, the proof uses $\mathbb{R}$-trees. All actions on $\mathbb{R}$-trees considered here are by isometries. An arc is a subset isometric to an interval $[a, b] \subset \mathbb{R}$ with $a \neq b$. As in the simplicial case, an action on an $\mathbb{R}$-tree $T$ is relative to subgroups $H_{i}$ if each $H_{i}$ is elliptic (fixes a point) in $T$.

Because the parabolic groups are not assumed to be slender, we will need to analyze actions on $\mathbb{R}$-trees which are not quite stable.

\subsection{Constructing an R-tree}

Theorem 9.1 ([1]). Let $G, \mathcal{P}, \mathcal{H}$ be as in Theorem 3.9. If $\operatorname{Out}\left(G ; \mathcal{P}, \mathcal{H}^{(\mathrm{t})}\right)$ is infinite, then $G$ has a non-trivial action on an $\mathbb{R}$-tree $T$ relative to $\mathcal{P} \cup \mathcal{H}$ such that arc stabilizers are elementary.

The proof is essentially in [1], noting that a locally elementary subgroup is elementary by Lemma 3.1. We also add the remark that the groups $P_{i}, H_{j}$ are elliptic in $T$.

Proof. Let $\varphi_{k}$ be automorphisms representing distinct elements of $\operatorname{Out}\left(G ; \mathcal{P}, \mathcal{H}^{(\mathrm{t})}\right)$. Let $X$ be a $\delta$-hyperbolic space on which $G$ acts as in Subsection 3.1. Consider a finite generating set $S$ of $G$, and the minimal displacement

$$
d_{k}=\inf _{x \in X} \max _{s \in S} d_{X}\left(x, \varphi_{k}(s) \cdot x\right) .
$$

Choose a point $x_{k} \in X$ where $\max _{s \in S} d_{X}\left(x_{k}, \varphi_{k}(s) . x_{k}\right) \leq d_{k}+\frac{1}{k}$.

Using the Bestvina-Paulin method, it is shown in [1] that $d_{k}$ goes to infinity, the rescaled pointed metric spaces $X_{k}=\left(\frac{1}{d_{k}} X, x_{k}\right)$ converge to an R-tree $T$ (after taking a subsequence), and the action of $G$ on $X_{k}$ twisted by $\varphi_{k}$ converges to a 
non-trivial isometric action of $G$ on $T$ with locally elementary (hence elementary) arc stabilizers.

We now prove that the action is relative to $\mathcal{P} \cup \mathcal{H}$. Since groups in $\mathcal{P} \cup \mathcal{H}$ are finitely generated, it suffices to show that any element $g$ belonging to $P_{i}$ or $H_{j}$ is elliptic in $T$.

Suppose $g$ acts hyperbolically in $T$. Then there exists $a \in T$ such that

$$
d_{T}\left(a, g^{2} a\right)=2 d_{T}(a, g a)>0 .
$$

If $a_{k}$ is an approximation point of $a$ in $X_{k}=\frac{1}{d_{k}} X$, then

$$
\frac{d_{X}\left(a_{k}, \varphi_{k}\left(g^{2}\right) a_{k}\right)-d_{X}\left(a_{k}, \varphi_{k}(g) a_{k}\right)}{d_{k}}
$$

converges to

$$
d_{T}\left(a, g^{2} a\right)-d_{T}(a, g a)=d_{T}(a, g a)>0,
$$

so for $k$ large enough

$$
d_{X}\left(a_{k}, \varphi_{k}\left(g^{2}\right) a_{k}\right)-d_{X}\left(a_{k}, \varphi_{k}(g) a_{k}\right)>2 \delta+\frac{d_{k}}{2} d_{T}(a, g a) .
$$

Lemme 9.2.2 of [12] implies that $\varphi_{k}(g)$ acts loxodromically on $X$, with translation length going to infinity since $d_{k} \rightarrow \infty$. This is a contradiction if $g \in P_{i}$, since every $\varphi_{k}(g)$ is parabolic in this case. If $g \in H_{j}$, all elements $\varphi_{k}(g)$ are conjugate so have the same translation length in $X$, also a contradiction.

Remark 9.2. One can show that a group $H \in \mathcal{H}$ is elliptic in $T$, even if it is not assumed to be finitely generated. We know that every $h \in H$ is elliptic. If $H$ is not elliptic, it fixes an end of $T$, so every finitely generated subgroup of $H$ fixes a ray. This implies that finitely generated subgroups of $H$ are parabolic, so $H$ is parabolic and therefore elliptic in $T$.

On the other hand, Theorem 9.4 below requires finite generation.

Remark 9.3. The hypothesis that automorphisms act trivially on $H_{j}$ may be weakened. It is sufficient to assume that their growth under iteration is slower on $H_{j}$ than on $G$.

9.2. Hypostability. To deduce a splitting as in Theorem 3.9 from the action on the R-tree of Theorem 9.1, we will generalize the following basic fact (see Theorem 9.9): 
Theorem 9.4 ([3, Thm 9.6]). Let $G$ be a finitely presented group, and let $Q$ be a finite family of finitely generated subgroups. Assume that $G$ has a non-trivial stable action on an $\mathbb{R}$-tree $T$ relative to $Q$. Then $G$ splits relative to $Q$ over a group $K$ which is an extension $1 \rightarrow A \rightarrow K \rightarrow \mathbb{Z}^{k} \rightarrow 1$, where A fixes an arc of $T$ and $k \geq 0$.

Recall that an arc $J$ is stable if any subarc of $J$ has the same stabilizer as $J$. An action is stable if every arc $I$ contains a stable subarc $J$.

Corollary 9.5. Theorem 3.9 holds if every $P_{i}$ is slender and $G$ is finitely presented.

Proof. Assume that every $P_{i}$ is slender. In this case a subgroup of $G$ is elementary if and only if it is slender. In particular, elementary subgroups satisfy the ascending chain condition, so the action on the $\mathbb{R}$-tree $T$ provided by Theorem 9.1 is stable. If furthermore $G$ is finitely presented, Theorem 9.4 (applied with $Q=\mathcal{P} \cup \mathcal{H}$ ) gives a splitting that satisfies the conclusion of Theorem 3.9 (note that $K$ is slender because $A$ is).

In general, however, $G$ is only finitely presented relative to $\mathcal{P}$, and the action on $T$ only satisfies a weaker property than stability, which we call hypostability (see [42] for a different property called semistability).

Definition 9.6. Let $G$ be a group acting on an $\mathbb{R}$-tree $T$. The action is hypostable if, for each arc $I \subset T$, there exists a subarc $J \subset I$ satisfying the following hypostability condition: if $g \in G$ acts hyperbolically in $T$ and $g J \cap J$ is an arc, then $\operatorname{Stab} J=\operatorname{Stab}(g J)$ (equivalently, $g$ normalizes $\operatorname{Stab} J$ ).

Hypostability is weaker than stability, because any stable arc $J$ satisfies the hypostability condition: if $g J \cap J$ is an $\operatorname{arc},\langle\operatorname{Stab}(J), \operatorname{Stab}(g J)\rangle$ is contained in the stabilizer of $g J \cap J$, which coincides with $\operatorname{Stab}(J)$ and $\operatorname{Stab}(g J)$ by stability of $J$ and $g J$.

Lemma 9.7. Let $G$ be hyperbolic relative to finitely generated subgroups $\mathcal{P}=\left\{P_{1}, \ldots, P_{n}\right\}$. Any action of $G$ on an $\mathbb{R}$-tree $T$ relative to $\mathcal{P}$ with elementary arc stabilizers is hypostable.

Proof. Let $C$ be such that any elementary subgroup $H$ of $G$ of cardinality $>C$ is contained in a unique maximal elementary subgroup $E(H)$ (see Lemma 3.1). Let $I \subset T$ be an arc. If the stabilizer of every subarc has cardinality at most $C$, then a subarc $J \subset I$ whose stabilizer has the greatest cardinality is stable and we are done. 
Otherwise, consider $J \subset I$ whose stabilizer $H=\operatorname{Stab}(J)$ has cardinality greater than $C$. The stabilizer of every subarc of $J$ is elementary, so is contained in $E(H)$. If subgroups of $E(H)$ satisfy the ascending chain condition, in particular if $E(H)$ is virtually cyclic, then $J$ contains a stable subarc. Thus we can assume that $E(H)$ is parabolic.

We prove hypostability by showing that any $g$ such that $g J \cap J$ contains an arc is elliptic in $T$. Indeed, $\left\langle H, H^{g}\right\rangle$ fixes an arc in $T$, so is elementary. It follows that $\left\langle H, H^{g}\right\rangle \subset E(H)$, so $E(H)=E\left(H^{g}\right)=E(H)^{g}$. Since $E(H)$ is its own normalizer, we get $g \in E(H)$. But $T$ is relative to $\mathcal{P}$, so $g$ is elliptic.

Example 9.8. We sketch the construction of an action as in Lemma 9.7 which is not stable. Let $G$ be the free product $G=P * \mathbb{Z}$, with $P$ a (non-slender) finitely generated group containing a copy of the free abelian group on a countable basis $\mathbb{Z}^{(Q)}$. Note that $G$ is hyperbolic relative to $\{P, \mathbb{Z}\}$. Informally, identifying the edge of the free product with $[0,1]$, one can produce an $\mathbb{R}$-tree from the Bass-Serre tree of this splitting by folding the group $\mathbb{Z}^{\left(\left[0, \frac{p}{q}\right] \cap Q\right)}$ on a length $\frac{p}{q}$ for all $0<\frac{p}{q}<1$. The stabilizer of an $\operatorname{arc}[a, b] \subset[0,1]$ is then $\mathbb{Z}^{([0, b] \cap Q)}$. This action is hypostable but unstable.

Theorem 9.9. Theorem 9.4 holds if the action on the $\mathbb{R}$-tree is only assumed to be hypostable, and the group $G$ is only assumed to be finitely presented relative to $Q$.

The proof will be given in the next subsection. The following corollary is an immediate consequence of Lemma 9.7 and Theorem 9.9.

Corollary 9.10. Let $G$ be a relatively hyperbolic group, with $\mathcal{P}, \mathcal{H}$ as in Theorem 3.9. If $G$ acts non-trivially on an $\mathbb{R}$-tree $T$ relative to $\mathcal{P} \cup \mathcal{H}$ with elementary arc stabilizers, then $G$ splits over an elementary subgroup relative to $\mathcal{P} \cup \mathcal{H}$.

Theorem 3.9 follows immediately from this corollary, using the $\mathbb{R}$-tree provided by Theorem 9.1. A refinement of Corollary 9.10 will be given in Subsection 9.4.

Remark 9.11. Let $G$ and $T$ be as in Theorem 9.4. If $T$ is not a line, one can get a splitting over a group $K$ which is an extension of $\mathbb{Z}$ or $\mathbb{Z} / 2 \mathbb{Z}$ by a group $A$ fixing an arc in $T$ (see [3]). One can also approximate $T$ (in the equivariant Gromov topology) by simplicial trees with controlled edge stabilizers, as in [29]. The same facts are true under the assumptions of Theorem 9.9. 
9.3. Proof of Theorem 9.9. Recall that a subtree $Y \subset T$ is indecomposable [31] if, given $\operatorname{arcs} I, L \subset Y$, there exist $g_{1}, \ldots, g_{n} \in G$ such that $L \subset g_{1} I \cup \cdots \cup g_{n} I$, and every $g_{i} I \cap g_{i+1} I$ is an arc. We call $g_{1}, \ldots, g_{n}$ an $I$-covering of $L$.

Lemma 9.12. Assume that $Y \subset T$ is an indecomposable subtree. Given two arcs $I, L \subset Y$ with $I \subset L$, there exists an $I$-covering $g_{1}, \ldots, g_{r}$ of $L$ such that $g_{1}=1$ and every $g_{i} g_{i-1}^{-1}$ is hyperbolic in $T$.

Proof. Given any $I$-covering of $L$, there exists $i$ such that $I \cap g_{i} I$ is an arc, and therefore $1, g_{i}, g_{i-1}, \ldots, g_{1}, g_{2}, \ldots, g_{n}$ is an $I$-covering starting with 1 . From now on, we only consider coverings starting with 1 . The interval covered will always be $L$.

We fix an orientation of $I$. It induces an orientation of $g I$ for any $g \in G$. If $I \cap g I$ is an arc, the orientations of $I$ and $g I$ may agree or disagree on this arc. We first claim that there exists an $I$-covering $1=a_{1}, \ldots, a_{p}$ of $L$ such that, for each $i$, the orientation of $a_{i} I$ agrees with that of $a_{i+1} I$ on their intersection (we say that such an $I$-covering is orientation-preserving).

If not, we can find arcs $g I, g^{\prime} I$ whose intersection is an arc on which the orientations disagree. Define $g_{0}=g^{\prime-1} g$ and $J=I \cap g_{0} I$; the orientations of $I$ and $g_{0} I$ disagree on $J$. Now consider a $J$-covering $1=b_{1}, \ldots, b_{p}$ of $L$. It is also an $I$-covering of $L$. Since $J=I \cap g_{0} I$, we get another $I$-covering of $L$ if we replace some of the $b_{i}$ 's by $b_{i} g_{0}$. Starting with $a_{1}=b_{1}=1$, we then define $a_{i}$ inductively as either $b_{i}$ or $b_{i} g_{0}$, making sure that orientations agree. This proves the claim.

Next note that there exists a hyperbolic element $h \in G$ mapping an $\operatorname{arc} J^{\prime} \subset I$ to a different $\operatorname{arc} h\left(J^{\prime}\right) \subset I$ in an orientation-preserving way. To see this, first choose $h_{1}$ mapping an arc $J_{1} \subset I$ to a disjoint arc $J_{2} \subset I$. If orientation is reversed, choose $h_{2}$ mapping an arc $J_{3} \subset J_{2}$ to an arc $J_{4} \subset I$ different from $J_{1}$ and $J_{3}$. Then take $h$ equal to $h_{2}$ or $h_{2} h_{1}$.

We can now conclude. Let $1=a_{1}, \ldots, a_{r}$ be an orientation-preserving $J^{\prime}$-covering of $L$. Since $J^{\prime} \subset I \cap h^{-1} I$, we get another orientation-preserving $I$-covering if we replace some of the $a_{i}$ 's by $a_{i} h^{-1}$. If $a_{i} a_{i-1}^{-1}$ is not hyperbolic, it is the identity on $a_{i-1} J^{\prime} \cap a_{i} J^{\prime}$. We therefore get the required $I$-covering of $L$ by defining $g_{i}$ inductively as $a_{i}$ or $a_{i} h^{-1}$ so that $g_{i} g_{i-1}^{-1}$ is not the identity on $a_{i-1} J^{\prime} \cap a_{i} J^{\prime}$.

Corollary 9.13. Let $T$ be hypostable, and let $Y \subset T$ be an indecomposable subtree. Any element $g \in G$ fixing an arc in $Y$ fixes the whole of $Y$. In particular, any arc in $Y$ is stable. 
Proof. Assume that $g$ fixes an arc $I \subset Y$. Given $x \in Y$, we aim to prove that $g$ fixes $x$. After making $I$ smaller, we can assume that there is an arc $L$ containing $x$ and $I$. Definition 9.6 provides a subarc $J \subset I$ satisfying the hypostability condition. Consider a $J$-covering $1=g_{1}, \ldots, g_{r}$ of $L$ as in Lemma 9.12. Since $g_{i+1} g_{i}^{-1}$, hence also $g_{i}^{-1} g_{i+1}$, is hyperbolic, hypostability of $J$ implies that all $\operatorname{arcs} g_{i} J$ have the same stabilizer. The element $g$ fixes $g_{1} J=J$, so it fixes every $g_{i} J$ and therefore $L$. In particular, $g$ fixes $x$.

Proof of Theorem 9.9. We explain how to adapt the arguments in [3,29].

Let $\mathcal{Q}=\left\{Q_{1}, \ldots, Q_{q}\right\}$. Let $\left\langle S_{i} \mid \mathcal{R}_{i}\right\rangle$ be a presentation of $Q_{i}$, with $S_{i}$ a finite generating set and $\mathcal{R}_{i}$ a possibly infinite set of relators. Let $\langle S \mid \mathcal{R}\rangle$ be a presentation of $G$ such that $S$ is a finite generating set of $G$ containing each $S_{i}$, and $\mathcal{R}$ is the union of $\mathcal{R}_{1} \cup \cdots \cup \mathcal{R}_{q}$ with finitely many additional relators.

Consider a finite subtree $K \subset T$, i.e. the convex hull of finitely many points. We explain how to choose $K$ large enough so as to yield a resolution of $T$ as in [29, Definition 2.2], even though $G$ is only relatively finitely presented.

For each $s \in S$, we consider $K_{s}=K \cap s^{-1} K$ and the restriction $\varphi_{s}: K_{s} \rightarrow s K_{s}$ of $s$ (we may assume that no $K_{s}$ is empty). We then define the suspension $\Sigma$ as the foliated 2-complex obtained by gluing foliated bands $K_{s} \times[0,1]$ to $K$, where we glue $(x, 0)$ to $x$ and $(x, 1)$ to $\varphi_{s}(x)$. Note that $\pi_{1}(\Sigma)$ is naturally identified with the free group on $S$.

Next, we need all relators of $\mathcal{R}$ to be represented by loops contained in leaves of $\Sigma$. Since each $Q_{i}$ fixes a point $p_{i}$ in $T$, and $S$ contains $S_{i}$, requiring that $K$ contains $p_{1}, \ldots, p_{q}$ takes care of $\mathcal{R}_{1} \cup \cdots \cup \mathcal{R}_{q}$. There remain finitely many other relators, and, as in $[3,29]$, one can choose $K$ so that they also are represented by loops contained in leaves.

The complex $\Sigma$ provides a resolution of $T$ in the sense of Definition 2.2 of [29] (as pointed out in [29], the set $\mathcal{C}$ of curves contained in leaves mentioned in Definition 2.2 is not assumed to be finite). Obtaining a resolution is the only place where finite presentation is used in [29].

As for stability, it is used only in Proposition 4.3 of [29] to prove that, if an element fixes an arc in the subtree $T_{\Gamma_{v}} \subset T$ corresponding to a minimal component of $\Sigma$, then it fixes the whole of $T_{\Gamma_{v}}$. By [31, Proposition 1.25], the geometric $\mathbb{R}$-tree dual to a minimal component of $\Sigma$ is indecomposable, and by Lemma 1.19(1) of [31] its image $T_{\Gamma_{v}} \subset T$ is an indecomposable subtree of $T$. Corollary 9.13 then replaces Proposition 4.3 of [29] under our hypostability assumption.

The rest of the argument of [29] applies without modification. As in Proposition 4.1 of [29], the tree dual to $\Sigma$ is a graph of actions on $\mathbb{R}$-trees $T\left(\mathcal{G}^{\prime}\right)$, such that arc stabilizer of the vertex actions lie in the kernel of these actions. Apply- 
ing Propositions 5.2, 7.2 and 8.1 of [29], one can replace these vertex actions by actions on simplicial trees whose stabilizers are abelian modulo the kernel. By Bass-Serre theory, this provides a splitting of $G$ over the extension of an abelian group $A$ by the kernel $K$ of a vertex action. This splitting is relative to $Q$ as in the Reduction Lemma in $[29, \S 4]$.

9.4. $z_{\max }$ splittings. Say that a subgroup of a relatively hyperbolic group is $z_{\max }$ if it is maximal for inclusion among non-parabolic virtually cyclic subgroups with infinite center.

Theorem 9.14. Let $G$ be hyperbolic relative to $\mathcal{P}=\left\{P_{1}, \ldots, P_{n}\right\}$, with $P_{i}$ finitely generated. Let $\mathcal{H}=\left\{H_{1}, \ldots H_{q}\right\}$ be a (possibly empty) family of finitely generated subgroups. Assume that $G$ acts non-trivially on an $\mathbb{R}$-tree $T$ relative to $\mathcal{P} \cup \mathcal{H}$, and that arc stabilizers are either finite, parabolic or $z_{\max }$.

Then $G$ splits relative to $\mathcal{P} \cup \mathcal{H}$ over a finite, parabolic or $\mathcal{Z}_{\max }$ subgroup.

Corollary 9.10 provides a splitting over an elementary group $A$. Here we assume that every loxodromic arc stabilizer of $T$ is $z_{\max }$, and we claim that the same is true for $A$ : if it is loxodromic, then it is $z_{\max }$.

Proof. - Assume first that the foliated 2-complex $\Sigma$ constructed in the proof of Theorem 9.9 has a minimal component $\Sigma_{v}$. Let $G_{v}$ be the image of its fundamental group in $G$, and let $T_{G_{v}} \subset T$ be the corresponding subtree of $T$. In particular, $G_{v}$ is not elliptic in $T$. By [3, Theorem 5.13] or [29, Theorem 3.1], $G_{v}$ is a vertex group in a decomposition of $G$ as a graph of groups $\Gamma$ relative to $\mathcal{P} \cup \mathcal{H}$. All arcs in $T_{G_{v}}$ have the same stabilizer $F$, a normal subgroup of $G_{v}$.

We claim that $F$ is finite. Otherwise, there are two cases. If $F$ is non-parabolic, hence virtually cyclic, it has finite index in its normalizer and therefore in $G_{v}$, so $G_{v}$ is elliptic, a contradiction. If $F$ is infinite and contained in a maximal parabolic group $P$, then almost malnormality of $P$ implies that the normalizer of $F$ is contained in $P$, so $G_{v} \subset P$. Since $P$ is elliptic in $T$, this is also a contradiction. Thus $F$ is finite.

We now distinguish several cases, depending on the nature of the minimal component $\Sigma_{v}$.

First, $\Sigma_{v}$ cannot be a homogeneous (axial, toral) component since $G_{v}$ would then be virtually $\mathbb{Z}^{k}$ for some $k \geq 2$ ([3, Theorem 9.4(2)] or [29, section 5.1]), hence parabolic, contradicting ellipticity of parabolic groups in $T$.

If $\Sigma_{v}$ is an exotic (Levitt, thin) minimal component, one obtains a splitting of $G$ over $F$, and we are done ([29, Proposition 7.2], [3, Theorem 9.4(3)]). 
If $\Sigma_{v}$ is a surface (IET) component, then by [3, Theorem 9.4(1)] or [29, section 8], after performing some moves, one can assume that $\Sigma_{v}$ is a surface with boundary, and $G_{v} / F$ is the fundamental group of a 2-orbifold with conical singularities supporting a measured foliation with dense leaves. Moreover, $G_{v}$ is a $\mathrm{QH}$ vertex group (with fiber $F$ ) of $\Gamma$.

Let $A \subset G_{v}$ be the preimage of the fundamental group of an essential two-sided simple closed curve not bounding a Möbius band, and not boundary parallel. Then $G$ splits over $A$ relative to $\mathcal{P} \cup \mathcal{H}$. We check that $A$ is $z_{\max }$. Clearly, $A$ is virtually cyclic with infinite center, and is maximal among virtually cyclic subgroups of $G_{v}$. Since $G_{v}$ is $\mathrm{QH}$ and $A$ is not conjugate into a boundary subgroup, it easily follows that $A$ is $z_{\max }$ in $G$.

- The remaining case is when $\Sigma$ has no minimal component. In this case, all leaves of $\Sigma$ are finite, and the dual tree $T_{\Sigma}$ is simplicial. Its edge stabilizers fix an $\operatorname{arc}$ in $T$, so we are done if one of these edge stabilizers is finite or parabolic. Otherwise, arc stabilizers of $T_{\Sigma}$ are virtually cyclic with infinite center but may fail to be $z_{\max }$. If this happens, we have to enlarge the finite tree $K$ used to construct $\Sigma$.

By [48], we can find an exhaustion of $T$ by an increasing sequence of finite subtrees $K_{k}$ such that the corresponding dual trees $T_{\Sigma_{k}}$ strongly converge to $T$. We refer to [48] for the definition of strong convergence; we will only use the fact that, if $A$ is a finitely generated group fixing an $\operatorname{arc}$ in $T$, then $A$ fixes an edge in $T_{\Sigma_{k}}$ for large enough $k$.

We can assume that all dual trees $T_{\Sigma_{k}}$ are simplicial, and that their edge stabilizers are infinite and not parabolic. Let $A_{0}$ be an edge stabilizer of $T_{\Sigma_{0}}$. Then $A_{0}$ fixes an $\operatorname{arc} I$ in $T$. By the hypothesis on arc stabilizers of $T$, the stabilizer of $I$ is a $Z_{\max }$ subgroup $A \supset A_{0}$, which fixes an edge $e$ of $T_{\Sigma_{k}}$ for $k$ large enough. Since $G_{e}$ contains $A$ and fixes an arc in $T$, it is equal to $A$, so $T_{\Sigma_{k}}$ provides the desired splitting.

A similar proof yields the following results.

Theorem 9.15. Let $G$ be hyperbolic relative to $\mathcal{P}=\left\{P_{1}, \ldots, P_{n}\right\}$, with $P_{i}$ slender, and let $\mathcal{H}=\left\{H_{1}, \ldots H_{q}\right\}$ be a family of finitely generated subgroups. Assume that $G$ acts non-trivially on an $\mathbb{R}$-tree $T$ relative to $\mathcal{P} \cup \mathcal{H}$, with elementary arc stabilizers.

Then $G$ splits relative to $\mathcal{P} \cup \mathcal{H}$ over a $Z_{\max }$ subgroup or over the stabilizer of an $\operatorname{arc}$ of $T$. 
Corollary 9.16. Let $G$ be a toral relatively hyperbolic group. Consider a nontrivial action of $G$ on an $\mathbb{R}$-tree relative to non-cyclic abelian subgroups. If arc stabilizers are abelian and stable under taking roots, then $G$ splits (relative to noncyclic abelian subgroups) over an abelian subgroup stable under taking roots.

\section{References}

[1] I. Belegradek and A. Szczepański, Endomorphisms of relatively hyperbolic groups. Internat. J. Algebra Comput. 18 (2008), no. 1, 97-110. Zbl 1190.20034 MR 2394723

[2] M. Bestvina and M. Feighn, Bounding the complexity of simplicial group actions on trees. Invent. Math. 103 (1991), no. 3, 449-469. Zbl 0724.20019 MR 1091614

[3] M. Bestvina and M. Feighn, Stable actions of groups on real trees. Invent. Math. 121 (1995), no. 2, 287-321. Zbl 0837.20047 MR 1346208

[4] M. Bestvina and M. Handel, Train tracks and automorphisms of free groups. Ann. of Math. (2) 135 (1992), no. 1, 1-51. Zbl 0757.57004 MR 1147956

[5] B. H. Bowditch, Cut points and canonical splittings of hyperbolic groups. Acta Math. 180 (1998), no. 2, 145-186. Zbl 0911.57001 MR 1638764

[6] B. H. Bowditch, Relatively hyperbolic groups. Internat. J. Algebra Comput. 22 (2012), no. 3, article id. 1250016, 66 pp. Zbl 1259.20052 MR 2922380

[7] M. R. Bridson and A. Haefliger, Metric spaces of non-positive curvature. Grundlehren der Mathematischen Wissenschaften, 319. Springer, Berlin, 1999. Zbl 0988.53001 MR 1744486

[8] M. R. Bridson and J. Howie, Conjugacy of finite subsets in hyperbolic groups. Internat. J. Algebra Comput. 15 (2005), no. 4, 725-756. Zbl 1083.20032 MR 2160576

[9] I. Bumagin, O. Kharlampovich, and A. Miasnikov, The isomorphism problem for finitely generated fully residually free groups. J. Pure Appl. Algebra 208 (2007), no. 3, 961-977. Zbl 1121.20026 MR 2283438

[10] M. Carette, The automorphism group of accessible groups. J. Lond. Math. Soc. (2) 84 (2011), no. 3, 731-748. Zbl 1239.20040 MR 2855799

[11] M. Clay, Deformation spaces of $G$-trees and automorphisms of Baumslag-Solitar groups. Groups Geom. Dyn. 3 (2009), no. 1, 39-69. Zbl 1226.20022 MR 2466020

[12] M. Coornaert, T. Delzant, and A. Papadopoulos, Géométrie et théorie des groupes. Les groupes hyperboliques de Gromov. Lecture Notes in Mathematics, 1441. Springer, Berlin, 1990. Zbl 0727.20018 MR 1075994

[13] M. Culler and K. Vogtmann, Moduli of graphs and automorphisms of free groups. Invent. Math. 84 (1986), no. 1, 91-119. Zbl 0589.20022 MR 0830040 
[14] F. Dahmani, Classifying spaces and boundaries for relatively hyperbolic groups. Proc. London Math. Soc. (3) 86 (2003), no. 3, 666-684. Zbl 1031.20039 MR 1974394

[15] F. Dahmani, Combination of convergence groups. Geom. Topol. 7 (2003), 933-963. Zbl 1037.20042 MR 2026551

[16] F. Dahmani and D. Groves, The isomorphism problem for toral relatively hyperbolic groups. Publ. Math. Inst. Hautes Études Sci. 107 (2008), 211-290. Zbl 1207.20038 MR 2434694

[17] F. Dahmani and V. Guirardel, The isomorphism problem for all hyperbolic groups. Geom. Funct. Anal. 21 (2011), no. 2, 223-300. Zbl 1258.20034 MR 2795509

[18] F. Dahmani and N. Touikan, Isomorphisms using Dehn fillings: the splitting case. Preprint 2013. arXiv:1311.3937 [math.GR]

[19] W. Dicks and M. J. Dunwoody, Groups acting on graphs. Cambridge Studies in Advanced Mathematics, 17. Cambridge University Press, Cambridge, 1989. Zbl 0665.20001 MR 1001965

[20] C. Druțu and M. V. Sapir, Tree-graded spaces and asymptotic cones of groups. Topology 44 (2005), no. 5, 959-1058. With an appendix by D. Osin and M. V. Sapir. Zbl 1101.20025 MR 2153979

[21] C. Druțu and M. V. Sapir, Groups acting on tree-graded spaces and splittings of relatively hyperbolic groups. Adv. Math. 217 (2008), no. 3, 1313-1367. Zbl 1138.20024 MR 2383901

[22] M. J. Dunwoody, The accessibility of finitely presented groups. Invent. Math. 81 (1985), no. 3, 449-457. Zbl 0572.20025 MR 0807066

[23] J. L. Dyer and G. P. Scott, Periodic automorphisms of free groups. Comm. Algebra 3 (1975), 195-201. Zbl 0304.20029 MR 0369529

[24] M. Forester, Deformation and rigidity of simplicial group actions on trees. Geom. Topol. 6 (2002), 219-267. Zbl 1118.20028 MR 1914569

[25] M. Forester, On uniqueness of JSJ decompositions of finitely generated groups. Comment. Math. Helv. 78 (2003), no. 4, 740-751. Zbl 1040.20032 MR 2016693

[26] K. Fujiwara, On the outer automorphism group of a hyperbolic group. Israel J. Math. 131 (2002), 277-284. Zbl 1015.22007 MR 1942313

[27] S. M. Gersten, Fixed points of automorphisms of free groups. Adv. in Math. 64 (1987), no. 1, 51-85. Zbl 0616.20014 MR 0879856

[28] D. Groves and J. F. Manning, Dehn filling in relatively hyperbolic groups. Israel J. Math. 168 (2008), 317-429. Zbl 1211.20038 MR 2448064

[29] V. Guirardel, Approximations of stable actions on R-trees. Comment. Math. Helv. 73 (1998), no. 1, 89-121. Zbl 0979.20026 MR 1610591

[30] V. Guirardel, Limit groups and groups acting freely on $\mathbb{R}^{n}$-trees. Geom. Topol. 8 (2004), 1427-1470. Zbl 1114.20013 MR 2119301 
[31] V. Guirardel, Actions of finitely generated groups on R-trees. Ann. Inst. Fourier (Grenoble) 58 (2008), no. 1, 159-211. Zbl 1187.20020 MR 2401220

[32] V. Guirardel and G. Levitt, Deformation spaces of trees. Groups Geom. Dyn. 1 (2007), no. 2, 135-181. Zbl 1134.20026 MR 2319455

[33] V. Guirardel and G. Levitt, The outer space of a free product. Proc. Lond. Math. Soc. (3) 94 (2007), no. 3, 695-714. Zbl 1168.20011 MR 2325317

[34] V. Guirardel and G. Levitt, Trees of cylinders and canonical splittings. Geom. Topol. 15 (2011), no. 2, 977-1012. Zbl 2821568 MR 1272.20026

[35] V. Guirardel and G. Levitt, JSJ decompositions: definitions, existence and uniqueness. I: The JSJ deformation space. Preprint 2009. arXiv:0911.3173v2 [math.GR]

[36] V. Guirardel and G. Levitt, JSJ decompositions: definitions, existence and uniqueness. II. Compatibility and acylindricity. Preprint 2009. arXiv:1002.4564v2 [math.GR]

[37] V. Guirardel and G. Levitt, McCool groups of toral relatively hyperbolic groups. Preprint 2014. arXiv:1408.0418 [math.GR] To appear in Algebr. Geom. Topol.

[38] M. Hall Jr., Subgroups of finite index in free groups. Canadian J. Math. 1 (1949), 187-190. Zbl 0031.34001 MR 0028836

[39] G. Ch. Hruska, Relative hyperbolicity and relative quasiconvexity for countable groups. Algebr. Geom. Topol. 10 (2010), no. 3, 1807-1856. Zbl 1202.20046 MR 2684983

[40] I. Kapovich, Detecting quasiconvexity: algorithmic aspects. In G. Baumslag, D. Epstein, R. Gilman, H. Short, and Ch. Sims (eds.), Geometric and computational perspectives on infinite groups. (University of Minnesota and Rutgers University 1994) American Mathematical Society, Providence, R.I., 1996, 91-99. Zbl 0859.20026 Zbl 0832.00034 (collection) MR 1364182 MR 1364175 (collection)

[41] I. Kapovich, Quasiconvexity and amalgams. Int. J. Algebra Comput. 7 (1997), no.6, 771-811. Zbl 0912.20031 MR 1482968

[42] M. Kapovich, On sequences of finitely generated discrete groups. In M. Bonk, J. Gilman, H. Masur, Y. Minsky, and M. Wolf (eds.), In the tradition of Ahlfors-Bers. V. (Rutgers University, 2008) American Mathematical Society, Providence, R.I., 2010, 165-184. Zbl 1234.20060 Zbl 1185.30001(collection) MR 2581836 MR 2590591 (collection)

[43] O. Kharlampovich and A. Myasnikov, Effective JSJ decompositions. In A. V. Borovik (ed.), Groups, languages, algorithms. (Baltimore, MD, 2003) American Mathematical Society, Providence, R.I., 2005, 87-212. Zbl 1093.20019 Zbl 1069.20001 (collection) MR 2159316 MR 2159312 (collection)

[44] M. Korkmaz, Mapping class groups of nonorientable surfaces. Geom. Dedicata 89 (2002), 109-133. Zbl 1016.57013 MR 1890954 
[45] G. Levitt, Automorphisms of hyperbolic groups and graphs of groups. Geom. Dedicata 114 (2005), 49-70. Zbl 1107.20030 MR 2174093

[46] G. Levitt, Characterizing rigid simplicial actions on trees. In J. Burillo, S. Cleary, M. Elder, J. Taback, and E. Ventura (eds.), Geometric methods in group theory. (Seville, 2003) American Mathematical Society, Providence, R.I., 2005, 27-33. Zbl 1079.20040 Zbl 1063.20001 (collection) MR 2139674 MR 2138310 (collection)

[47] G. Levitt, On the automorphism group of generalized Baumslag-Solitar groups. Geom. Topol. 11 (2007), 473-515. Zbl 1143.20014 MR 2302496

[48] G. Levitt and F. Paulin, Geometric group actions on trees. Amer. J. Math. 119 (1997), no. 1, 83-102. Zbl 0878.20019 MR 1428059

[49] P. A. Linnell, On accessibility of groups. J. Pure Appl. Algebra 30 (1983), no. 1, 39-46. Zbl 0545.20020 MR 0716233

[50] J. McCool, Some finitely presented subgroups of the automorphism group of a free group. J. Algebra 35 (1975), 205-213. Zbl 0325.20025 MR 0396764

[51] A. Minasyan and D. Osin, Fixed subgroups of automorphisms of relatively hyperbolic groups. Q. J. Math. 63 (2012), no. 3, 695-712. Zbl 1268.20046 MR 2967171

[52] D. Osin, Elementary subgroups of relatively hyperbolic groups and bounded generation. Internat. J. Algebra Comput. 16 (2006), no. 1, 99-118. Zbl 1100.20033 MR 2217644

[53] D. Osin, Relatively hyperbolic groups: intrinsic geometry, algebraic properties, and algorithmic problems. Mem. Amer. Math. Soc. 179 (2006), no. 843. Zbl 1093.20025 MR 2182268

[54] F. Paulin, Outer automorphisms of hyperbolic groups and small actions on $\mathbb{R}$ trees. In R. C. Alperin (ed.), Arboreal group theory. (Berkeley, CA, 1988) Springer, New York, 1991, 331-343. Zbl 0804.57002 Zbl 0744.00026 (collection) MR 1105339 MR 1105327 (collection)

[55] C. Perin, Elementary embeddings in torsion-free hyperbolic groups. Ann. Sci. Éc. Norm. Supér. (4) 44 (2011), no. 4, 631-681. Zbl 1245.20052 MR 2919979

[56] M. R. Pettet, Virtually free groups with finitely many outer automorphisms. Trans. Amer. Math. Soc. 349 (1997), no. 11, 4565-4587. Zbl 0891.20026 MR 1370649

[57] D. J. S. Robinson, A course in the theory of groups. $2^{\text {nd }}$ ed. Graduate Texts in Mathematics, 80. Springer, New York, 1996. Zbl 0836.20001 MR 1357169

[58] Z. Sela, The Nielsen-Thurston classification and automorphisms of a free group. I. Duke Math. J. 84 (1996), no. 2, 379-397. Zbl 0858.20019 MR 1404334

[59] Z. Sela, Structure and rigidity in (Gromov) hyperbolic groups and discrete groups in rank 1 Lie groups. II. Geom. Funct. Anal. 7 (1997), no. 3, 561-593. Zbl 0884.20025 MR 1466338 
[60] Z. Sela, Endomorphisms of hyperbolic groups. I. The Hopf property. Topology 38 (1999), no. 2, 301-321. Zbl 0929.20033 MR 1660337

[61] Z. Sela, Diophantine geometry over groups. I. Makanin-Razborov diagrams. Publ. Math. Inst. Hautes Études Sci. 93 (2001), 31-105. Zbl 1018.20034 MR 1863735

[62] J. Shor, A Scott conjecture for hyperbolic groups. Preprint 1999. http://www.math.unicaen.fr/ levitt/shorscott.pdf

[63] N. Touikan, Finding tracks in 2-complexes. Preprint 2009. arXiv:0906.3902v2 [math.GR]

Received February 6, 2013

V. Guirardel, Institut de Recherche Mathématique de Rennes,

Université de Rennes 1 et CNRS (UMR 6625), 263 avenue du Général Leclerc,

CS 74205, 35042 Rennes Cedex, France

e-mail: vincent.guirardel@univ-rennes1.fr

Gilbert Levitt, Laboratoire de Mathématiques Nicolas Oresme,

Université de Caen et CNRS (UMR 6139), BP 5186, CS 14032, 14032 Caen Cedex 5,

France

e-mail: levitt@unicaen.fr 